\title{
Lagrangian Floer theory on compact toric manifolds: survey
}

\author{
Kenji Fukaya, Yong-Geun Oh, Hiroshi Ohta, Kaoru Ono
}

ABSTRACT. This article is a survey of the Lagrangian Floer theory of toric manifolds, which summarizes the results obtained in a series of the present authors' papers [FOOO3, FOOO4, FOOO5]. In this survey, we discuss calculations of the Floer cohomology of Lagrangian $T^{n}$ orbits in compact toric manifolds. Applications to symplectic topology and to mirror symmetry are also discussed.

\section{Contents}

1. Introduction 230

2. Preliminary 232

2.1. Notations and terminologies 232

2.2. Moduli spaces of pseudo-holomorphic disks 234

3. A quick review of Lagrangian Floer theory 235

4. A quick review of toric manifold 238

5. Floer cohomology and potential function of the $T^{n}$ orbits 241

6. Examples $1 \quad 247$

7. Open-closed Gromov-Witten theory and operators $\mathfrak{q} \quad 249$

8. Floer cohomology with bulk deformation in the toric case 251

9. Leading term equation 257

10. Examples 2 260

11. Quantum cohomology and Jacobian ring 263

11.1. Jacobian ring over Novikov ring 263

11.2. Big quantum cohomology: a quick review 264

11.3. The isomorphism 'Jacobian ring = quantum cohomology' and its applications 265

Key words and phrases. Floer cohomology, mirror symmetry, toric manifolds, openclosed Gromov-Witten invariant, Saito theory, Landau-Ginzburg model, weakly unobstructed Lagrangian submanifolds, potential function, Jacobian ring, Frobenius structure.

(C)2012 International Press 
11.4. Construction of the homomorphism $\mathfrak{k}_{\mathfrak{b}}$

11.5. The homomorphism $\mathfrak{k}_{\mathfrak{s}_{\mathfrak{b}}}$ is an isomorphism 270

12. Poincaré duality and Residue pairing 275

12.1. Big quantum cohomology and Frobenius manifold $\quad 275$

12.2. A fragment of K. Saito theory. 278

12.3. Residue pairing on $\operatorname{Jac}\left(\mathfrak{P O}^{\mathfrak{b}}\right) \quad 280$

12.4. Residue pairing is Poincaré duality. 284

12.5. Operator $\mathfrak{p}$ and the Poincaré dual to $\mathfrak{k}_{\mathfrak{b}}$. 286

12.6. Annulus argument. 290

13. Examples $3 \quad 292$

Acknowledgements 295

$\begin{array}{ll}\text { References } & 295\end{array}$

\section{Introduction}

This is the survey of a series of present authors' papers [FOOO3, FOOO4, FOOO5] on the Lagrangian Floer theory of toric manifolds and its applications to mirror symmetry. The main purposes of the present survey are to convey systematic means of computations of Lagrangian Floer cohomology of the $T^{n}$-orbits in toric manifolds, to demonstrate various operations introduced in [FOOO1] Section 3.8 and apply them to the mirror symmetry between toric A model and Landau-Ginzburg B model and to symplectic topology of toric manifolds.

Let $X$ be a compact toric manifold with complex dimension $n$ and let $L(\mathbf{u})$ be a $T^{n}$ orbit. (Here $\mathbf{u}$ is an element of the interior of the moment polytope which parametrizes the location of the corresponding $T^{n}$ orbit. See Section 4 Formula (12).) We show that the cardinality (counted with multiplicity) of the pairs $(L(\mathbf{u}), b)$, for which Floer cohomology $H F((L(\mathbf{u}), b)$, $(L(\mathbf{u}), b) ; \Lambda)$ is nontrivial, is equal to the Betti number of $X$. (Theorem 11.9.) Here $b$ is an element of $H^{1}\left(L(\mathbf{u}) ; \Lambda_{0}\right) / H^{1}(L(\mathbf{u}) ; 2 \pi \sqrt{-1} \mathbb{Z})$ which deforms the Floer (pre-)coboundary map and so does the Floer cohomology of $L$.

The set of such pairs $(L(\mathbf{u}), b)$ one-one corresponds to the set of the critical point of a certain function $\mathfrak{P O}$, called the potential function. Then $\mathbf{u}$, the location of $L(\mathbf{u})$, is given by the valuation of the coordinates of the corresponding critical point. For the given toric manifold $X$, valuations of the critical points of $\mathfrak{P O}$ can be obtained by solving a system of explicitly calculable algebraic equations in a finite number of times. We illustrate these examples in sections 6 and 10. (We use the result of Cho-Oh $[\mathbf{C O}]$ for this calculation.)

The above mentioned one-one correspondence is induced by an isomorphism between quantum cohomology $Q H\left(X ; \Lambda_{0}\right)$ of $X$ and the Jacobian ring $\operatorname{Jac}(\mathfrak{P O})$ of the potential function $\mathfrak{P O}$. The origin of such a correspondence goes back to Givental $[\mathbf{G i 1}, \mathbf{G i 2}]$ and Batyrev $[\mathbf{B 1}, \mathbf{B 2}]$ in the case when 
$X$ is Fano. We remark that the rank of $Q H\left(X ; \Lambda_{0}\right)$ is the same as the Betti number of $X$, and the rank of $\operatorname{Jac}(\mathfrak{P O})$ is the same as the number of critical points of $\mathfrak{P O}$ counted with multiplicity.

The isomorphism $Q H\left(X ; \Lambda_{0}\right) \cong \operatorname{Jac}(\mathfrak{P O})$ is a ring isomorphism. In case $Q H(X ; \Lambda)$ is semi-simple, the $\operatorname{ring} Q H(X ; \Lambda)$ splits into the product of copies of the field $\Lambda$ and each factor of the product corresponds to a critical point of $\mathfrak{P O}$. (Proposition 11.8.)

In this way we associate a non-displaceable Lagrangian submanifold $L(\mathbf{u})$ to each of the direct factors of $Q H(X ; \Lambda)$. Entov-Polterovich $[\mathbf{E}, \mathbf{E P 1}$, EP2, EP3] and others [Os, Us] associated a Calabi quasi-homomorphism to each direct factor of $Q H(X ; \Lambda)$, and also associates non-displaceable Lagrangian submanifolds $L(\mathbf{u})$ to such Calabi quasi-homomorphisms. The non-displaceable Lagrangian submanifold associated by the EntovPolterovich's theory coincides with the one associated by the Lagrangian Floer theory, as we prove in [FOOO7]. (Our construction and proof are very different from Entov-Polterovich's, though.)

The ring isomorphism $Q H\left(X ; \Lambda_{0}\right) \cong \operatorname{Jac}(\mathfrak{P O})$ is generalized to the context of big quantum cohomology in the left hand side and the potential function of the Lagrangian Floer theory with bulk deformations in the right hand side. We call the corresponding potential function the potential function with bulk.

Furthermore this ring isomorphism intertwines the pairings, which are the Poincaré duality pairing in the left hand side and (a version of) the residue pairing in the right hand side. This gives rise to an isomorphism of the relevant Frobenius manifold structures present in the two sides: One is the Frobenius manifold structure induced by big quantum cohomology, which is due to Dubrovin [Dub], and the other is the one associated to the isolated singularity by Saito [Sa, MSa]. This isomorphism can be regarded as a version of mirror symmetry between Toric A model and LandauGinzburg B model. It is closely related to the story of Hori-Vafa $[\mathbf{H V}]$ and also of Givental.

The mirror symmetry between the toric manifold and the singularity theory have been studied by many mathematicians. Besides those already mentioned above, we provide a list of some of them, which is not exhaustive by any means.

In this survey, we focus on the case in which we study the $A$ model (symplectic geometry and pseudo-holomorphic curve) on the toric manifold side and the $B$ model (deformation theory and complex geometry) on singularity theory side. The papers [Aur1, Aur2, Bar, CLe, CO, Gro1, Gro2, GPS, Iri1, Iri2, Iri3, OT, Ta, W] also deal with that case.

The other side of the story, namely $B$ model in toric side and $A$ model in singularity theory side, has been more extensively studied than the side of present survey. The papers $[\mathbf{A b 1}, \mathbf{A K O}, \mathbf{F L T Z}, \mathbf{S e 2}, \mathbf{U e}, \mathbf{U Y}]$ deal with this side. 


\section{Preliminary}

2.1. Notations and terminologies. The universal Novikov ring $\Lambda_{0}$ is the set of all formal sums

$$
\sum_{i=0}^{\infty} a_{i} T^{\lambda_{i}}
$$

where $a_{i} \in \mathbb{C}$ and $\lambda_{i} \in \mathbb{R}_{\geq 0}$ such that $\lim _{i \rightarrow \infty} \lambda_{i}=\infty$, and $T$ is a formal parameter. We allow $\lambda_{i} \in \mathbb{R}$ in (1) (namely negative $\lambda_{i}$ ) to define $\Lambda$ which we call universal Novikov field. It is a field of fraction of $\Lambda_{0}$. We require $\lambda_{i}>0$ in (1) to define $\Lambda_{+}$, which is the maximal ideal of $\Lambda_{0}$.

We define a valuation $\mathfrak{v}_{T}$ on $\Lambda$ by

$$
\mathfrak{v}_{T}\left(\sum_{i=0}^{\infty} a_{i} T^{\lambda_{i}}\right)=\inf \left\{\lambda_{i} \mid a_{i} \neq 0\right\} .
$$

(Here we assume $\lambda_{i} \neq \lambda_{j}$ for $i \neq j$.) $\Lambda, \Lambda_{0}, \Lambda_{+}$are complete with respect to $\mathfrak{v}_{T}$ and $\left(\Lambda_{0}, \Lambda_{+}\right)$is a valuational ring with valuation $\mathfrak{v}_{T}$.

REMARK 2.1. In [FOOO1] a slightly different Novikov ring $\Lambda_{0, \text { nov }}$ which contains another formal parameter $e$ is used. The role of $e$ is to adjust all the operators appearing in the story so that they have well-defined degree. (e has degree 2.) In [FOOO3, FOOO4, FOOO5] and this paper we use $\Lambda_{0}$ since ring theoretical properties of $\Lambda_{0}$ is better than one of $\Lambda_{0, \text { nov }}$. As a drawback only the parities of various operators are well-defined.

Let $Z_{1}, \ldots, Z_{m}$ be variables. We define the strictly convergent power series ring

$$
\Lambda_{0}\left\langle\left\langle Z_{1}, \ldots, Z_{m}\right\rangle\right\rangle
$$

as the set of all formal sums

$$
\sum_{k_{1}=0}^{\infty} \ldots \sum_{k_{m}=0}^{\infty} C_{k_{1} \ldots k_{m}} Z_{1}^{k_{1}} \ldots Z_{m}^{k_{m}}
$$

where $C_{k_{1} \ldots k_{m}} \in \Lambda_{0}$ such that

$$
\lim _{k_{1}+\cdots+k_{m} \rightarrow \infty} \mathfrak{v}_{T}\left(C_{k_{1} \ldots k_{m}}\right)=+\infty .
$$

We define strictly convergent Laurent power series ring

$$
\Lambda_{0}\left\langle\left\langle Z_{1}, Z_{1}^{-1}, \ldots, Z_{m}, Z_{m}^{-1}\right\rangle\right\rangle
$$

as the set of all formal sums

$$
\sum_{k_{1} \in \mathbb{Z}} \cdots \sum_{k_{m} \in \mathbb{Z}} C_{k_{1} \ldots k_{m}} Z_{1}^{k_{1}} \ldots Z_{m}^{k_{m}}
$$

where $C_{k_{1} \ldots k_{m}} \in \Lambda_{0}$ such that

$$
\lim _{\left|k_{1}\right|+\cdots+\left|k_{m}\right| \rightarrow \infty} \mathfrak{v}_{T}\left(C_{k_{1} \ldots k_{m}}\right)=+\infty .
$$


See $[$ BGR $]$ about those rings.

We also define

$$
\Lambda\left\langle\left\langle Z_{1}, Z_{1}^{-1}, \ldots, Z_{m}, Z_{m}^{-1}\right\rangle\right\rangle=\Lambda_{0}\left\langle\left\langle Z_{1}, Z_{1}^{-1}, \ldots, Z_{m}, Z_{m}^{-1}\right\rangle\right\rangle \otimes_{\Lambda_{0}} \Lambda .
$$

The definition of $\Lambda\left\langle\left\langle Z_{1}, \ldots, Z_{m}\right\rangle\right\rangle$ is similar.

Let $C$ be a graded free $\Lambda_{0}$ module. The valuation $\mathfrak{v}_{T}$ induces a norm on $C$ in an obvious way, by which $C$ is complete. We define its degree shift $C[1]$ by $C[1]^{k}=C^{k+1}$. The shifted degree $\operatorname{deg}^{\prime}$ is defined by

$$
\operatorname{deg}^{\prime} x=\operatorname{deg} x-1 \text {. }
$$

We put

$$
B_{k} C=\underbrace{C \otimes \cdots \otimes C}_{k \text { times }} .
$$

Let $\widehat{B} C=\widehat{\bigoplus}_{k=0}^{\infty} B_{k} C$ be the completed direct sum of them. Let $\mathfrak{S}_{k}$ be the symmetric group of order $k$ !. It acts on $B_{k} C$ by

$$
\sigma \cdot\left(x_{1} \otimes \cdots \otimes x_{k}\right)=(-1)^{*} x_{\sigma(1)} \otimes \cdots \otimes x_{\sigma(k)}
$$

where $*=\sum_{i<j: \sigma(i)>\sigma(j)} \operatorname{deg} x_{i} \operatorname{deg} x_{j}$. We define $E_{k} C$ as the subset of $\mathfrak{S}_{k}$ invariant elements of $B_{k} C$ and put $\widehat{E} C=\widehat{\bigoplus}_{k=0}^{\infty} E_{k} C$ its completed direct sum.

On $B C$ we define a coalgebra structure $\Delta: B C \rightarrow(B C)^{\otimes 2}$ by

$$
\Delta\left(x_{1} \otimes \cdots \otimes x_{k}\right)=\sum_{i=0}^{k}\left(x_{1} \otimes \cdots \otimes x_{i}\right) \otimes\left(x_{i+1} \otimes \cdots \otimes x_{k}\right) .
$$

(Note the summand in the case $i=0$ is $\left.1 \otimes\left(x_{1} \otimes \cdots \otimes x_{k}\right).\right) \Delta$ is coassociative.

We can define $\Delta: E C \rightarrow(E C)^{\otimes 2}$ by restriction. It is coassociative and graded cocommutative.

We also consider a map $\Delta^{k-1}: B C \rightarrow(B C)^{\otimes k}$

$$
\Delta^{k-1}=(\Delta \otimes \underbrace{i d \otimes \cdots \otimes i d}_{k-2}) \circ(\Delta \otimes \underbrace{i d \otimes \cdots \otimes i d}_{k-3}) \circ \cdots \circ \Delta .
$$

For an indecomposable element $\mathbf{x} \in B C$, it can be expressed as

$$
\Delta^{k-1}(\mathbf{x})=\sum_{c} \mathbf{x}_{c}^{k ; 1} \otimes \cdots \otimes \mathbf{x}_{c}^{k ; k}
$$

where $c$ runs over some index set. We use the same notation for $E C$. 
2.2. Moduli spaces of pseudo-holomorphic disks. Lagrangian Floer theory is based on the moduli space of pseudo-holomorphic disks. We recall its definition below. See [FOOO1] subsection 2.1.2 for detail.

Let $X=(X, \omega)$ be a symplectic manifold and $L$ its Lagrangian submanifold. We pick a compatible almost complex structure $J$ on $X$. Let $\beta \in H_{2}(X, L ; \mathbb{Z})$.

The moduli space $\mathcal{M}_{k+1 ; \ell}^{\text {main }}(\beta)$ is the compactified moduli space of the genus zero bordered holomorphic maps $u:(\Sigma, \partial \Sigma) \rightarrow(X, L)$, in class $\beta \in$ $H_{2}(X, L(u) ; \mathbb{Z})$ with $k+1$ boundary marked points and $\ell$ interior marked points. This means the following:

Conditions 2.2. 1 1) $\Sigma$ is a connected union of disks and spheres, which we call (irreducible) components. We assume the intersection of two different irreducible components is either one point or empty. The intersection of two disk components is if nonempty, a boundary point of both of the components. The intersection of a disk and a sphere component is an interior point of the disk component. We assume that intersection of three different components is empty. We also require $\Sigma$ to be simply connected. A point which belongs to two different components is called a singular point.

2) $u: \Sigma \rightarrow X$ is a continuous map which is $J$-holomorphic on each of the components. $u(\partial \Sigma) \subset L$. Here $\partial \Sigma$ is the union of the boundary of disk components.

3) There are $k+1$ points $z_{0}, \ldots, z_{k}$ on $\partial \Sigma$. (We call them boundary marked points.) They are mutually distinct. None of them are singular point. We require the order of $k+1$ boundary marked points to respect the counter-clockwise cyclic order of the boundary of $\Sigma$.

4) There are $\ell$ points $z_{1}^{+}, \ldots, z_{\ell}^{+}$on $\Sigma \backslash \partial \Sigma$. (We call them interior marked points.) They are mutually distinct. None of them are singular point.

5) For each of the components $\Sigma_{a}$ of $\Sigma$, one of the following conditions hold:

a) $u$ is not a constant map on $\Sigma_{a}$.

b) $\Sigma_{a}$ is a disk component. We have $2 n_{\text {int }}+n_{\text {bdry }} \geq 3$. Here $n_{\text {int }}$ is the sum of the numbers of the interior marked points and the interior singular points. $n_{\text {bdry }}$ is the sum of the numbers of the boundary marked points and the boundary singular points.

c) $\Sigma_{a}$ is a sphere component. The sum of the numbers of the marked points and the singular points on $\Sigma_{a}$ is $\geq 3$.

The condition 5) is called the stability condition. It is equivalent to the condition that the automorphism group of this element is a finite group.

In case $\ell=0$ we write $\mathcal{M}_{k+1}^{\text {main }}(\beta)$ in place of $\mathcal{M}_{k+1 ; 0}^{\text {main }}(\beta)$.

We define the evaluation maps

$$
\mathrm{ev}: \mathcal{M}_{k+1 ; \ell}^{\operatorname{main}}(\beta) \rightarrow X^{\ell} \times L^{k+1}
$$


where we put

$$
\mathrm{ev}=\left(\mathrm{ev}^{+}, \mathrm{ev}\right)=\left(\mathrm{ev}_{1}^{+}, \ldots, \mathrm{ev}_{\ell}^{+} ; \mathrm{ev}_{0}, \ldots, \mathrm{ev}_{k}\right),
$$

as follows:

$$
\mathrm{ev}_{i}(\Sigma, u)=u\left(z_{i}\right)
$$

where $z_{i}$ is the $i$-th boundary marked point as in 3 ).

$$
\mathrm{ev}_{i}^{+}(\Sigma, u)=u\left(z_{i}^{+}\right)
$$

where $z_{i}^{+}$is the interior marked point as in 4).

Our moduli spaces $\mathcal{M}_{k+1 ; \ell}^{\text {main }}(\beta)$ have Kuranishi structure in the sense of [FO] section 5 and [FOOO1] section A1.

Its boundary is described by using fiber product. For example, in case $\ell=0$ we have the equality

$$
\partial \mathcal{M}_{k+1}^{\text {main }}(\beta)=\bigcup_{k_{1}+k_{2}=k+1} \bigcup_{\beta_{1}+\beta_{2}=\beta} \bigcup_{i=1}^{k_{2}} \mathcal{M}_{k_{1}+1}^{\text {main }}\left(\beta_{1}\right) \text { ev }_{0} \times_{\mathrm{ev}_{i}} \mathcal{M}_{k_{2}+1}^{\text {main }}\left(\beta_{2}\right) .
$$

as spaces with Kuranishi structures. ([FOOO1] subsection 7.1.1.)

\section{A quick review of Lagrangian Floer theory}

Let $X=(X, \omega)$ be a symplectic manifold and $L$ its Lagrangian submanifold. We assume $L$ is oriented and spin. (Actually relative spinness in the sense of [FOOO1] Definition 1.6 is enough.)

In [FOOO1] Theorem A, we defined a structure of gapped unital filtered $A_{\infty}$ algebra $\left\{\mathfrak{m}_{k} \mid k=0,1, \ldots\right\}$ on the cohomology group $H\left(L ; \Lambda_{0}\right)$ of $L$ with $\Lambda_{0}$ coefficient.

Namely there exists a sequence of operators

$$
\mathfrak{m}_{k}: B_{k} H\left(L ; \Lambda_{0}\right)[1] \rightarrow H\left(L ; \Lambda_{0}\right)[1]
$$

of odd degree ${ }^{1}$ (for $k \geq 0$ ).

THEOREM 3.1. 1)

$$
\begin{aligned}
& \sum_{k_{1}+k_{2}=k+1} \sum_{i=1}^{k_{2}}(-1)^{*} \mathfrak{m}_{k_{2}}\left(x_{1}, \ldots, \mathfrak{m}_{k_{1}}\left(x_{i}, \ldots, x_{i+k_{1}-1}\right), \ldots, x_{k}\right)=0 \\
& \text { where } *=\operatorname{deg}^{\prime} x_{1}+\cdots+\operatorname{deg}^{\prime} x_{i-1} \\
& \text { 2) } \mathfrak{m}_{0}(1) \equiv 0 \bmod \Lambda_{+}
\end{aligned}
$$

\footnotetext{
${ }^{1}$ See Remark 2.1. Only the parity of the degree is well-defined in Floer cohomology over $\Lambda_{0}$.
} 
3) (Unitality) $\mathbf{e}=\mathrm{PD}[L] \in H^{0}\left(L ; \Lambda_{0}\right)$ is the strict unit. (Here $\mathrm{PD}$ : $H_{k}(L) \rightarrow H^{n-k}(L)$ is the Poincaré duality.) Namely

$$
\mathfrak{m}_{k+1}\left(x_{1}, \ldots, \mathbf{e}, \ldots, x_{k}\right)=0 \quad \text { for } k \geq 2 \text { or } k=0 .
$$

and

$$
\mathfrak{m}_{2}(\mathbf{e}, x)=(-1)^{\operatorname{deg} x} \mathfrak{m}_{2}(x, \mathbf{e})=x .
$$

4) (G-gappedness) There exists an additive discrete submonoid $G=$ $\left\{\lambda_{i} \mid i=0,1,2, \ldots\right\} \quad\left(\lambda_{0}=0<\lambda_{1}<\lambda_{2}<\cdots, \lim _{i \rightarrow \infty} \lambda_{i}=\infty\right) \quad$ of $\mathbb{R}_{\geq 0}$ such that our structure is G-gapped. Namely $\mathfrak{m}_{k}$ is written as

$$
\mathfrak{m}_{k}=\sum_{i=0}^{\infty} T^{\lambda_{i}} \mathfrak{m}_{k, i}
$$

where $\mathfrak{m}_{k, i}: B_{k} H(L ; \mathbb{C})[1] \rightarrow H(L ; \mathbb{C})[1]$ is $\mathbb{C}$-linear.

5) $\mathfrak{m}_{2,0}$ coincides with cup product up to sign.

The triple $\left(C,\left\{\mathfrak{m}_{k}\right\}, \mathbf{e}\right)$ that satisfies 1$)-4$ ) of Theorem 3.1 (with $H\left(L ; \Lambda_{0}\right)$ being replaced by $C$ ) is called a $G$-gapped unital filtered $A_{\infty}$ algebra.

The operator $\mathfrak{m}_{k}$ is constructed by using the moduli spaces $\mathcal{M}_{k+1}^{\text {main }}(\beta)$ as follows. (Here we use de Rham cohomology, following [FOOO3, FOOO4, FOOO5, Fu2, Fu3]. In [FOOO1] singular homology is used. Morse homology version is in [FOOO2].)

Let $h_{1}, \ldots, h_{k}$ be differential forms on $L$. We define a differential form $\mathfrak{m}_{k, \beta}\left(h_{1}, \ldots, h_{k}\right)$ on $L$ as follows:

$$
\mathfrak{m}_{k, \beta}\left(h_{1}, \ldots, h_{k}\right)=\mathrm{ev}_{0 !}\left(\mathrm{ev}_{1}, \ldots, \mathrm{ev}_{k}\right)^{*}\left(h_{1} \times \cdots \times h_{k}\right)
$$

for $(k, \beta) \neq(1,0)$. We use evaluation maps (7) in (10). We put

$$
\mathfrak{m}_{1,0}(h)=(-1)^{n+\operatorname{deg} h+1} d h,
$$

where $d$ is the de Rham differential. (See [FOOO1] Remark 3.5.8.)

Here we regard $h_{1} \times \cdots \times h_{k}$ as a differential form on $L^{k}$. Then the pull back $\left(\mathrm{ev}_{1}, \ldots, \mathrm{ev}_{k}\right)^{*}$ defines a differential form on $\mathcal{M}_{k+1}^{\text {main }}(\beta)$. The symbol $\mathrm{ev}_{0}$ ! denotes the integration along the fiber associated to the map $\mathrm{ev}_{0}$ : $\mathcal{M}_{k+1}^{\text {main }}(\beta) \rightarrow L$. We remark that $\mathcal{M}_{k+1}^{\text {main }}(\beta)$ itself is not necessarily transversal. So it may have wrong dimension. However we can use general theory of Kuranishi structure to obtain a multisection $\mathfrak{s}([\mathbf{F O}]$ section 5 , [FOOO1] section A1) so that the perturbed moduli space $\mathcal{M}_{k+1}^{\operatorname{main}}(\beta)^{\mathfrak{s}}$ (that is the zero point set of the multisection $\mathfrak{s}$ ) has a virtual fundamental chain (over $\mathbb{Q}$ ). However still after perturbation, the map ev $:: \mathcal{M}_{k+1}^{\text {main }}(\beta)^{\mathfrak{s}} \rightarrow L$ may not be a submersion on the perturbed moduli space $\mathcal{M}_{k+1}^{\operatorname{main}}(\beta)^{\mathfrak{s}}$. So we take a continuous family of perturbations written as $\left\{\mathfrak{s}_{w}\right\}_{w \in W}$ parametrized by a certain smooth manifold $W$ so that

$$
\mathrm{ev}_{0}^{W}: \bigcup_{w \in W}\left(\mathcal{M}_{k+1}^{\operatorname{main}}(\beta)^{\mathfrak{s}_{w}} \times\{w\}\right) \rightarrow L
$$


is a submersion. ${ }^{2}$ So we can justify $(10)$ as

$$
\mathfrak{m}_{k, \beta}\left(h_{1}, \ldots, h_{k}\right)=\mathrm{ev}_{0 !}^{W}\left(\left(\mathrm{ev}_{1}, \ldots, \mathrm{ev}_{k}\right)^{*}\left(h_{1} \times \cdots \times h_{k}\right) \wedge \omega_{W}\right) .
$$

Here $\omega_{W}$ is a smooth form of degree $\operatorname{dim} W$ on $W$ that has compact support and satisfies $\int_{W} \omega_{W}=1$. We pull it back to $\bigcup_{w \in W}\left(\mathcal{M}_{k+1}^{\operatorname{main}}(\beta)^{\mathfrak{s}_{w}} \times\{w\}\right)$ in an obvious way. The fiberwise evaluation map $\operatorname{ev}_{0}^{W}$ is $\operatorname{ev}_{0}$ on $\mathcal{M}_{k+1}^{\operatorname{main}}(\beta)^{\mathfrak{s} w} \times\{w\}$.

We omit the detail of this construction and refer [FOOO4] section 12 or [Fu2] section 13. In the toric case, which is the case of our main interest in this article, this construction can be simplified in most of the cases. Namely $\mathrm{ev}_{0}: \mathcal{M}_{k+1}^{\text {main }}(\beta)^{\mathfrak{s}} \rightarrow L$ itself can be taken to be a submersion (without using continuous family). See Section 5 .

We now put

$$
\mathfrak{m}_{k}=\sum_{\beta \in H_{2}(X, L ; \mathbb{Z})} T^{(\beta \cap[\omega]) / 2 \pi} \mathfrak{m}_{k, \beta}
$$

We can use various properties of the moduli space to check Theorem 3.1. In fact, for example, Theorem 3.11 ) is a consequece of Formula (8) and Theorem 3.14 ) is a consequence of Gromov compactness.

Thus we obtain a structure of $G$-gapped unital filtered $A_{\infty}$ algebra on de Rham complex of $L$. Then it induces one on cohomology $H\left(X, L ; \Lambda_{0}\right)$, by a purely algebraic result. ([FOOO1] Theorem 5.4.2.)

The filtered $A_{\infty}$ algebra $\left(H\left(X, L ; \Lambda_{0}\right),\left\{\mathfrak{m}_{k} \mid k=0,1, \ldots\right\}\right)$ is independent of the choices (such as compatible almost complex structures and perturbations etc.) up to an isomorphism of a gapped unital filtered $A_{\infty}$ algebra, (that is gapped unital filtered $A_{\infty}$ homomorphism which has an inverse). We omit the precise definition of this notion and refer readers to [FOOO1] Definition 3.2.29 and Proposition 5.4.5.

Let $\left(C,\left\{\mathfrak{m}_{k} \mid k=0,1, \ldots\right\}, \mathbf{e}\right)$ be a unital filtered $A_{\infty}$ algebra. We define its weak Maurer-Cartan scheme $\widehat{\mathcal{M}}_{\text {weak }}(C)$ as the set of solutions of the equation

$$
\sum_{k=0}^{\infty} \mathfrak{m}_{k}(b, \ldots, b) \equiv 0 \quad \bmod \Lambda_{0} \mathbf{e}
$$

for $b \in C^{\text {odd }}$, with $b \equiv 0 \bmod \Lambda_{+}$. (Here and hereafter e denotes the unit.)

For $b \in C^{\text {odd }}$, with $b \equiv 0 \bmod \Lambda_{+}$, we define $\mathfrak{m}_{k}^{b}$ by

$$
\mathfrak{m}_{k}^{b}\left(x_{1}, \ldots, x_{k}\right)=\sum_{m_{0}=0}^{\infty} \ldots \sum_{m_{k}=0}^{\infty} \mathfrak{m}_{k}(\underbrace{b, \ldots, b}_{m_{0}}, x_{1}, \underbrace{b, \ldots, b}_{m_{1}}, \ldots, x_{k}, \underbrace{b, \ldots, b}_{m_{k}}) .
$$

The right hand side converges in $\mathfrak{v}_{T}$ topology. We can show that $\left(C,\left\{\mathfrak{m}_{k}^{b} \mid\right.\right.$ $k=0,1, \ldots\}, \mathbf{e})$ is a filtered $A_{\infty}$ algebra.

In our geometric situation, where $C=H\left(L ; \Lambda_{0}\right)$, we can remove the assumption $b \equiv 0 \bmod \Lambda_{+}$using a trick due to Cho [Cho3] and can define

\footnotetext{
${ }^{2}$ Actually the parameter space $W$ is defined only locally. See [FOOO4] section 12 .
} 
$\mathfrak{m}_{k}^{b}$ for any $b \in H^{o d d}\left(L ; \Lambda_{0}\right)$. (See [FOOO3] section 12 for toric case and [Fu3] section 5 for the general case.) Moreover the left hand side of (11) makes sense for any $b \in H^{o d d}\left(L ; \Lambda_{0}\right)$. In case we need to distinguish it from the case $b \in H^{o d d}\left(L ; \Lambda_{+}\right)$, we denote the former by $\widehat{\mathcal{M}}_{\text {weak }}\left(H\left(L ; \Lambda_{0}\right) ; \Lambda_{0}\right)$.

It is easy to see that $\mathfrak{m}_{0}^{b}(1)$ coincides with the left hand side of (11). Therefore if $b \in \widehat{\mathcal{M}}_{\text {weak }}(C)$ then $\mathfrak{m}_{0}^{b}(1)=c \mathbf{e}$ for some $c \in \Lambda_{+}$. It follows that

$$
\left(\mathfrak{m}_{1}^{b} \circ \mathfrak{m}_{1}^{b}\right)(x)=-c\left(\mathfrak{m}_{2}^{b}(\mathbf{e}, x)+(-1)^{\operatorname{deg}^{\prime} x} \mathfrak{m}_{2}^{b}(x, \mathbf{e})\right)=0 .
$$

Here we use Theorem 3.1 1) in the first equality and Theorem 3.13 ) in the second equality. Now we define

Definition 3.2. Let $b \in H^{o d d}\left(L ; \Lambda_{0}\right)$. We define Floer cohomology by:

$$
H F\left((L, b),(L, b) ; \Lambda_{0}\right)=\frac{\operatorname{Ker}\left(\mathfrak{m}_{1}^{b}\right)}{\operatorname{Im}\left(\mathfrak{m}_{1}^{b}\right)} .
$$

$H F((L, b),(L, b) ; \Lambda)$ is defined by taking $\otimes_{\Lambda_{0}} \Lambda$.

It is proved in [FOOO1] Proposition 3.7.75 and the discussion right after that (general case, singular homology version) [FOOO4] section 8 (toric case, de Rham homology version) that $\operatorname{HF}((L, b),(L, b) ; \Lambda) \neq 0$ implies that $L$ is Hamiltonian non-displaceable. ${ }^{3}$ Namely for any Hamiltonian diffeomorphism $F: X \rightarrow X$ we have $F(L) \cap L \neq \emptyset$.

Let $b \in \widehat{\mathcal{M}}_{\text {weak }}(C)$. Then there exists $\mathfrak{P O}(b) \in \Lambda_{+}$such that

$$
\sum_{k=0}^{\infty} \mathfrak{m}_{k}(b, \ldots, b)=\mathfrak{P O}(b) \mathbf{e} .
$$

Definition 3.3. We call $\mathfrak{P O}: \widehat{\mathcal{M}}_{\text {weak }}(C) \rightarrow \Lambda_{+}$, the potential function.

In the geometric situation we have $\mathfrak{P O}: \widehat{\mathcal{M}}_{\text {weak }}\left(H\left(L ; \Lambda_{0}\right) ; \Lambda_{0}\right) \rightarrow \Lambda_{+}$.

\section{A quick review of toric manifold}

In this section we review a very small portion of the theory of toric variety. We explain only the points we use in this article. See for example $[$ Ful $]$ for an account of toric variety.

Let $(X, \omega, J)$ be a Kähler manifold, where $J$ is its complex structure and $\omega$ is its Kähler form. Let $n$ be the complex dimension of $X$. We assume $n$ dimensional real torus $T^{n}=\left(S^{1}\right)^{n}$ acts effectively on $X$ such that $J$ and $\omega$ are preserved by the action. We call such $(X, \omega, J)$ a Kähler toric manifold if the $T^{n}$ action has a moment map in the sense we describe below. Hereafter we simply say $(X, \omega, J)$ (or $X)$ is a toric manifold.

\footnotetext{
${ }^{3}$ We need to take $\Lambda$ (not $\Lambda_{0}$ ) for the coefficient ring for this statement. Actually $H F\left((L, b),(L, b) ; \Lambda_{0}\right)=0$ never occurs when Floer cohomology is defined.
} 
Let $(X, \omega, J)$ be as above. We say a map $\pi=\left(\pi_{1}, \ldots, \pi_{n}\right): X \rightarrow \mathbb{R}^{n}$ is a moment map if the following holds. We consider the $i$-th factor $S_{i}^{1}$ of $T^{n}$. (Here $i=1, \ldots, n$.) Then $\pi_{i}: X \rightarrow \mathbb{R}$ is the moment map of the action of $S_{i}^{1}$. In other words, we have the following identity of $\pi_{i}$

$$
d \pi_{i}(X)=\omega(X, \tilde{\mathfrak{t}}),
$$

where $\tilde{\mathfrak{t}}$ is the Killing vector field associated to the action of the circle $S_{i}^{1}$ on $X$.

Let $\mathbf{u} \in \operatorname{Int} P$. Then the inverse image $\pi^{-1}(\mathbf{u})$ is a Lagrangian submanifold which is an orbit of the $T^{n}$ action. We put

$$
L(\mathbf{u})=\pi^{-1}(\mathbf{u}) .
$$

This is a Lagrangian torus. The main purpose of this article is to study Lagrangian Floer cohomology for such $L(\mathbf{u})$.

It is well-known that $P=\pi(X)$ is a convex polytope. We can find a finitely many affine functions $\ell_{j}: \mathbb{R}^{n} \rightarrow \mathbb{R}(j=1, \ldots, m)$ such that

$$
P=\left\{\mathbf{u} \in \mathbb{R}^{n} \mid \ell_{j}(\mathbf{u}) \geq 0, \quad \forall j=1, \ldots, m\right\} .
$$

We put $\partial_{j} P=\left\{\mathbf{u} \in P \mid \ell_{j}(\mathbf{u})=0\right\}$ and $D_{j}=\pi^{-1}\left(\partial_{j} P\right) .\left(\operatorname{dim}_{\mathbb{R}} \partial_{j} P=n-1\right.$. $)$ $D_{1} \cup \cdots \cup D_{m}$ is called the toric divisor.

Moreover we may choose $\ell_{j}$ so that the following holds.

Conditions 4.1. 1) We put

$$
d \ell_{j}=\vec{v}_{j}=\left(v_{j, 1}, \ldots, v_{j, n}\right) \in \mathbb{R}^{n} .
$$

Then $v_{j, i} \in \mathbb{Z}$.

2) Let $p$ be a vertex of $P$. Then the number of faces $\partial_{j} P$ which contain $p$ is $n$. Let $\partial_{j_{1}} P, \ldots, \partial_{j_{n}} P$ be those faces. Then $\vec{v}_{j_{1}}, \ldots, \vec{v}_{j_{n}}$ (which is contained in $\mathbb{Z}^{n}$ by item 1)) is a basis of $\mathbb{Z}^{n}$.

The affine function $\ell_{j}$ has the following geometric interpretation. Let $\mathbf{u} \in \operatorname{Int} P$. There exists $m$ elements $\beta_{j} \in H_{2}(X, L(\mathbf{u}) ; \mathbb{Z})$ such that

$$
\beta_{j} \cap D_{j^{\prime}}= \begin{cases}1 & j=j^{\prime} \\ 0 & j \neq j^{\prime}\end{cases}
$$

Then we have

$$
2 \pi \ell_{j}(\mathbf{u})=\int_{\beta_{j}} \omega
$$

The existence of such $\ell_{j}$ and the property above is proved in $[\mathbf{G u}]$ Theorem 4.5. (See [FOOO3] section 2 also.) 
EXAMPLE 4.2. We consider the complex projective space $\mathbb{C} P^{n}$. Using homogeneous coordinate $\left[x_{0}: x_{1}: \cdots: x_{n}\right]$ we define $T^{n}$ action by

$$
\left(t_{1}, \ldots, t_{n}\right) \cdot\left[x_{0}: \cdots: x_{n}\right]=\left[x_{0}: e^{2 \pi \sqrt{-1} t_{1}} x_{1}: \cdots: e^{2 \pi \sqrt{-1} t_{n}} x_{n}\right] .
$$

(Here we identify $\mathbb{R} / \mathbb{Z} \cong S^{1}$.) The moment map $\pi=\left(\pi_{1}, \ldots, \pi_{n}\right)$ is given by

$$
\pi_{i}\left(\left[x_{0}: \cdots: x_{n}\right]\right)=\frac{\left|x_{i}\right|^{2}}{\left|x_{0}\right|^{2}+\cdots+\left|x_{n}\right|^{2}} .
$$

Its moment polytope $P_{0}$ is a simplex that is:

$$
P_{0}=\left\{\left(u_{1}, \ldots, u_{n}\right) \mid 0 \leq u_{i}, i=1, \ldots, n, \sum_{i=0}^{n} u_{i} \leq 1\right\} .
$$

We have

$$
\ell_{i}\left(u_{1}, \ldots, u_{n}\right)= \begin{cases}u_{i} & i \neq 0 \\ 1-\sum_{j=0}^{n} u_{j} & i=0\end{cases}
$$

EXAmple 4.3. We consider $\mathbb{C} P^{2}$ as above. For $1>\alpha>0$, let us consider

$$
P(\alpha)=P_{0} \backslash\left\{\left(u_{1}, u_{2}\right) \in P_{0} \mid u_{2}>1-\alpha\right\}=\left\{\left(u_{1}, u_{2}\right) \in P_{0} \mid u_{2} \leq 1-\alpha\right\} .
$$

The inverse image $\pi^{-1}\left(\left\{\left(u_{1}, u_{2}\right) \in P_{0} \mid u_{2}>1-\alpha\right\}\right)$ is a ball of radius $\sqrt{\alpha / 2}$ centered at $[0: 1: 0]$. The boundary of $\pi^{-1}(P(\alpha))$ has an induced contact form which is identified with the standard contact form of $S^{3}$. We identify two points on $\partial \pi^{-1}(P(\alpha))$ if they lie on the same orbit of Reeb flow. After this identification we obtain from $\pi^{-1}(P(\alpha))$ a symplectic manifold which we write $X(\alpha)=\mathbb{C} P^{2} \# \overline{\mathbb{C} P}^{2}(\alpha)$.

It is well-known (see for example [MS] section 6.2) and can be proved from the above description that $X(\alpha)$ is a blow up of $\mathbb{C} P^{2}$ with Kähler form $\omega$ such that the symplectic area of the exceptional divisor is $\alpha$.

The $T^{2}$ action on $\mathbb{C} P^{2}$ induces a $T^{2}$ action on $X(\alpha)$ so that it becomes a toric manifold. The moment polytope is $P(\alpha)$.

There are 4 faces of $P(\alpha)$ and 4 affine functions $\ell_{i}(i=0,1,2,3)$. Three of them are $\ell_{0}, \ell_{1}, \ell_{2}$ as in (16). The fourth one is given by

$$
\ell_{3}\left(u_{1}, u_{2}\right)=1-\alpha-u_{2} \text {. }
$$

EXAMPLE 4.4. We can blow up again and may regard a two points blow up of $\mathbb{C} P^{2}$ as a toric manifold. For $\alpha, \alpha^{\prime}>0$, with $\alpha+\alpha^{\prime}<1$ we consider the polytope

$$
P\left(\alpha, \alpha^{\prime}\right)=\left\{\left(u_{1}, u_{2}\right) \in P_{0} \mid u_{2} \leq 1-\alpha, u_{1}+u_{2} \geq \alpha^{\prime}\right\} .
$$

There exists a toric manifold $X\left(\alpha, \alpha^{\prime}\right)$ that is a two points blow up of $\mathbb{C} P^{2}$ and whose moment polytope is $P\left(\alpha, \alpha^{\prime}\right)$. 
$P\left(\alpha, \alpha^{\prime}\right)$ has 5 faces. There are 5 affine functions $\ell_{0}, \ldots, \ell_{4}$ associated to each of the faces. $\ell_{0}, \ell_{1}, \ell_{2}$ are as in (16) and $\ell_{3}$ is as in (17). $\ell_{4}$ is given by

$$
\ell_{4}\left(u_{1}, u_{2}\right)=u_{1}+u_{2}-\alpha^{\prime}
$$

\section{Floer cohomology and potential function of the $T^{n}$ orbits}

In this section we give a description of Floer cohomology of the $T^{n}$ orbit $L(\mathbf{u})$ of the toric manifold $X$. Here $\mathbf{u} \in \operatorname{Int} P$ and $P$ is the moment polytope of $X$.

In this toric case the calculation of the Floer cohomology becomes significantly simpler. This is because in this case the calculation of Floer cohomology is reduced to the calculation of the potential function. Moreover the leading order term of the potential function is calculated by the work of Cho-Oh $[\mathbf{C O}]$. We will explain those points in this section.

We first fix a basis of $H^{1}(L(\mathbf{u}) ; \mathbb{Z})$ as follows. In Section 4 we fix a split$\operatorname{ting} T^{n}=\left(S^{1}\right)^{n}$ and the associated coordinate $\left(t_{1}, \ldots, t_{n}\right) \in(\mathbb{R} / \mathbb{Z})^{n}$. Let $\mathbf{e}_{i} \in$ $H^{1}\left(T^{n} ; \mathbb{Z}\right)$ be the element represented by $d t_{i}$ in de Rham cohomology, where $t_{i}$ is the coordinate of the $i$-th factor of $\left(S^{1}\right)^{n}$. (Here we identify $S^{1}$ with $\mathbb{R} / \mathbb{Z}$.) The elements $\mathbf{e}_{i}, i=1, \ldots, n$ form a basis of $H^{1}\left(T^{n} ; \mathbb{Z}\right) \cong \mathbb{Z}^{n}$. Since the $T^{n}$ action on $L(\mathbf{u})$ is free and transitive, we may identify $H^{1}\left(T^{n} ; \mathbb{Z}\right)=$ $H^{1}(L(\mathbf{u}) ; \mathbb{Z})$. Hence we have a basis $\mathbf{e}_{i}, i=1, \ldots, n$ of $H^{1}(L(\mathbf{u}) ; \mathbb{Z})$.

Let $b \in H^{1}\left(L(\mathbf{u}) ; \Lambda_{0}\right)$. We can write $b=\sum_{i=1}^{n} x_{i} \mathbf{e}_{i}$. Hence we take $\left(x_{1}, \ldots, x_{n}\right)$ as a coordinate of $H^{1}\left(L(\mathbf{u}) ; \Lambda_{0}\right)$. We also put $y_{i}^{\mathbf{u}}=e^{x_{i}}$.

REMARK 5.1. The expression $e^{x_{i}}$ determines an element of $\Lambda_{0}$ in case $x_{i} \in \Lambda_{0}$ as follows. We write $x_{i}=x_{i, 0}+x_{i,+}$ where $x_{i, 0} \in \mathbb{C}$ and $x_{i,+} \in \Lambda_{+}$. Then we put

$$
y_{i}^{\mathbf{u}}=e^{x_{i}}=e^{x_{i, 0}} \sum_{k=0}^{\infty} x_{i,+}^{k} / k !
$$

Note $e^{x_{i, 0}} \in \mathbb{C}$ is defined as usual. The sum $\sum_{k=0}^{\infty} x_{i,+}^{k} / k$ ! converges in $\mathfrak{v}_{T^{-}}$ topology.

Now we consider a toric manifold $X$ with its moment polytope $P$. We consider affine functions $\ell_{j}(j=1, \ldots, m)$. We define $v_{j, i} \in \mathbb{Z}$ as in Properties 4.1 1). We define

$$
z_{j}=T^{\ell_{j}(\mathbf{u})}\left(y_{1}^{\mathbf{u}}\right)^{v_{j, 1}} \ldots\left(y_{n}^{\mathbf{u}}\right)^{v_{j, n}}
$$

TheOREM 5.2. $\quad 1) H^{1}\left(L(\mathbf{u}) ; \Lambda_{0}\right)$ is contained in $\widehat{\mathcal{M}}_{\text {weak }}\left(H\left(L ; \Lambda_{0}\right)\right.$; $\left.\Lambda_{0}\right)$.

2) Let $b=\sum x_{i} \mathbf{x}_{i} \in H^{1}\left(L(\mathbf{u}) ; \Lambda_{0}\right)$. Then we have

$$
\mathfrak{P O}(b)=z_{1}+\cdots+z_{m}+\sum_{k=1}^{N} T^{\rho_{k}} P_{k}\left(z_{1}, \ldots, z_{m}\right) .
$$


Here $N \in \mathbb{Z}_{\geq 0}$ or $N=\infty$. The numbers $\rho_{k}>0$ are positive and real. In case $N=\infty$, the sequence of numbers $\rho_{k}$ goes to $\infty$ as $k$ goes to $\infty$. $P_{k}\left(z_{1}, \ldots, z_{m}\right)$ are monomials of $z_{1}, \ldots, z_{m}$ of degree $\geq 1$ with $\Lambda_{0}$ coefficient. We remark that $z_{j}$ is defined from $y_{i}^{\mathbf{u}}=e^{x_{j}}$ by (19).

3) If $X$ is Fano then $P_{k}$ are all zero.

4) The monomials $P_{k}$ and the numbers $\rho_{k}$ are independent of $\mathbf{u}$ and depends only on $X$.

Item 1) is [FOOO3] Proposition 4.3 plus the last line of [FOOO3] section 4 .

Item 2) is [FOOO3] Theorem 4.6 in the form (slightly) improved in [FOOO4] Theorem 3.4. In [FOOO3, FOOO4] this formula is written using $y_{i}^{\mathbf{u}}$ in place of $z_{j}$. But it is easy to see that they are the same by the identification (19). We use the result of Cho-Oh $[\mathbf{C O}]$ to calculate the term $z_{1}+\cdots+z_{m}$ in the right hand side of $(20)$.

Item 3) is [FOOO3] Theorem 4.5.

Item 4) follows from [FOOO3] Lemma 11.7.

Sketch of the Proof. The linear terms $z_{j}$ in (20) come from the contribution (that is $\mathfrak{m}_{k, \beta_{j}}(b, \ldots, b)$ ) of $\mathcal{M}_{1}^{\text {main }}\left(\beta_{j}\right)$ to $\mathfrak{m}_{k}(b, \ldots, b)$, where $\beta_{j} \in H_{2}(X, L(\mathbf{u}) ; \mathbb{Z})$ is as in (14). Its coefficient 1 is the degree of the map

$$
\mathrm{ev}_{0}: \mathcal{M}_{1}^{\operatorname{main}}\left(\beta_{j}\right) \rightarrow L(\mathbf{u})
$$

which is calculated by $[\mathbf{C O}]$.

The term $T^{\rho_{k}} P_{k}$ is a contribution of $\mathcal{M}_{1}^{\text {main }}(\beta)$ for some $\beta$. We will assume $\beta \neq 0$ in the rest of the argument.

We can use a $T^{n}$ equivariant multisection to define virtual fundamental chain. To see this we first observe that the $T^{n}$ action on $\mathcal{M}_{1}^{\text {main }}(\beta)$ is free. This is because $T^{n}$ action on $L(\mathbf{u})$ is free and (21) is $T^{n}$ equivariant. Therefore to find a transversal multisection we can proceed as follows. We first take the quotient with respect to $T^{n}$ action, next find transversal multisection on the quotient space and then lift it.

Let $\mathfrak{s}$ be a $T^{n}$ equivariant multisection which is transversal to 0 . Then $T^{n}$ acts freely on its zero set $\mathcal{M}_{1}^{\text {main }}(\beta)^{\mathfrak{s}}$. Therefore the dimension of $\mathcal{M}_{1}^{\text {main }}(\beta)^{\mathfrak{s}}$ is not smaller than $n$ if it is nonempty. We can show

$$
\operatorname{dim} \mathcal{M}_{1}^{\operatorname{main}}(\beta)^{\mathfrak{s}}=n+\mu(\beta)-2
$$

where $\mu: H_{2}(X, L(\mathbf{u}) ; \mathbb{Z}) \rightarrow \mathbb{Z}$ is the Maslov index. It implies that $\mu(\beta) \geq 2$ if $\mathcal{M}_{1}^{\text {main }}(\beta)^{\mathfrak{s}} \neq \emptyset$.

This is the key point of the proof.

REMARK 5.3. In case $X$ is Fano, $\mu(\beta) \geq 2$ automatically holds if $\mathcal{M}_{1}^{\text {main }}(\beta) \neq \emptyset$. But in non-Fano case this holds only after taking $T^{n}$ equivariant perturbation. 
Moreover $T^{n}$ equivariance implies that $\mathrm{ev}_{0}: \mathcal{M}_{1}^{\operatorname{main}}(\beta) \rightarrow L(\mathbf{u})$ is a submersion if $\mathcal{M}_{1}^{\text {main }}(\beta) \neq \emptyset$. Therefore we may use this $T^{n}$ equivariant $\mathfrak{s}$ to define $\mathfrak{m}_{k, \beta}$. Namely we do not need to use a continuous family of multisections in this case.

Now if $\operatorname{deg} b=1$ then

$$
\operatorname{deg} \mathfrak{m}_{k, \beta}(b, \ldots, b)=2-\mu(\beta) \leq 0 .
$$

Namely $\mathfrak{m}_{k, \beta}(b, \ldots, b)$ is either 0 or is proportional to the unit. This proves item 1).

To study $\mathfrak{m}_{k, \beta}(b, \ldots, b)$ for $\beta \neq \beta_{j}$, we again use the classification of $J$ holomorphic disks in $[\mathbf{C O}]$ to find that if $\mathcal{M}_{1}^{\text {main }}(\beta)$ is nonempty the homology class $\beta$ is decomposed to a sum of $\beta_{j}$ 's $(j=1, \ldots, m)$ and sphere bubbles. Therefore

$$
\beta=\beta_{j_{1}}+\cdots+\beta_{j_{e}}+\alpha_{1}+\cdots+\alpha_{f}
$$

where $b_{j_{k}}$ is one of $b_{j}$ 's and $\alpha_{i} \in H_{2}(X ; \mathbb{Z})$ is represented by $J$-holomorphic sphere. We put

$$
c_{\beta}=\operatorname{deg}\left[\operatorname{ev}_{0}: \mathcal{M}_{1}^{\operatorname{main}}(\beta)^{\mathfrak{s}} \rightarrow L(\mathbf{u})\right] .
$$

Here the right hand side is the mapping degree of the map ev 0 . It is welldefined since in case $\mu(\beta)=2$ the boundary of $\mathcal{M}_{1}^{\text {main }}(\beta)^{\mathfrak{s}}$ is empty. (This is because $\mathcal{M}_{1}^{\operatorname{main}}\left(\beta^{\prime}\right)^{\mathfrak{s}}$ is empty if $\mu\left(\beta^{\prime}\right) \leq 0, \beta^{\prime} \neq 0$.)

Then we can show that

$$
\sum_{k=0}^{\infty} \mathfrak{m}_{k, \beta}(b, \ldots, b)=c_{\beta} T^{\sum_{i=1}^{f}\left(\alpha_{i} \cap \omega\right) / 2 \pi} z_{j_{1}} \ldots z_{j_{e}} .
$$

Item 2) follows from this formula.

Item 3) follows from the fact that in the Fano case, $\mathcal{M}_{1}^{\operatorname{main}}(\beta) \neq \emptyset$ and $\mu(\beta)=2$ imply $\beta=\beta_{j}$ for some $j$.

Item 4) follows from the fact that $c_{\beta}$ is independent of $\mathbf{u}$.

REMARK 5.4. In the general situation, the filtered $A_{\infty}$ structure associated to a Lagrangian submanifold is well-defined only up to isomorphism. In particular potential function $\mathfrak{P O}$ is well-defined only up to a coordinate change. (Namely it may depend on the choice of perturbation etc.) However in our toric case we can use a $T^{n}$ equivariant perturbation $\mathfrak{s}$ and then $\mathfrak{P O}$ is well-defined as a function on $H^{1}\left(L(\mathbf{u}) ; \Lambda_{0}\right)$ without ambiguity. This is a consequence of well-definedness of $c_{\beta}$ and is [FOOO3] Lemma 11.7.

We have the following useful criterion which reduces computation of Floer cohomology to the critical point theory of potential function.

TheOREM 5.5. Let $b=\sum x_{i} \mathbf{e}_{i} \in H^{1}\left(L(\mathbf{u}) ; \Lambda_{0}\right)$. Then the following three conditions are equivalent. 
1) For each of $i=1, \ldots, n$ we have:

$$
\left.\frac{\partial \mathfrak{P O}}{\partial x_{i}}\right|_{b}=0
$$

2)

$$
H F\left((L(\mathbf{u}), b),(L(\mathbf{u}), b) ; \Lambda_{0}\right) \cong H\left(T^{n} ; \Lambda_{0}\right)
$$

3)

$$
H F((L(\mathbf{u}), b),(L(\mathbf{u}), b) ; \Lambda) \neq 0 \text {. }
$$

Sketch of The PROOF. By definition

$$
\mathfrak{P O}(b) \mathbf{e}=\sum_{k=0}^{\infty} \mathfrak{m}_{k}(b, \ldots, b) .
$$

We differentiate (22) by $x_{i}$. Then using $\partial b / \partial x_{i}=\mathbf{e}_{i}$ we obtain:

$$
\left.\frac{\partial \mathfrak{P O}}{\partial x_{i}}\right|_{b} \mathbf{e}=\sum_{k_{1}=0}^{\infty} \sum_{k_{2}=0}^{\infty} \mathfrak{m}_{k_{1}+k_{2}+1}(\underbrace{b, \ldots, b}_{k_{1}}, \mathbf{e}_{i}, \underbrace{b, \ldots, b}_{k_{2}})=\mathfrak{m}_{1}^{b}\left(\mathbf{e}_{i}\right) .
$$

Here the second equality is the definition of $\mathfrak{m}_{1}^{b}$.

Now we assume item 2). Then we have $\mathfrak{m}_{1}^{b}\left(\mathbf{e}_{i}\right)=0$. Therefore (23) implies item 1).

We next assume item 1$)$. Then (23) implies $\mathfrak{m}_{1}^{b}\left(\mathbf{e}_{i}\right)=0$. We use it together with the fact that $\mathbf{e}_{i}$ generates $H\left(L(\mathbf{u}) ; \Lambda_{0}\right)$ by cup product, and $A_{\infty}$ formula to prove that $\mathfrak{m}_{1}^{b}=0$. (See $[\mathbf{F O O O 3}]$ proof of Lemma 13.1.) Item 2) follows.

The equivalence between item 2) and item 3) is proved in [FOOO3] Remark 13.9.

To apply Theorems 5.2 and 5.5 for the calculation of Floer cohomology of $T^{n}$, we need some algebraic discussion, which is in order.

Let $y_{1}, \ldots, y_{n}$ be $n$ formal variables. We consider the ring $\Lambda\left[y_{1}, \ldots, y_{n}\right.$, $\left.y_{1}^{-1}, \ldots, y_{n}^{-1}\right]$ of Laurent polynomials of $n$ variables with $\Lambda$ coefficient. We write it as $\Lambda\left[y, y^{-1}\right]$ for simplicity.

Let $\mathbf{u}=\left(u_{1}, \ldots, u_{n}\right) \in P$. We put

$$
y_{i}^{\mathbf{u}}=T^{-u_{i}} y_{i} \in \Lambda\left[y, y^{-1}\right] .
$$

By an easy computation we have

$$
T^{\ell_{j}(\mathbf{u})}\left(y_{1}^{\mathbf{u}}\right)^{v_{j, 1}} \ldots\left(y_{n}^{\mathbf{u}}\right)^{v_{j, n}}=T^{\ell_{j}\left(\mathbf{u}^{\prime}\right)}\left(y_{1}^{\mathbf{u}^{\prime}}\right)^{v_{j, 1}} \ldots\left(y_{n}^{\mathbf{u}^{\prime}}\right)^{v_{j, n}} .
$$

for $\mathbf{u}, \mathbf{u}^{\prime} \in P$. Therefore (19) defines an elements $z_{j} \in \Lambda\left[y, y^{-1}\right]$ in a way independent of $\mathbf{u} \in P$.

We next introduce a family of valuations $\mathfrak{v}_{T}^{\mathbf{u}}$ on $\Lambda\left[y, y^{-1}\right]$ parametrized by $\mathbf{u} \in P$. 
Let $F \in \Lambda\left[y, y^{-1}\right]$. Then for each $\mathbf{u} \in \operatorname{Int} P$ there exists $F_{i_{1} \ldots i_{n}}^{\mathbf{u}} \in \Lambda$ for $i_{1}, \ldots, i_{n} \in \mathbb{Z}^{n}$ such that

$$
F=\sum_{i_{1}, \ldots, i_{n} \in \mathbb{Z}^{n}} F_{i_{1} \ldots i_{n}}^{\mathbf{u}}\left(y_{1}^{\mathbf{u}}\right)^{i_{1}} \cdots\left(y_{n}^{\mathbf{u}}\right)^{i_{n}} .
$$

Here only finitely many of $F_{i_{1} \ldots i_{n}}^{\mathbf{u}}$ are nonzero. So the right hand side is actually a finite sum.

\section{DEFINITION 5.6.}

$$
\mathfrak{v}_{T}^{\mathbf{u}}(F)=\inf \left\{\mathfrak{v}_{T}\left(F_{i_{1} \ldots i_{n}}^{\mathbf{u}}\right) \mid F_{i_{1} \ldots i_{n}}^{\mathbf{u}} \neq 0\right\},
$$

if $F \neq 0$ and $\mathfrak{v}_{T}^{\mathbf{u}}(0)=+\infty$.

$\mathfrak{v}_{T}^{\mathbf{u}}$ defines a valuation on $\Lambda\left[y, y^{-1}\right]$.

We denote the completion of $\Lambda\left[y, y^{-1}\right]$ with respect to $\mathfrak{v}_{T}^{\mathbf{u}}$ by $\Lambda\left\langle\left\langle y, y^{-1}\right\rangle\right\rangle^{\mathbf{u}}$.

By definition we have

$$
\mathfrak{v}_{T}^{\mathbf{u}}\left(z_{j}\right)=\ell_{j}(\mathbf{u}) \geq 0
$$

for $\mathbf{u} \in P$. The following lemma is its immediate consequence.

LEMMA 5.7. The right hand side of (20) converges with respect to $\mathfrak{v}_{T}^{\mathbf{u}}$ for any $\mathbf{u} \in P$.

We remark that according to the general theory described in section 3 , the potential function $\mathfrak{P O}$ associated to a Lagrangian submanifold $L(\mathbf{u})$ is a $\Lambda_{+}$valued function on $\widehat{\mathcal{M}}_{\text {weak }}\left(L(\mathbf{u}) ; \Lambda_{0}\right)$. By Theorem $5.2(1)$, we have the inclusion $H^{1}\left(L(\mathbf{u}) ; \Lambda_{0}\right) \subset \widehat{\mathcal{M}}_{\text {weak }}\left(L(\mathbf{u}) ; \Lambda_{0}\right)$. Since $x_{1}, \ldots, x_{n} \in \Lambda_{0}$ forms a coordinate of $H^{1}\left(L(\mathbf{u}) ; \Lambda_{0}\right)$ with respect to the basis $\mathbf{e}_{i}$, we may regard $\mathfrak{P O}$ restricted to $H^{1}\left(L(\mathbf{u}) ; \Lambda_{0}\right)$ as a function on $\left(x_{1}, \ldots, x_{n}\right) \in \Lambda_{0}^{n} \cong H^{1}\left(L(\mathbf{u}) ; \Lambda_{0}\right)$.

Then by Theorem 5.22 ) we have

$$
\mathfrak{P O}\left(x_{1}, \ldots, x_{n}\right)=\mathfrak{P O}\left(x_{1}^{\prime}, \ldots, x_{n}^{\prime}\right)
$$

if $x_{i}-x_{i}^{\prime} \in 2 \pi \sqrt{-1} \mathbb{Z}$ for each $i$. In other words, we may regard $\mathfrak{P D}$ as a function of $y_{i}^{\mathbf{u}}=e^{x_{i}}$. Note $x_{i} \in \Lambda_{0}$ implies that $y_{i}^{\mathbf{u}}-1 \in \Lambda_{+}$. We next extend the domain of $\mathfrak{P O}$ by using Theorem 5.22 ).

We put $\lambda_{j}=\ell_{j}(\mathbf{0})$. Then it is easy to see from definition that

$$
z_{j}=T^{\lambda_{j}} y_{1}^{v_{j, 1}} \ldots y_{n}^{v_{j, n}} \text {. }
$$

LEMmA 5.8. Let $\left(\mathfrak{y}_{1}, \ldots, \mathfrak{y}_{n}\right) \in(\Lambda \backslash\{0\})^{n}$. We assume

$$
\left(\mathfrak{v}_{T}\left(\mathfrak{y}_{1}\right), \ldots, \mathfrak{v}_{T}\left(\mathfrak{y}_{n}\right)\right) \in P .
$$

We put $\mathfrak{z}_{j}=T^{\lambda_{j}} \mathfrak{y}_{1}^{v_{j, 1}} \ldots \mathfrak{y}_{n}^{v_{j, n}}$. Then

$$
\mathfrak{z}_{1}+\cdots+\mathfrak{z}_{m}+\sum_{k=1}^{N} T^{\rho_{k}} P_{k}\left(\mathfrak{z}_{1}, \ldots, \mathfrak{z}_{m}\right) \in \Lambda_{+}
$$

converges as $N \rightarrow \infty$ with respect to the valuation $\mathfrak{v}_{T}$. 
Proof. (27) implies $\mathfrak{v}_{T}\left(\mathfrak{z}_{j}\right)=\ell_{j}(\mathbf{u}) \geq 0$. The lemma then follows easily from $\lim _{k \rightarrow \infty} \rho_{k}=\infty$ in the statement of Theorem $5.2(20)$.

We define

$$
\mathfrak{A}(P)=\left\{\left(\mathfrak{y}_{1}, \ldots, \mathfrak{y}_{n}\right) \in(\Lambda \backslash\{0\})^{n} \mid\left(\mathfrak{v}_{T}\left(\mathfrak{y}_{1}\right), \ldots, \mathfrak{v}_{T}\left(\mathfrak{y}_{n}\right)\right) \in P\right\} .
$$

By Lemma 5.8 we may regard $\mathfrak{P O}$ as a function

$$
\mathfrak{P O}: \mathfrak{A}(P) \rightarrow \Lambda_{+} \cdot
$$

We remark that $\mathfrak{A}(P)$ is not a manifold. So we can not define differentiation of $\mathfrak{P O}$ in the sense of usual calculus. Instead we will define it as follows. We remark that $z_{j}$ and $P_{k}\left(z_{1}, \ldots, z_{m}\right)$ are Laurent monomials of $y_{1}, \ldots, y_{n}$ with $\Lambda_{0}$ coefficient. So we can differentiate it by $y_{i}$ in an obvious way. Moreover

$$
y_{i} \frac{\partial}{\partial y_{i}} P_{k}\left(z_{1}, \ldots, z_{m}\right)
$$

is again a monomial of $z_{1}, \ldots, z_{m}$ with $\Lambda_{0}$ coefficient. Therefore for $\mathfrak{y}=$ $\left(\mathfrak{y}_{1}, \ldots, \mathfrak{y}_{n}\right) \in \mathfrak{A}(P)$ the limit

$$
\lim _{N \rightarrow \infty}\left(\mathfrak{y}_{i} \frac{\partial z_{1}}{\partial y_{i}}(\mathfrak{y})+\cdots+\mathfrak{y}_{i} \frac{\partial z_{m}}{\partial y_{i}}(\mathfrak{y})+\sum_{k=1}^{N} T^{\rho_{k}} \mathfrak{y}_{i} \frac{\partial P_{k}}{\partial y_{i}}\left(\mathfrak{z}_{1}, \ldots, \mathfrak{z}_{m}\right)\right)
$$

converges. (Here we put $\mathfrak{z}_{j}=T^{\lambda_{j}} \mathfrak{y}_{1}^{v_{j, 1}} \ldots \mathfrak{y}_{n}^{v_{j, n}}$.) We write its limit as

$$
\mathfrak{y}_{i} \frac{\partial \mathfrak{P O}}{\partial y_{i}}(\mathfrak{y}) \text {. }
$$

Thus we have defined

$$
y_{i} \frac{\partial \mathfrak{P O}}{\partial y_{i}}: \mathfrak{A}(P) \rightarrow \Lambda_{+}
$$

We now have the following:

Theorem 5.9. For $\mathbf{u} \in \operatorname{Int} P$ the following two conditions are equivalent.

1) There exists $b \in H^{1}\left(L(\mathbf{u}) ; \Lambda_{0}\right)$ such that

$$
H F\left((L(\mathbf{u}), b),(L(\mathbf{u}), b) ; \Lambda_{0}\right) \cong H\left(T^{n} ; \Lambda_{0}\right) .
$$

2) There exists $\mathfrak{y}=\left(\mathfrak{y}_{1}, \ldots, \mathfrak{y}_{n}\right) \in \mathfrak{A}(P)$ such that

$$
\begin{gathered}
\qquad \begin{array}{c}
\mathfrak{y}_{i} \frac{\partial \mathfrak{P O}}{\partial y_{i}}(\mathfrak{y})=0 \\
\text { for } i=1, \ldots, n \text { and that } \\
\left(\mathfrak{v}_{T}\left(\mathfrak{y}_{1}\right), \ldots, \mathfrak{v}_{T}\left(\mathfrak{y}_{n}\right)\right)=\mathbf{u} .
\end{array}
\end{gathered}
$$

Definition 5.10. We say that $L(\mathbf{u})$ is a strongly balanced if the Condition 1) (=Condition 2)) in Theorem 5.9 is satisfied. 
Proof. 2) $\Longrightarrow 1$ ): Let $\mathfrak{y}$ be as in 2). We put $y_{i}^{\mathbf{u}}=T^{-u_{i}} \mathfrak{y}_{i}$. Then $\mathfrak{v}_{T}\left(y_{i}^{\mathbf{u}}\right)=$ 0 . Therefore there exist $y_{i, 0}^{\mathbf{u}} \in \mathbb{C}$ and $y_{i,+}^{\mathbf{u}} \in \Lambda_{+}$such that $y_{i}^{\mathbf{u}}=y_{i, 0}^{\mathbf{u}}+y_{i,+}^{\mathbf{u}}$. We put $x_{i, 0}=\log \left(y_{i, 0}^{\mathbf{u}}\right)$ and

$$
\left.x_{i,+}=\log \left(1+\left(y_{i, 0}^{\mathbf{u}}\right)^{-1} y_{i,+}^{\mathbf{u}}\right)\right) .
$$

Note $\left(y_{i, 0}^{\mathbf{u}}\right)^{-1} y_{i,+}^{\mathbf{u}} \in \Lambda_{+}$. Therefore we can define the right hand side by the Taylor expansion of $\log (1+z)$.

We put $x_{i}=x_{i, 0}+x_{i,+}$ and $b=\sum_{i=1}^{m} x_{i} \mathbf{e}_{1}$. Then using Theorem 5.5 it is easy to see that 1 ) is satisfied.

1) $\Longrightarrow 2$ ): Let $b=\sum x_{i} \mathbf{e}_{i}$ be as in 1$)$. We put $\mathfrak{y}_{i}=T^{u_{i}} e^{x_{i}}$. It is easy to see that $\mathfrak{y}=\left(\mathfrak{y}_{1}, \ldots, \mathfrak{y}_{n}\right)$ satisfies $\mathfrak{y}_{i} \frac{\partial \mathfrak{P O}}{\partial y_{i}}(\mathfrak{y})=0$.

REMARK 5.11. It is easy to see that $y_{i}^{\mathbf{0}}=y_{i}$, where $\mathbf{0} \in \mathbb{R}^{n}$ is the origin. Note that the moment polytope $P$ is well-defined only up to parallel translation. Namely we can replace it by $P+\mathbf{u}$ for any $\mathbf{u} \in \mathbb{R}^{n}$, then $P+\mathbf{u}$ corresponds to the same toric manifold as $P$.

Thus the choice $y_{i}^{\mathbf{0}}=y_{i}$ is quite ad-hoc, and we may take any $y_{i}^{\mathbf{u}}$ in place of $y_{i}$ in our story. In fact the ring $\Lambda\left[y, y^{-1}\right]$ can be canonically identified with the Laurent polynomial rings over $y_{i}^{\mathbf{u}}(i=1, \ldots, n)$ using $y_{i}^{\mathbf{u}} \in \Lambda\left[y, y^{-1}\right]$.

On the other hand, the valuation $\mathfrak{v}_{T}^{\mathbf{u}}$ and the completion $\Lambda\left\langle\left\langle y, y^{-1}\right\rangle\right\rangle^{\mathbf{u}}$ is canonically associated to the Lagrangian submanifold $L(\mathbf{u})$.

The variables $y_{i}^{\mathbf{u}}$ also is defined in a way independent of the choice of the origin of the affine space in which $P$ is embedded.

In some reference such as [Aur1, HV] 'renormalization' is discussed. It seems that this process depends on the choice of the origin in the affine space $\mathbb{R}^{n}$. Namely it is related to the homothetic transformation $y_{i} \mapsto C y_{i}$ where $C \rightarrow \infty$.

As we mentioned above the choice of $\mathbf{0}$ is not intrinsic. More canonical way seems to be as follows. We consider each of $\mathbf{u}_{0}$ such that $H F\left(\left(L\left(\mathbf{u}_{0}\right), b\right)\right.$, $\left.\left(L\left(\mathbf{u}_{0}\right), b\right) ; \Lambda\right) \neq 0$ for some $b$. We then replace $P$ by $P-\mathbf{u}_{0}$, so this orbit $L\left(\mathbf{u}_{0}\right)$ becomes $L(\mathbf{0})$. We now use $y_{i} \mapsto C y_{i}$ to 'renormalize'.

Thus there exists a 'renormalization' for each such $\mathbf{u}_{0}$. This process of 'renormalization' seems to be related to the study of leading term equation, which we discuss in section 9 .

\section{Examples 1}

EXAmple 6.1. We first consider the case of $\mathbb{C} P^{n}$. We use (16) and Theorem 5.22 ), 3) to obtain

$$
\mathfrak{P O}=z_{1}+\cdots+z_{n}+z_{0}=y_{1}+\cdots+y_{n}+T\left(y_{1} \ldots y_{n}\right)^{-1} \text {. }
$$

Therefore the equation (29) becomes

$$
0=y_{i} \frac{\partial \mathfrak{P O}}{\partial y_{i}}=y_{i}-T\left(y_{1} \ldots y_{n}\right)^{-1} \text {. }
$$


The solutions are

$$
y_{1}=\cdots=y_{n}=T^{1 /(n+1)} \exp (2 \pi \sqrt{-1} k /(n+1))
$$

where $k=0,1, \ldots, n$. The valuation of $y_{i}$ are $1 /(n+1)$. Thus $\mathbf{u}_{0}=(1 /(n+$ $1), \ldots, 1 /(n+1))$ is the unique strongly balanced fiber.

ExAmple 6.2. We next consider $X(\alpha)$, one point blow up of $\mathbb{C} P^{2}$ as in Example 4.3. Using the discussion in Example 4.3 and Theorem 5.2 2), 3) we obtain

$$
\mathfrak{P O}=y_{1}+y_{2}+T\left(y_{1} y_{2}\right)^{-1}+T^{1-\alpha} y_{2}^{-1} .
$$

The equation (29) becomes

$$
1-T y_{1}^{-2} y_{2}^{-1}=0, \quad 1-T y_{1}^{-1} y_{2}^{-2}-T^{1-\alpha} y_{2}^{-2}=0 .
$$

By eliminating $y_{2}=T y_{1}^{-2}$ we obtain

$$
y_{1}^{4}+T^{\alpha} y_{1}^{3}-T^{\alpha+1}=0 .
$$

We put $u_{1}=\mathfrak{v}_{T}\left(y_{1}\right)$.

(Case 1) $u_{1}<\alpha$.

We take $\mathfrak{v}_{T}$ of $(30)$ and obtain $4 u_{1}=\alpha+1$. Namely $u_{1}=(\alpha+1) / 4$. $u_{1}>\alpha$ then implies $\alpha>1 / 3$.

Conversely if $\alpha>1 / 3$ and $u_{1}=(\alpha+1) / 4$ we put $y_{1}=T^{u_{1}} y$ then (30) becomes

$$
y^{4}+T^{(3 \alpha-1) / 4} y^{3}-1=0 .
$$

Since $(3 \alpha-1) / 4>0$, this equation has 4 simple roots $y$ which are congruent to $\pm 1, \pm \sqrt{-1}$ modulo $\Lambda_{+}$, respectively.

(Case 2) $u_{1}>\alpha$.

We take $\mathfrak{v}_{T}$ of (30) and have $3 u_{1}+\alpha=\alpha+1$. Namely $u_{1}=1 / 3 . u_{1}>\alpha$ then implies $\alpha<1 / 3$.

Conversely if $\alpha<1 / 3$ and $u_{1}=1 / 3$ we put $y_{1}=T^{1 / 3} y$ then (30) becomes

$$
T^{1 / 3-\alpha} y^{4}+y^{3}-1=0 .
$$

This equation has 3 simple roots $y$ which are congruent to $1, e^{2 \pi \sqrt{-1} / 3}$, $e^{4 \pi \sqrt{-1} / 3}$ modulo $\Lambda_{+}$, respectively.

(Case 3) $u_{1}=\alpha$.

We put $y_{1}=T^{u_{1}} y$. Then $\mathfrak{v}_{T}(y)=0$ and we have

$$
y^{3}(1+y)-T^{1-3 \alpha}=0 .
$$

(Case 3-1) $\alpha=1 / 3$.

In this case there exist exactly 4 roots $y \in \mathbb{C}$ of $(31)$.

(Case $3-2) \alpha \neq 1 / 3$.

By $(31) \alpha<1 / 3$. Then $\mathfrak{v}_{T}(1+y)=1-3 \alpha$. We put $y=T^{1-3 \alpha} w-1$. Then $\mathfrak{v}_{T}(w)=0$. Then (31) becomes

$$
\left(1-T^{1-3 \alpha} w\right)^{3} w+1=0 .
$$


There is one root of this equation with $w \equiv-1$ modulo $\Lambda_{+}$. Three other roots do not satisfy $\mathfrak{v}_{T}(w)=0$. Thus there exists one solution in this case such that $u_{1}=\mathfrak{v}_{T}\left(y_{1}\right)=\alpha$.

In sum we have the following.

If $\alpha<1 / 3$ there exists one solution with $u_{1}=\mathfrak{v}_{T}\left(y_{1}\right)=\alpha$ and three solutions with $u_{1}=1 / 3$. Note $u_{2}=\mathfrak{v}_{T}\left(y_{2}\right)=1-2 u_{1}$. Therefore $L(\alpha, 1-2 \alpha)$ and $L(1 / 3,1 / 3)$ are the strongly balanced fibers.

If $\alpha \geq 1 / 3$ we have 4 solutions with $u_{1}=(\alpha+1) / 4, u_{2}=(1-\alpha) / 2$. Namely there is exactly one strong balanced fiber $L((\alpha+1) / 4,(1-\alpha) / 2)$.

In this section we discuss the Fano case only, where we can explicitly calculate $\mathfrak{P O}$. The non-Fano case will be discussed in section 10.

In the case of Example 6.1 and 6.2, McDuff [Mc] proved that all the $T^{n}$ orbits where Floer cohomology vanish for all choices of $b$, are displaceable by Hamiltonian diffeomorphism.

However there is an example of toric surface and its $T^{2}$ orbit, such that one can not displace it from itself by the method of $[\mathbf{M c}]$ but all the known versions of Floer cohomology over $\Lambda$ vanish for this $T^{2}$ orbit. (See [Mc] Lemma 4.4.) We do not know whether they are displaceable or not.

\section{Open-closed Gromov-Witten theory and operators $\mathfrak{q}$}

In this section, we discuss the operator $\mathfrak{q}$ introduced in [FOOO1] section 3.8. Let $(X, \omega)$ be a symplectic manifold and $L$ its Lagrangian submanifold as in section 3 . Let $h_{1}, \ldots, h_{k}$ be differential forms on $L$ and $g_{1}, \ldots, g_{\ell}$ differential forms on $X$. Let $\beta \in H_{2}(X, L ; \mathbb{Z})$. We define

$$
\begin{aligned}
& \mathfrak{q}_{\ell, k, \beta}\left(g_{1}, \ldots, g_{\ell} ; h_{1}, \ldots, h_{k}\right) \\
& \quad=\frac{1}{\ell !} \mathrm{ev}_{0 !}\left(\left(\mathrm{ev}_{1}^{+}, \ldots, \mathrm{ev}_{\ell}^{+}, \mathrm{ev}_{1}, \ldots, \mathrm{ev}_{k}\right)^{*}\left(g_{1} \times \cdots \times g_{\ell} \times h_{1} \times \cdots \times h_{k}\right) .\right.
\end{aligned}
$$

We also put

$$
\mathfrak{q}_{0 ; 1 ; 0}(h)=(-1)^{n} d h
$$

We remark that $g_{1} \times \cdots \times g_{\ell} \times h_{1} \times \cdots \times h_{k}$ is a differential form on $X^{\ell} \times L^{k}$ and its pull back is a differential form on $\mathcal{M}_{k+1 ; \ell}^{\text {main }}(\beta)$. The map ev 0 ! is integration along fiber by the map $\operatorname{ev}_{0}: \mathcal{M}_{k+1 ; \ell}^{\text {main }}(\beta) \rightarrow L$. More precisely we use a continuous family of perturbations in the same way as we defined $\mathfrak{m}_{k}$ in section 3 .

We then put

$$
\mathfrak{q}_{\ell, k}=\sum_{\beta \in H_{2}(X, L ; \mathbb{Z})} T^{(\beta \cap \omega) / 2 \pi} \mathfrak{q}_{\ell, k, \beta}
$$

It defines a map

$$
\mathfrak{q}_{\ell ; k}: E_{\ell}\left(\Omega(X)[2] \otimes \Lambda_{0}\right) \otimes B_{k}\left(\Omega(L)[1] \otimes \Lambda_{0}\right) \rightarrow \Omega(L)[1] \otimes \Lambda_{0} .
$$


This operator has the following properties. We omit the suffix $\ell, k$ in $\mathfrak{q}_{\ell ; k}$ and write $\mathfrak{q}$ in the formula below. We use the convention (6) introduced at the end of subsection 2.1.

Theorem 7.1. $\quad$ 1) Let $\mathbf{x} \in B_{k}\left(\Omega(L)[1] \otimes \Lambda_{0}\right), \mathbf{y} \in E_{\ell}\left(\Omega(X)[2] \otimes \Lambda_{0}\right)$. Suppose $\mathbf{y}$ is a linear combination of the elements of the form $y_{1} \otimes \cdots \otimes y_{\ell}$ where each of $y_{i}$ are closed forms. We then have the following:

$$
\begin{aligned}
0 & =\sum_{c_{1}, c_{2}}(-1)^{*} \mathfrak{q}\left(\mathbf{y}_{c_{1}}^{2 ; 1} ; \mathbf{x}_{c_{2}}^{3 ; 1} \otimes \mathfrak{q}\left(\mathbf{y}_{c_{1}}^{2 ; 2} ; \mathbf{x}_{c_{2}}^{3 ; 2}\right) \otimes \mathbf{x}_{c_{2}}^{3 ; 3}\right) \\
\text { where } * & =\operatorname{deg}^{\prime} \mathbf{x}_{c_{2}}^{3 ; 1}+\operatorname{deg}^{\prime} \mathbf{x}_{c_{2}}^{3 ; 1} \operatorname{deg} \mathbf{y}_{c_{1}}^{2 ; 2}+\operatorname{deg} \mathbf{y}_{c_{1}}^{2 ; 1} . \\
\text { 2) If } \mathbf{y}=1 & \in E_{0}\left(\Omega(X)[2] \otimes \Lambda_{0}\right)=\Lambda_{0} \text { then }
\end{aligned}
$$

3) Let $\mathbf{e}=\operatorname{PD}([L])$ be the Poincaré dual to the fundamental class of $L$. Let $\mathbf{x}_{i} \in B\left(\Omega(L)[1] \otimes \Lambda_{0}\right)$ and we put $\mathbf{x}=\mathbf{x}_{1} \otimes \mathbf{e} \otimes \mathbf{x}_{2} \in B(\Omega(L)[1] \otimes$ $\left.\Lambda_{0}\right)$. Then

$$
\mathfrak{q}(\mathbf{y} ; \mathbf{x})=0
$$

except the following case:

$$
\mathfrak{q}(1 ; \mathbf{e} \otimes x)=(-1)^{\operatorname{deg} x} \mathfrak{q}(1 ; x \otimes \mathbf{e})=x,
$$

where $x \in \Omega(L)[1] \otimes \Lambda_{0}=B_{1}\left(\Omega(L)[1] \otimes \Lambda_{0}\right)$.

4) There exists a discrete submonoid $G=\left\{\lambda_{i} \mid i=0,1,2, \ldots\right\}$ such that

$$
\mathfrak{q}_{\ell, k}=\sum_{i=1}^{\infty} T^{\lambda_{i}} \mathfrak{q}_{\ell, k, i}
$$

where $\mathfrak{q}_{\ell, k, i}: E_{\ell}(\Omega(X)[2]) \otimes B_{k}(\Omega(L)[1]) \rightarrow \Omega(L)[1]$.

5) Let $i: L \rightarrow X$ be the inclusion and $y \in \Omega(X) \otimes \Lambda_{0}$. Then

$$
\mathfrak{q}_{1,0}(y, 1) \equiv i^{*}(y) \quad \bmod \Omega(L) \otimes \Lambda_{+} .
$$

REMARK 7.2. Formula (33) above implies that the operator $\mathfrak{q}$ (after modifying the sign appropriately) define a homomorphism $E \mathcal{A}[2] \rightarrow H H^{*}(L ; \Lambda)$ to the Hochschild cohomology of de Rham cohomology ring of $L$. See [FOOO1] Section 7.4.

This is de Rham version of [FOOO1] Theorem 3.8.32. Namely item 1) is [FOOO1] (3.8.33), Item 2) is [FOOO1] Theorem 3.8.32 (3). Item 3) is [FOOO1] (3.8.34.2). Item 4) follows immediately from definition. Item 5) follows from [FOOO1] (3.8.34).

Let $\mathfrak{b} \in \Omega^{\text {even }}(X) \otimes \Lambda_{+}$and $b \in \Omega^{\text {odd }}(L) \otimes \Lambda_{+}$. Suppose $d \mathfrak{b}=0$. We put $\mathbf{b}=(\mathfrak{b}, b)$ and define

$$
\mathfrak{m}_{k}^{\mathbf{b}}: B_{k}\left(\Omega(L)[1] \otimes \Lambda_{0}\right) \rightarrow \Omega(L)[1] \otimes \Lambda_{0}
$$


by

$$
\begin{aligned}
\mathfrak{m}_{k}^{\mathbf{b}}\left(x_{1}, \ldots, x_{k}\right) \\
\quad=\sum_{\ell=0}^{\infty} \sum_{m_{0}=0}^{\infty} \cdots \sum_{m_{k}=0}^{\infty} \mathfrak{q}_{\ell, k}(\mathfrak{b}^{\ell} ; \underbrace{b, \ldots, b}_{m_{0}}, x_{1}, \underbrace{b, \ldots, b}_{m_{1}}, \ldots, x_{k}, \underbrace{b, \ldots, b}_{m_{k}}) .
\end{aligned}
$$

It is easy to see that $\left\{\mathfrak{m}_{k}^{\mathbf{b}} \mid k=0,1,2, \ldots\right\}$ defines a unital and gapped filtered $A_{\infty}$ structure.

We define $\widehat{\mathcal{M}}_{\text {def,weak }}(L)$ as the set of all $\mathbf{b}=(\mathfrak{b}, b)$ such that

$$
\mathfrak{m}_{0}^{\mathbf{b}}(1)=c \mathbf{e} .
$$

Here $\mathbf{e}=1 \in \Omega^{0}(L)$.

If $\mathbf{b} \in \widehat{\mathcal{M}}_{\text {def,weak }}(L)$ then we have

$$
\mathfrak{m}_{1}^{\mathbf{b}} \circ \mathfrak{m}_{1}^{\mathbf{b}}=0 .
$$

Definition 7.3. For $\mathbf{b} \in \widehat{\mathcal{M}}_{\text {def,weak }}(L)$, we define Floer cohomology with bulk deformation by

$$
H F\left((L, \mathbf{b}),(L, \mathbf{b}) ; \Lambda_{0}\right) \cong \frac{\operatorname{Ker} \mathfrak{m}_{1}^{\mathbf{b}}}{\operatorname{Im} \mathfrak{m}_{1}^{\mathbf{b}}} .
$$

$H F((L, \mathbf{b}),(L, \mathbf{b}) ; \Lambda)$ is defined by taking $\otimes_{\Lambda_{0}} \Lambda$.

We define the potential function $\mathfrak{P O}: \widehat{\mathcal{M}}_{\text {def,weak }}(L) \rightarrow \Lambda_{+}$by the equation

$$
\mathfrak{P O e}=\mathfrak{m}_{0}^{\mathbf{b}}(1) .
$$

We also put $\mathfrak{P O}^{\mathfrak{b}}(b)=\mathfrak{P O}(\mathfrak{b}, b)$.

If $H F((L, \mathbf{b}),(L, \mathbf{b}) ; \Lambda) \neq 0$ then $L$ is non-displaceable. This is [FOOO4] Proposition 3.15 which is proved in [FOOO4] section 8.

\section{Floer cohomology with bulk deformation in the toric case}

Now we apply the construction explained in the last section to the case of toric manifolds. In this section we use cycles (submanifolds) rather than differential forms to represent the (co)homology classes of ambient manifold $X$, by a reason we will mention in Remark 8.3.

Let $D_{1}, \ldots, D_{m}$ be the irreducible components of toric divisors. Let $J=$ $\left\{j_{1}, \ldots, j_{k}\right\} \subseteq\{1, \ldots, m\}$. If $D_{J}=D_{j_{1}} \cap \cdots \cap D_{j_{k}}$ is non-empty, it is a (real) codimension $2 k$ submanifold of $X$. We include the case $J=\emptyset$. In that case $D_{J}=X$. We denote by $\mathcal{A}$ the free abelian group generated by $D_{J}$. We put cohomology degree on it. Namley $\operatorname{deg} D_{J}=2 k$ if codimension of $D_{J}$ is $2 k$. We define $\mathcal{A}\left(\Lambda_{0}\right)=\mathcal{A} \otimes \Lambda_{0}$.

There is an obvious homomorphism

$$
\mathcal{A} \rightarrow H^{*}(X ; \mathbb{Z})
$$


which is surjective but not injective. We write the generator of $\mathcal{A}$ as $\mathbf{p}_{i}$, $(i=0, \ldots, B)$, where $\mathbf{p}_{0}=X$ and $\mathbf{p}_{i}=D_{i}$ for $i=1, \ldots, m$ are degree 2 classes. For $I=\left(i_{1}, \ldots, i_{\ell}\right) \in\{1, \ldots, B\}^{\ell}$ we put

$$
\mathbf{p}_{I}=\mathbf{p}_{i_{1}} \otimes \cdots \otimes \mathbf{p}_{i_{\ell}}, \quad\left[\mathbf{p}_{I}\right]=\frac{1}{\ell !} \sum_{\sigma \in \mathfrak{S}_{\ell}} \mathbf{p}_{i_{\sigma(1)}} \otimes \cdots \otimes \mathbf{p}_{i_{\sigma(\ell)}} \in E_{\ell} \mathcal{A}[2] .
$$

Here $\mathfrak{S}_{\ell}$ is the symmetric group of order $\ell$ !.

Let $\mathbf{u} \in \operatorname{Int} P, \beta \in H_{2}(X, L(\mathbf{u}) ; \mathbb{Z})$ and $I \in\{1, \ldots, B\}^{\ell}$. We define:

$$
\mathcal{M}_{k+1, \ell}^{\text {main }}\left(\beta, \mathbf{p}_{I}\right)=\mathcal{M}_{k+1, \ell}^{\operatorname{main}}(\beta)_{\left(\mathrm{ev}_{1}^{+}, \ldots, \mathrm{ev}_{\ell}^{+}\right)} \times_{X^{\ell}} \mathbf{p}_{I} .
$$

Note $\mathrm{ev}_{i}^{+}$are evaluation maps at interior marked points. We then still have evaluation maps at boundary marked points:

$$
\mathrm{ev}=\left(\mathrm{ev}_{0}, \ldots, \mathrm{ev}_{k}\right): \mathcal{M}_{k+1, \ell}^{\text {main }}\left(\beta, \mathbf{p}_{I}\right) \rightarrow L^{k+1} .
$$

We use it to define an operator

$$
\mathfrak{q}_{\ell, k ; \beta}: E_{\ell} \mathcal{A}[2] \otimes B_{k} H(L(\mathbf{u}) ; \mathbb{C})[1] \rightarrow H(L(\mathbf{u}) ; \mathbb{C})[1]
$$

as follows. We remark that there is a transitive and free action of $T^{n}$ on $L(\mathbf{u})$. We put a $T^{n}$ invariant metric on $L(\mathbf{u})$. Harmonic forms with respect to this metric are nothing but the $T^{n}$ invariant differential forms. We identify the cohomology group $H(L(\mathbf{u}) ; \mathbb{C})$ with the set of the $T^{n}$ invariant forms on $L(\mathbf{u})$ from now on.

Let $h_{1}, \ldots, h_{k} \in H(L(\mathbf{u}) ; \mathbb{C})$. The pull-back

$$
\left(\mathrm{ev}_{1}, \ldots, \mathrm{ev}_{k}\right)^{*}\left(h_{1} \times \cdots \times h_{k}\right)
$$

is a differential form on $\mathcal{M}_{k+1, \ell}^{\text {main }}\left(\beta, \mathbf{p}_{I}\right)$. We use integration along fiber of the evaluation map $\mathrm{ev}_{0}: \mathcal{M}_{k+1, \ell}^{\operatorname{main}}\left(\beta, \mathbf{p}_{I}\right) \rightarrow L$ and define:

$$
\mathfrak{q}_{\ell, k ; \beta}\left(\left[\mathbf{p}_{I}\right] ; h_{1} \times \cdots \times h_{k}\right)=\mathrm{ev}_{0 !}\left(\mathrm{ev}_{1}, \ldots, \mathrm{ev}_{k}\right)^{*}\left(h_{1} \times \cdots \times h_{k}\right) .
$$

We can perform all the constructions in a $T^{n}$ equivariant way. So the right hand side is a $T^{n}$ invariant differential form, which we identify with an element of cohomology group.

REMARK 8.1. To define integration along the fiber, we need the map $\mathrm{ev}_{0}: \mathcal{M}_{k+1, \ell}^{\text {main }}\left(\beta, \mathbf{p}_{I}\right) \rightarrow L$ to be a submersion. We also need the moduli space to be transversal after taking an appropriate perturbation.

We can do so by using multisection in the same way as section 5 as follows. We remark that the fiber product moduli space $\mathcal{M}_{k+1, \ell}^{\text {main }}\left(\beta, \mathbf{p}_{I}\right)$ has a Kuranishi structure. The group $T^{n}$ acts on it. Moreover the $T^{n}$ action is free. (This is because $\mathrm{ev}_{0}$ is $T^{n}$ equivariant and the $T^{n}$ action on $L(\mathbf{u})$ is free.) Thus by the same argument as we explained during the proof of Theorem 5.2 , we can take multisection $\mathfrak{s}$ which is $T^{n}$ equivariant and transversal to 0 . Then $\mathrm{ev}_{0}: \mathcal{M}_{k+1, \ell}^{\text {main }}\left(\beta, \mathbf{p}_{I}\right)^{\mathfrak{s}} \rightarrow L(\mathbf{u})$ automatically becomes a submersion if $\mathcal{M}_{k+1, \ell}^{\text {main }}\left(\beta, \mathbf{p}_{I}\right)^{\mathfrak{s}}$ is nonempty. 
We can also choose our perturbation so that it is invariant under the permutation of the interior marked points so descents to $E_{\ell} \mathcal{A}[2]$. Therefore the right hand side of (43) depends only on $\left[\mathbf{p}_{I}\right]$ rather than on $\mathbf{p}_{I}$.

We now define

$$
\mathfrak{q}_{\ell, k}: E_{\ell} \mathcal{A}\left(\Lambda_{0}\right)[2] \otimes B_{k} H\left(L(\mathbf{u}) ; \Lambda_{0}\right)[1] \rightarrow H\left(L(\mathbf{u}) ; \Lambda_{0}\right)[1]
$$

by

$$
\mathfrak{q}_{\ell, k}=\sum_{\beta \in H_{2}(X ; L(\mathbf{u}) ; \mathbb{Z})} T^{(\omega \cap \beta) / 2 \pi} \mathfrak{q}_{\ell, k ; \beta}
$$

In case we consider elements of $E_{\ell} \mathcal{A}\left(\Lambda_{0}\right)[2]$ which contain $\mathbf{p}_{0}$, the Poincaré dual to $[X]$, we define $\mathfrak{q}_{\ell, k}$ as follows:

$$
\mathfrak{q}_{1,0}\left(\mathbf{p}_{0} ; 1\right)=\mathbf{e}
$$

In all the other cases, $\mathfrak{q}_{\ell, k}$ is zero if the first factor $E_{\ell} \mathcal{A}\left(\Lambda_{0}\right)[2]$ contains $\mathbf{p}_{0}$.

Then our $\mathfrak{q}_{\ell, k}$ satisfies the conclusion of Theorem 7.1.

For $\mathbf{b}=(\mathfrak{b}, b) \in \mathcal{A}\left(\Lambda_{+}\right) \times H^{\text {odd }}\left(L(\mathbf{u}) ; \Lambda_{+}\right)$, we define $\mathfrak{m}_{k}^{\mathbf{b}}$ by $(36)$. It defines a unital gapped filtered $A_{\infty}$ structure on $H\left(L(\mathbf{u}) ; \Lambda_{0}\right)$.

Now we define

$$
\widehat{\mathcal{M}}_{\text {def,weak }}\left(L(\mathbf{u}) ; \Lambda_{+}\right) \subset \mathcal{A}\left(\Lambda_{+}\right) \times H^{\text {odd }}\left(L(\mathbf{u}) ; \Lambda_{+}\right)
$$

as the set of all $\mathbf{b}=(\mathfrak{b}, b) \in \mathcal{A}\left(\Lambda_{+}\right) \times H^{o d d}\left(L(\mathbf{u}) ; \Lambda_{+}\right)$such that $\mathfrak{m}_{0}^{\mathbf{b}}(1) \equiv 0$ $\bmod \Lambda_{+} \mathbf{e}$. In other words it is the set of $(\mathfrak{b}, b)$ such that

$$
\sum_{\ell=0}^{\infty} \sum_{k=0}^{\infty} \mathfrak{q}_{\ell ; k}\left(\mathfrak{b}^{\ell} ; b^{k}\right) \equiv 0 \quad \bmod \Lambda_{+} \mathbf{e} .
$$

We define the potential function $\mathfrak{P O}: \widehat{\mathcal{M}}_{\text {def,weak }}\left(L(\mathbf{u}) ; \Lambda_{+}\right) \rightarrow \Lambda_{+}$by

$$
\sum_{\ell=0}^{\infty} \sum_{k=0}^{\infty} \mathfrak{q}_{\ell ; k}\left(\mathfrak{b}^{\ell} ; b^{k}\right)=\mathfrak{P O}(\mathfrak{b} ; b) \mathbf{e} .
$$

Using a similar trick as the one used in section 5 we can extend the story to the cohomology groups with $\Lambda_{0}$ coefficient. Namely we obtain a MaurerCartan scheme

$$
\widehat{\mathcal{M}}_{\text {def,weak }}\left(L(\mathbf{u}) ; \Lambda_{0}\right) \subset \mathcal{A}\left(\Lambda_{0}\right) \times H^{\text {odd }}\left(L(\mathbf{u}) ; \Lambda_{0}\right)
$$

and Floer cohomology parametrized thereover. We also have a potential function

$$
\mathfrak{P O}: \widehat{\mathcal{M}}_{\text {def,weak }}\left(L(\mathbf{u}) ; \Lambda_{0}\right) \rightarrow \Lambda_{+} .
$$

Most of the stories in section 5 can be generalized to the current situation.

Theorem 8.2. $\quad$ 1) $\mathcal{A}\left(\Lambda_{0}\right) \times H^{1}\left(L(\mathbf{u}) ; \Lambda_{0}\right)$ is contained in $\widehat{\mathcal{M}}_{\text {def,weak }}\left(H\left(L ; \Lambda_{0}\right) ; \Lambda_{0}\right)$. 
2) Let $b=\sum x_{i} \mathbf{x}_{i} \in H^{1}\left(L(\mathbf{u}) ; \Lambda_{0}\right)$ and $\mathfrak{b} \in \mathcal{A}\left(\Lambda_{+}\right)$. Then we have

$$
\mathfrak{P O}(\mathfrak{b}, b)=z_{1}+\cdots+z_{m}+\sum_{k=1}^{N} T^{\rho_{k}} P_{k}\left(\mathfrak{b} ; z_{1}, \ldots, z_{m}\right) .
$$

Here $N \in \mathbb{Z}_{\geq 0}$ or $N=\infty$. The numbers $\rho_{k}$ are all positive and real. In case $N=\infty$, the sequence of numbers $\rho_{k}$ goes to $\infty$ as $k$ goes to $\infty . P_{k}\left(\mathfrak{b} ; z_{1}, \ldots, z_{m}\right)$ are monomials of $z_{1}, \ldots, z_{m}$ of degree $\geq 1$ with $\Lambda_{0}$ coefficient. (Here degree means that of monomials of $z_{i}$.) We remark that $z_{j}$ is defined from $y_{i}^{\mathbf{u}}=e^{x_{j}}$ by (19).

3) Let $b=\sum x_{i} \mathbf{x}_{i} \in H^{1}\left(L(\mathbf{u}) ; \Lambda_{0}\right)$ and $\mathfrak{b} \in \mathcal{A}\left(\Lambda_{0}\right)$.

$$
\begin{aligned}
\mathfrak{P O}(\mathfrak{b}, b)= & \mathfrak{c}_{1} z_{1}+\cdots+\mathfrak{c}_{m} z_{m}+P_{0}\left(\mathfrak{b} ; z_{1}, \ldots, z_{m}\right) \\
& +\sum_{k=1}^{N} T^{\rho_{k}} P_{k}\left(\mathfrak{b} ; z_{1}, \ldots, z_{m}\right) .
\end{aligned}
$$

$P_{0}\left(\mathfrak{b} ; z_{1}, \ldots, z_{m}\right)$ is a formal power series of $z_{1}, z_{2}, \ldots, z_{m}$ with $\Lambda_{0}$ coefficient such that each term has degree $\geq 2$. The numbers $\mathfrak{c}_{j}$ are defined as follows. Let $\mathfrak{b}=\sum w_{j} \mathbf{p}_{j}$. We put $w_{j} \equiv \bar{w}_{j} \bmod \Lambda_{+}$and $\bar{w}_{j} \in \mathbb{C}$. Then $\mathfrak{c}_{j}=e^{\bar{w}_{j}} \in \mathbb{C} \backslash\{0\}$. Other notations are the same as in (47).

4) The monomials $P_{k}$ and the numbers $\rho_{k}$ are independent of $\mathbf{u}$ and depends only on $X$ and $\mathfrak{b}$.

Item 1) is [FOOO4] Proposition 3.1. (In [FOOO4] Proposition 3.1 it is assumed that $\mathfrak{b} \in \mathcal{A}\left(\Lambda_{+}\right)$. It holds also for $\mathfrak{b} \in \mathcal{A}\left(\Lambda_{0}\right)$. See [FOOO4] section 11.)

Item 2) is [FOOO4] Theorem 3.4.

Item 3) follows from [FOOO4] sections 8 and 11. (Formulas (9.3), (11.1) etc.)

Item 4) follows from [FOOO4] Lemma 6.8.

The proof of Theorem 8.2 is similar to that of Theorem 5.2. We here mention only a few points. Let $I=\left(i_{1}, \ldots, i_{\ell}\right) \in\{1, \ldots, B\}^{\ell}$. We put $\mathbf{p}_{I}=$ $\mathbf{p}_{i_{1}} \otimes \cdots \otimes \mathbf{p}_{i_{\ell}}$. We have

$$
\operatorname{dim} \mathcal{M}_{1, \ell}^{\operatorname{main}}\left(\beta ; \mathbf{p}_{I}\right)=n-2+\mu(\beta)-\sum_{i=1}^{\ell}\left(\operatorname{deg} \mathbf{p}_{i}-2\right) .
$$

Here dim is the virtual dimension that is the dimension in the sense of Kuranishi structure. As we explained in Remark 8.1 the perturbed moduli space $\mathcal{M}_{1, \ell}^{\text {main }}\left(\beta ; \mathbf{p}_{I}\right)^{\mathfrak{s}}$ is empty if $(49)<n$.

REMARK 8.3. This is the reason why we use cycles $\mathbf{p}_{i}$ rather than differential forms on $X$ to represent cohomology classes of $X$. This point is crucial to prove item 1) in Theorem 8.2. 
In the case $(49)=n$ we define

$$
c(\beta ; I)=\operatorname{deg}\left[\operatorname{ev}_{0}: \mathcal{M}_{1, \ell}^{\operatorname{main}}\left(\beta ; \mathbf{p}_{I}\right)^{\mathfrak{s}} \rightarrow L(\mathbf{u})\right] \in \mathbb{Q} .
$$

Here and hereafter $\mathcal{M}_{1, \ell}^{\text {main }}\left(\beta ; \mathbf{p}_{I}\right)^{\mathfrak{s}}$ denotes the perturbation of the moduli space $\mathcal{M}_{1, \ell}^{\text {main }}\left(\beta ; \mathbf{p}_{I}\right)$. Namely it is the zero set of the multisection $\mathfrak{s}$. This zero set has a triangulation and each simplex of maximal degree comes with a weight $\in \mathbb{Q}$. Thus it has a virtual fundamental cycle. See [FOOO1] Section A1.

The number (50) is well-defined. Namely it is independent of the perturbation $\mathfrak{s}$ as far as it is $T^{n}$ equivariant. It is also independent of $\mathbf{u}$. ([FOOO4] Lemma 6.8.) The potential function is calculated by using $c(\beta ; I)$ as follows. Let $\vec{\ell}=\left(\ell_{1}, \ldots, \ell_{B}\right) \in \mathbb{Z}_{\geq 0}^{B}$. We put

$$
I(\vec{\ell})=(\underbrace{1, \ldots, 1}_{\ell_{1}}, \ldots, \underbrace{B, \ldots, B}_{\ell_{B}}) \in\{1, \ldots, B\}^{\sum_{i=1}^{B} \ell_{i}},
$$

and

$$
c(\beta ; \vec{\ell})=c(\beta ; I(\vec{\ell}))
$$

Let $\mathfrak{b}=\sum_{i=0}^{B} w_{i} \mathbf{p}_{i}$.

We define $\partial_{i}(\beta) \in \mathbb{Z}$ by

$$
\partial_{i}(\beta)=\left\langle\partial \beta, \mathbf{e}_{i}^{*}\right\rangle
$$

and put

$$
\left(y^{\mathbf{u}}\right)^{\partial \beta}=\left(y_{1}^{\mathbf{u}}\right)^{\partial_{1} \beta} \cdots\left(y_{n}^{\mathbf{u}}\right)^{\partial_{n} \beta}=T^{-\langle\partial \beta, \mathbf{u}\rangle} y_{1}^{\partial_{1} \beta} \cdots y_{n}^{\partial_{n} \beta} .
$$

Now we have

$$
\begin{aligned}
\mathfrak{P O}(\mathfrak{b}, b)= & w_{0}+\sum_{\beta \in H_{2}(X, L(\mathbf{u}) ; \mathbb{Z})} \sum_{\ell_{1}=0}^{\infty} \cdots \sum_{\ell_{B}=0}^{\infty} \\
& \times \frac{T^{(\beta \cap \omega) / 2 \pi}}{\ell_{1} ! \cdots \ell_{B} !} c(\beta ; \vec{\ell}) w_{1}^{\ell_{1}} \cdots w_{B}^{\ell_{B}}\left(y^{\mathbf{u}}\right)^{\partial \beta} .
\end{aligned}
$$

For the proof of (51) see [FOOO4] section 9.

(47) follows from $(51)$ and $c\left(\beta_{j} ;(0, \ldots, 0)\right)=1$. This follows from $[\mathbf{C O}]$. (See $[\mathbf{F O O O 4}]$ section 7.)

Theorem 5.5 is generalized to our situation without change. Namely we have the following theorem. Hereafter we put $\mathfrak{P O}^{\mathfrak{b}}(b)=\mathfrak{P O}(\mathfrak{b}, b)$.

TheOREM 8.4. Let $b=\sum x_{i} \mathbf{e}_{i} \in H^{1}\left(L(\mathbf{u}) ; \Lambda_{0}\right)$ and $\mathfrak{b} \in \mathcal{A}\left(\Lambda_{0}\right)$. Then the following three conditions are equivalent.

1) For each of $i=1, \ldots, n$ we have:

$$
\left.\frac{\partial \mathfrak{P O ^ { \mathfrak { b } }}}{\partial x_{i}}\right|_{b}=0
$$


2)

$$
H F\left((L(\mathbf{u}),(\mathfrak{b}, b)),(L(\mathbf{u}),(\mathfrak{b}, b)) ; \Lambda_{0}\right) \cong H\left(T^{n} ; \Lambda_{0}\right)
$$

3)

$$
H F((L(\mathbf{u}),(\mathfrak{b}, b)),(L(\mathbf{u}),(\mathfrak{b}, b)) ; \Lambda) \neq 0 .
$$

The proof is the same as the proof of Theorem 5.5 except some technical points, which we omit and refer [FOOO4].

The discussion in section 5 on the domain of the function $\mathfrak{P O}$ as a function of $y_{i}$ is also generalized.

We put:

$$
\mathfrak{A}(\stackrel{\circ}{P})=\left\{\left(\mathfrak{y}_{1}, \ldots, \mathfrak{y}_{n}\right) \in \Lambda^{n} \mid\left(\mathfrak{v}_{T}\left(\mathfrak{y}_{1}\right), \ldots, \mathfrak{v}_{T}\left(\mathfrak{y}_{n}\right)\right) \in \operatorname{Int} P\right\} .
$$

We remark that by Theorem $8.2 \mathfrak{P D}^{\mathfrak{b}}$ may be regarded as a function of $y_{1}, \ldots, y_{n}$.

Lemma 8.5. Let $\left(\mathfrak{y}_{1}, \ldots, \mathfrak{y}_{n}\right) \in \mathfrak{A}(\stackrel{\circ}{P})$. We put $\mathfrak{z}_{j}=T^{\lambda_{j}} \mathfrak{y}_{1}^{v_{j, 1}} \ldots \mathfrak{y}_{n}^{v_{j, n}}$. Then

$$
\mathfrak{z}_{1}+\cdots+\mathfrak{z}_{m}+P_{0}\left(\mathfrak{b} ; \mathfrak{z}_{1}, \ldots, \mathfrak{z}_{m}\right)+\sum_{k=1}^{N} T^{\rho_{k}} P_{k}\left(\mathfrak{b} ; \mathfrak{z}_{1}, \ldots, \mathfrak{z}_{m}\right) \in \Lambda_{+}
$$

converges as $N \rightarrow \infty$ with respect to the valutaion $\mathfrak{v}_{T}$.

In case $\mathfrak{b} \in \mathcal{A}\left(\Lambda_{+}\right)$where the term $P_{0}\left(\mathfrak{b} ; \mathfrak{z}_{1}, \ldots, \mathfrak{z}_{m}\right)$ is absent, we may relax the assumption to $\left(\mathfrak{y}_{1}, \ldots, \mathfrak{y}_{n}\right) \in \mathfrak{A}(P)$.

Thus we may regard $\mathfrak{P O}^{\mathfrak{b}}$ as a function either $: \mathfrak{A}(\stackrel{\circ}{P}) \rightarrow \Lambda_{+}$or : $\mathfrak{A}(P) \rightarrow \Lambda_{0}$.

We can define

$$
\mathfrak{y}_{i} \frac{\partial \mathfrak{P \mathfrak { D } ^ { \mathfrak { b } }}}{\partial y_{i}}
$$

in the same way as section 5 . It defines either a function $: \mathfrak{A}(\stackrel{\circ}{P}) \rightarrow \Lambda_{+}$or $: \mathfrak{A}(P) \rightarrow \Lambda_{0}$. Theorem 5.9 can be generalized as follows:

Theorem 8.6. For $\mathbf{u} \in \operatorname{Int} P, \mathfrak{b} \in \mathcal{A}\left(\Lambda_{0}\right)$, the following two conditions are equivalent.

1) There exists $b \in H^{1}\left(L(\mathbf{u}) ; \Lambda_{0}\right)$ such that

$$
H F\left((L(\mathbf{u}),(\mathfrak{b}, b)),(L(\mathbf{u}),(\mathfrak{b}, b)) ; \Lambda_{0}\right) \cong H\left(T^{n} ; \Lambda_{0}\right) .
$$

2) There exists $\mathfrak{y}=\left(\mathfrak{y}_{1}, \ldots, \mathfrak{y}_{n}\right) \in \mathfrak{A}(P)$ such that

$$
\mathfrak{y}_{i} \frac{\partial \mathfrak{P \mathfrak { D } ^ { \mathfrak { b } }}}{\partial y_{i}}(\mathfrak{y})=0
$$




$$
\begin{aligned}
& \text { for } i=1, \ldots, n \text { and that } \\
& \qquad\left(\mathfrak{v}_{T}\left(\mathfrak{y}_{1}\right), \ldots, \mathfrak{v}_{T}\left(\mathfrak{y}_{n}\right)\right)=\mathbf{u} .
\end{aligned}
$$

This is [FOOO4] Theorem 3.12.

\section{Leading term equation}

Theorem 8.6 provides a means of determining the Floer cohomology in terms of the potential function. The main obstacle to directly apply the theorem in practice is that we do not know how to calculate the extra terms $P_{k}\left(\mathfrak{b} ; z_{1}, \cdots, z_{n}\right)$ unless $X$ is Fano and $\mathfrak{b}$ has degree 2. (There has been some computation carried out in this direction for the nef case. See e.g. $[\mathbf{C L a}$.)

Fortunately to determine all the $T^{n}$ orbits $L(\mathbf{u})$ for which some Floer cohomology with bulk does not vanish, we do not need to calculate those terms. We will explain it in this section.

In this and the next sections we fix $\mathfrak{b}$ and $\mathbf{u}$ and consider $\mathfrak{P O}^{\mathfrak{b}}$ as a function of variables $y_{i}^{\mathbf{u}}$. In this section we write $\bar{y}_{i}$ instead of $y_{i}^{\mathbf{u}}$. We remark that $\mathfrak{v}_{T}^{\mathbf{u}}\left(\bar{y}_{i}\right)=0$ and

$$
z_{j}=T^{\ell_{j}(\mathbf{u})} \bar{y}_{1}^{v_{j, 1}} \cdots \bar{y}_{1}^{v_{j, n}}
$$

Definition 9.1. We denote the sum of linear terms $z_{j}$ 's in $\mathfrak{P D}^{\mathfrak{b}}$ by

$$
\mathfrak{P} \mathfrak{O}_{0}^{\mathfrak{b}}=\mathfrak{c}_{1} z_{1}+\cdots+\mathfrak{c}_{m} z_{m}=\sum_{j=1}^{m} T^{\ell_{j}(\mathbf{u})} \mathfrak{c}_{j} \bar{y}_{1}^{v_{j, 1}} \cdots \bar{y}_{n}^{v_{j, n}}
$$

and call it the leading order potential function. Here $\mathfrak{c}_{j}$ is defined as in Theorem 8.23$)$.

Note this function appears frequently in the literature (see [Gi1, HV, Iri1]), is denoted as $W$, and is called the (Landau-Ginzburg) superpotential.

REMARK 9.2. Note in our situation of toric manifold, superpotential in physics literature is basically the same as our potential function. However in other situation they may be different. For example in the case of Calabi-Yau 3 fold $X$ and its special Lagrangian submanifold $L$, our potential function is identically 0 . (In other words, if $b$ is a weak bounding chain then it is a bounding chain automatically.) On the other hand, the physisists' superpotential coincides with the invariant introduced in $[\mathbf{F u 4}]$.

We remark that the leading order potential function $\mathfrak{P O}_{0}^{\mathfrak{b}}$ is explicitly read off from the moment polytope $P$ and $\mathbf{u}$. The leading term equation we will define below depends only on leading order potential function and so is also explicitly calculable.

We renumber the values $\ell_{i}(\mathbf{u})$ according to its order. Namely we take $j(l, r) \in\{1, \ldots, m\}$ for $l=1, \ldots, K_{0}, r=1, \ldots, a\left(l^{\prime}\right)$ with the following conditions. 
Conditions 9.3. $\{1, \ldots, m\}$.

1) $\left\{j(l, r) \mid l=1, \ldots, K_{0}, r=1, \ldots, a(l)\right\}=$

2) $a(1)+\cdots+a\left(K_{0}\right)=m$.

3) $\ell_{j(l, r)}(\mathbf{u})=\ell_{j\left(l, r^{\prime}\right)}(\mathbf{u})$ for $1 \leq r, r^{\prime} \leq a(l)$.

4) $\ell_{j(l, r)}(\mathbf{u})<\ell_{j\left(l^{\prime}, r^{\prime}\right)}(\mathbf{u})$ if $l<l^{\prime}$.

We put

$$
S_{l}=\ell_{j(l, r)}(\mathbf{u})
$$

This is independent of $r$. Set

$$
\vec{v}_{l, r}=\vec{v}_{j(l, r)}=\left(v_{j(l, r), 1}, \ldots, v_{j(l, r), n}\right) \in \mathbb{Z}^{n} .
$$

It is an element of the dual vector space of $\mathbb{A}(\mathbb{Q})=\mathbb{Q}^{n}$, which we denote by $\mathbb{A}(\mathbb{Q})^{*}$. Here $\mathbb{A}(\mathbb{R})=\mathbb{A}(\mathbb{Q}) \otimes \mathbb{R}$ is the $\mathbb{R}$ vector space associated to the affine space which contains the moment polytope $P$. Let $\mathbb{A}_{l}^{\perp}$ is a vector subspace of $\mathbb{A}(\mathbb{Q})^{*}$ generated by $\left\{\vec{v}_{l^{\prime}, r} \mid l^{\prime} \leq l, r=1, \ldots, a\left(l^{\prime}\right)\right\}$. We denote by $K \leq K_{0}$ the smallest integer such that $\mathbb{A}_{K}^{\perp}=\mathbb{A}(\mathbb{Q})^{*}$. We have a filtration

$$
0 \subset \mathbb{A}_{1}^{\perp} \subset \mathbb{A}_{2}^{\perp} \subset \cdots \subset \mathbb{A}_{K}^{\perp}=\mathbb{A}(\mathbb{Q})^{*} .
$$

We put

$$
d(l)=\operatorname{dim} \mathbb{A}_{l}^{\perp}-\operatorname{dim} \mathbb{A}_{l-1}^{\perp}
$$

We have

$$
d(1)+\cdots+d(K)=n=\operatorname{dim} \mathbb{A}(\mathbb{Q})^{*} .
$$

Note $\mathbb{A} \cong \mathbb{Z}^{n} \subset \mathbb{A}(\mathbb{Q})=\mathbb{Q}^{n}$. So $\mathbb{Z}^{n} \subset \mathbb{A}(\mathbb{Q})^{*}$ is determined canonically. (We remark that $\mathbb{Z}^{n} \subset \mathbb{A}(\mathbb{Q})^{*}$ is generated by $\vec{v}_{j}, j=1, \ldots, m$.) Let $\left\{\mathbf{e}_{i}^{*} \mid i=\right.$ $1, \ldots, n\}$ be the standard basis of $\mathbb{Z}^{n} \subset \mathbb{A}(\mathbb{Q})^{*}$. We take $\mathbf{e}_{l, s}^{*}$ for $l=1, \ldots, K$, $s=1, \ldots, d(l)$ satisfying the following conditions.

Conditions 9.4. $\quad$ 1) $\left\{\mathbf{e}_{l^{\prime}, s}^{*} \mid l^{\prime} \leq l, s=1, \ldots, d\left(l^{\prime}\right)\right\}$ is a $\mathbb{Q}$ basis of $\mathbb{A}_{l}^{\perp}$.

2) $\vec{v}_{l, r}$ is contained in the $\mathbb{Z}$ module generated by $\left\{\mathbf{e}_{l^{\prime}, s}^{*} \mid l^{\prime} \leq l, s=\right.$ $\left.1, \ldots, d\left(l^{\prime}\right)\right\}$.

We define $b_{l^{\prime}, s ; i} \in \mathbb{Q}$ by

$$
\mathbf{e}_{l^{\prime}, s}^{*}=\sum_{i=1}^{n} b_{l^{\prime}, s ; i} \mathbf{e}_{i}^{*}
$$

and put

$$
\bar{y}_{l^{\prime}, s}=\prod_{i=1}^{n} \bar{y}_{i}^{b_{l^{\prime}, s ; i}}=\exp \left(\sum_{i=1}^{n} b_{l^{\prime}, s ; i} x_{i}\right) .
$$


(Note $\bar{y}_{i}=e^{x_{i}}$.) Since $b_{l^{\prime}, s ; i}$ may not be an integer, $\bar{y}_{l^{\prime}, s}$ may not be contained in the Laurent polynomial ring $\Lambda_{0}\left[\bar{y}, \bar{y}^{-1}\right]$ of the variables $\bar{y}_{i}(i=1, \ldots, n)$. But it is contained in the finite extention of it.

By Condition 9.4 2),

$$
z_{j(l, r)}=T^{S_{l}} \bar{y}_{1}^{v_{j(l, r), 1}} \cdots \bar{y}_{n}^{v_{j(l, r), n}}
$$

is contained in $\Lambda_{0}\left[\bar{y}, \bar{y}^{-1}\right]$. Moreover it is contained in the Laurent polynomial ring of the variables $\bar{y}_{l^{\prime}, s}, l^{\prime}=1, \ldots, l, s=1, \ldots, d\left(l^{\prime}\right)$.

We define $c_{l, r ; l^{\prime}, s} \in \mathbb{Z}$ by

$$
z_{j(l, r)}=T^{S_{l}} \prod_{l^{\prime} \leq l} \prod_{s \leq d\left(l^{\prime}\right)} \bar{y}_{l^{\prime}, s}^{c_{l, r} l^{\prime}, s} .
$$

In other words

$$
\vec{v}_{l, r}=\sum_{l^{\prime} \leq l} \sum_{s \leq d\left(l^{\prime}\right)} c_{l, r ; l^{\prime}, s} e_{l^{\prime}, s}^{*}
$$

We put

$$
\left(\mathfrak{P O}_{0}^{\mathfrak{b}}\right)_{l}=T^{-S_{\ell}} \sum_{r=1}^{a(l)} \mathfrak{c}_{j(l, r)} z_{j(l, r)}=\sum_{r=1}^{a(l)} \mathfrak{c}_{j(l, r)} \prod_{l^{\prime} \leq l} \prod_{s \leq d\left(l^{\prime}\right)} \bar{y}_{l^{\prime}, s}^{c_{l, r}, l^{\prime}, s} .
$$

The numbers $\mathfrak{c}_{j(l, r)} \in\left\{c \in \Lambda_{0} \mid \mathfrak{v}_{T}(c)=0\right\}$ are defined in Definition 9.1.

We remark $\left(\mathfrak{P O}_{0}^{\mathfrak{b}}\right)_{l}$ is a Laurent polynomial of variables $\bar{y}_{l^{\prime}, s}, l^{\prime} \leq l, s=$ $1, \ldots, d\left(l^{\prime}\right)$ with coefficients in a field of complex numbers.

Definition 9.5. The leading term equation is a system of $n$ equations of $n$ variables $\bar{y}_{l, s}$ with complex number coefficients. We define it by

$$
\left\{\begin{array}{cc}
\bar{y}_{1, s} \frac{\partial\left(\mathfrak{P} \mathfrak{O}_{0}^{\mathfrak{b}}\right)_{1}}{\partial \bar{y}_{1, s}}=0 & s=1, \ldots, d(1), \\
\bar{y}_{2, s} \frac{\partial\left(\mathfrak{P} \mathfrak{O}_{0}^{\mathfrak{b}}\right)_{2}}{\partial \bar{y}_{2, s}}=0 & s=1, \ldots, d(2), \\
\ldots & \\
\bar{y}_{l, s} \frac{\partial\left(\mathfrak{P O}_{0}^{\mathfrak{b}}\right)_{l}}{\partial \bar{y}_{l, s}}=0 & s=1, \ldots, d(l), \\
\ldots & \\
\bar{y}_{K, s} \frac{\partial\left(\mathfrak{P O}_{0}^{\mathfrak{b}}\right)_{K}}{\partial \bar{y}_{K, s}}=0 & s=1, \ldots, d(K) .
\end{array}\right.
$$

Note the first equation in (61) contains $\bar{y}_{1, s} s=1, \ldots, d(1)$, the second equation in (61) contains $\bar{y}_{1, s} s=1, \ldots, d(1)$ and $\bar{y}_{2, s} s=1, \ldots, d(2)$ etc.

If $\mathfrak{b}-\mathfrak{b}^{\prime} \in \mathcal{A}^{2}\left(\Lambda_{+}\right) \oplus \bigoplus_{k \neq 2} \mathcal{A}^{k}\left(\Lambda_{0}\right)$ then $\left(\mathfrak{P O} \mathfrak{O}_{0}^{\mathfrak{b}}\right)_{l}=\left(\mathfrak{P O}_{0}^{\mathfrak{b}^{\prime}}\right)_{l}$. So the leading term equation is the same for such $\mathfrak{b}$ and $\mathfrak{b}^{\prime}$. 
One of the main results of $[$ FOOO4] is as follows.

Theorem 9.6. Let $\mathbf{u} \in \operatorname{Int} P$ and $\mathfrak{b} \in \mathcal{A}\left(\Lambda_{0}\right)$. Then the following two conditions are equivalent.

1) The leading term equation (61) has a solution $\bar{y}_{l, s} \in \mathbb{C} \backslash\{0\}$.

2) There exists $b \in H^{1}\left(L(\mathbf{u}) ; \Lambda_{0}\right)$ and $\mathfrak{b}^{\prime} \in \mathcal{A}\left(\Lambda_{0}\right)$ with $\mathfrak{b}-\mathfrak{b}^{\prime} \in \mathcal{A}^{2}\left(\Lambda_{+}\right)$ such that

$$
H F\left((L(\mathbf{u}),(\mathfrak{b}, b)),(L(\mathbf{u}),(\mathfrak{b}, b)) ; \Lambda_{0}\right) \cong H\left(T^{n} ; \Lambda_{0}\right) .
$$

This is [FOOO4] Theorem 4.5 and Proposition 11.3. We omit the proof and refer [FOOO4].

DeFinition 9.7. We say that $L(\mathbf{u})$ is strongly bulk balanced if there exists $\mathfrak{b} \in \mathcal{A}\left(\Lambda_{0}\right)$ and $b \in H^{1}\left(L(\mathbf{u}) ; \Lambda_{0}\right)$ such that

$$
H F\left((L(\mathbf{u}),(\mathfrak{b}, b)),(L(\mathbf{u}),(\mathfrak{b}, b)) ; \Lambda_{0}\right) \cong H\left(T^{n} ; \Lambda_{0}\right) .
$$

See [FOOO4] Definition 3.13 for a related definition.

Theorem 9.6 gives a way to locate strongly bulk balanced $L(\mathbf{u})$ in terms of the leading term equation.

\section{Examples 2}

EXAMPLE 10.1. We consider Hirzebruch surface $F_{n}, n \geq 2$. We take its Kähler form so that the moment polytope is

$$
P=\left\{\left(u_{1}, u_{2}\right) \mid 0 \leq u_{1}, u_{2}, u_{1}+n u_{2} \leq n, u_{2} \leq 1-\alpha\right\},
$$

$0<\alpha<1$. The leading order potential function is

$$
\mathfrak{P O _ { 0 }}=y_{1}+y_{2}+T^{n} y_{1}^{-1} y_{2}^{-n}+T^{1-\alpha} y_{2}^{-1} \text {. }
$$

We put

$$
\begin{aligned}
& \ell_{1}\left(u_{1}, u_{2}\right)=u_{1}, \quad \ell_{2}\left(u_{1}, u_{2}\right)=u_{2}, \\
& \ell_{3}\left(u_{1}, u_{2}\right)=n-u_{1}-n u_{2}, \quad \ell_{4}\left(u_{1}, u_{2}\right)=1-\alpha-u_{2} .
\end{aligned}
$$

We put $S_{1}\left(u_{1}, u_{2}\right)=\inf \left\{\ell_{j}\left(u_{1}, u_{2}\right) \mid j=1,2,3,4\right\}$.

Suppose the first of the leading term equation (61) has a nonzero solution. Then it is easy to see that $d(1) \geq 2$. Namely

$$
\#\left\{j \mid S_{1}\left(u_{1}, u_{2}\right)=\ell_{j}\left(u_{1}, u_{2}\right)\right\} \geq 2 .
$$

This is satisfied on the 5 line seguments $l_{1}, \ldots, l_{5}$, where

$$
\begin{aligned}
& l_{1}: u_{1}=u_{2} \leq(1-\alpha) / 2, \quad l_{2}: u_{1}=1-\alpha-u_{2} \leq(1-\alpha) / 2, \\
& l_{3}: u_{1}=n-(n+1) u_{2} \geq n-(n+1)(1-\alpha) / 2 \\
& l_{4}: u_{1}=n-1+\alpha-(n-1) u_{2} \geq n-(n-1)(1-\alpha) / 2, \\
& l_{5}: u_{2}=(1-\alpha) / 2,(1-\alpha) / 2 \leq u_{1} \leq n-(n-1)(1-\alpha) / 2 .
\end{aligned}
$$




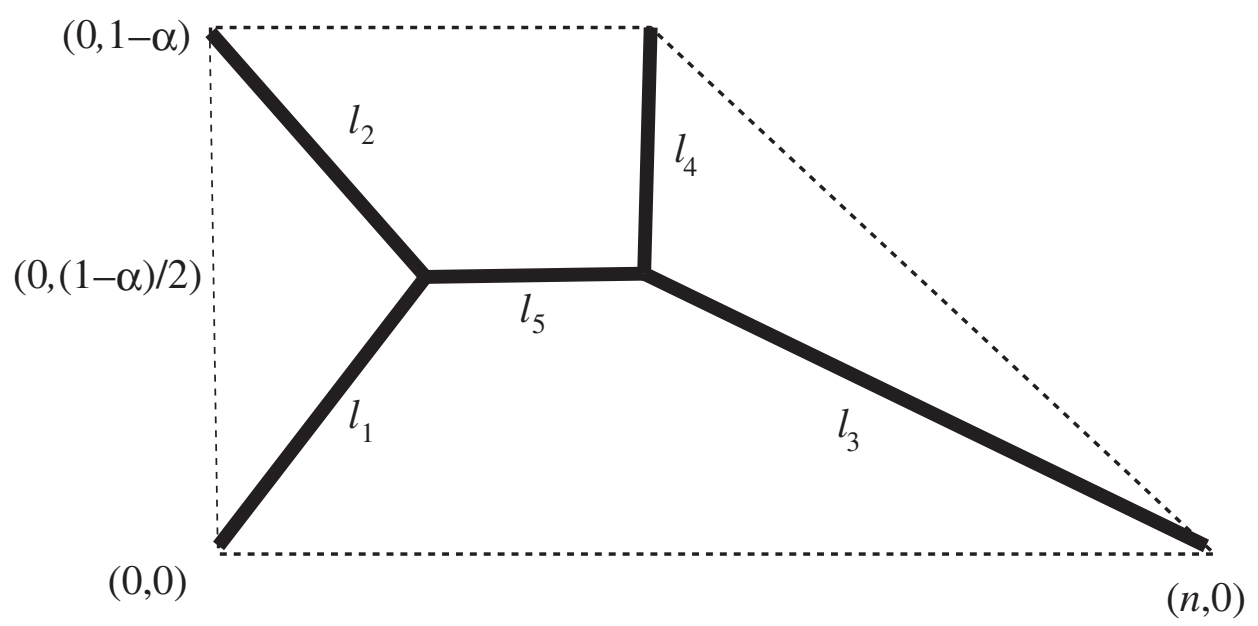

FiguRE 10.1

Note

$$
v_{1}=(1,0), v_{2}=(0,1), v_{3}=(-1,-n), v_{4}=(0,-1) .
$$

Let $\mathbf{u}=\left(u_{1}, u_{2}\right) \in l_{5}$. Then $\mathbb{A}_{1}^{\perp}$ is $\mathbb{Q} \cdot(0,1)$ and

$$
\left(\mathfrak{P O}_{0}^{\mathbf{u}}\right)_{1}=\bar{y}_{2}+\bar{y}_{2}^{-1}
$$

$\left(\right.$ Here $\mathfrak{b}=0$ and so we do not write $\mathfrak{b}$ in the above notation. We put $\bar{y}_{i}=y_{i}^{\mathbf{u}}$.)

We also have

$$
\left(\mathfrak{P O}_{0}^{\mathbf{u}}\right)_{2}= \begin{cases}\bar{y}_{1} & \text { If } u_{1}<(1+\alpha) n / 4 \\ \bar{y}_{1}^{-1} \bar{y}_{2}^{-n} & \text { If } u_{1}>(1+\alpha) n / 4 \\ \bar{y}_{1}+\bar{y}_{1}^{-1} \bar{y}_{2}^{-n} & \text { If } u_{1}=(1+\alpha) n / 4\end{cases}
$$

(62) gives the first leading term equation $1-\bar{y}_{2}^{-2}=0$ whose solutions are $\bar{y}_{2}= \pm 1$.

Then (63) gives the second of the leading term equation which are $1=0$, $-( \pm 1)^{-n} \bar{y}_{1}^{-2}=0,1-( \pm 1)^{-n} \bar{y}_{1}^{-2}=0$, where $u_{1}<(1+\alpha) n / 4, u_{1}>(1+\alpha) n / 4$ and $u_{1}=(1+\alpha) n / 4$, respectively.

The solution $\bar{y}_{1} \neq 0$ exists only in the case $u_{1}=(1+\alpha) n / 4$. In that case the solutions of leading term equations are $(1, \pm 1)$ and $\left(-1, \pm(-1)^{n / 2}\right)$. Thus $L((1+\alpha) n / 4,(1-\alpha) / 2)$ is strongly bulk balanced.

We can check that there are no other strongly bulk balanced $T^{2}$ orbits. (This follows from Theorem 11.9 also.)

See [FOOO3] Example 8.2 where the same conclusion is proved by basically the same but a slightly different calculation.

REMARK 10.2. For the case of Example 10.1 we can actually prove that $L((1+\alpha) n / 4,(1-\alpha) / 2)$ is strongly balanced. Namely some Floer cohomology 
without bulk deformation is non-zero. This follows from [FOOO3] Theorem 10.4 .

EXAmple 10.3. ([FOOO4] section 5, [FOOO3] Example 10.17.) We consider two points blow up $X\left(\alpha, \alpha^{\prime}\right)$ of $\mathbb{C} P^{2}$. (Example 4.4.) We consider the case $\alpha>1 / 3, \alpha^{\prime}=(1-\alpha) / 2$. The moment polytope is

$$
P=\left\{\left(u_{1}, u_{2}\right) \mid 0 \leq u_{1} \leq 1,0 \leq u_{2} \leq 1-\alpha,(1-\alpha) / 2 \leq u_{1}+u_{2} \leq 1\right\} .
$$

We consider

$$
\mathbf{u}(t)=(t,(1-\alpha) / 2), \quad t \in((1-\alpha) / 2,(1+\alpha) / 4) .
$$

We have

$$
\mathfrak{P O}=T^{(1-\alpha) / 2}\left(\bar{y}_{2}+\bar{y}_{2}^{-1}\right)+T^{t}\left(\bar{y}_{1}+\bar{y}_{1} \bar{y}_{2}\right)+T^{(1+\alpha) / 2+t}\left(\bar{y}_{1} \bar{y}_{2}\right)^{-1}
$$

where

Therefore

$$
(1-\alpha) / 2<t<(1+\alpha) / 2+t
$$

$$
(\mathfrak{P O})_{1}=\bar{y}_{2}+\bar{y}_{2}^{-1}, \quad(\mathfrak{P O})_{2}=\bar{y}_{1}+\bar{y}_{1} \bar{y}_{2} .
$$

Thus the leading term equation is

$$
1-\bar{y}_{2}^{-2}=0, \quad 1+\bar{y}_{2}=0 .
$$

This has a solution $\bar{y}_{2}=-1\left(\bar{y}_{1}\right.$ is any number $\in \mathbb{C} \backslash\{0\}$.)

Theorem 9.6 implies that all of $L(\mathbf{u}(t))$ as in (64) are strongly bulk balanced. In particular they are non-displaceable.

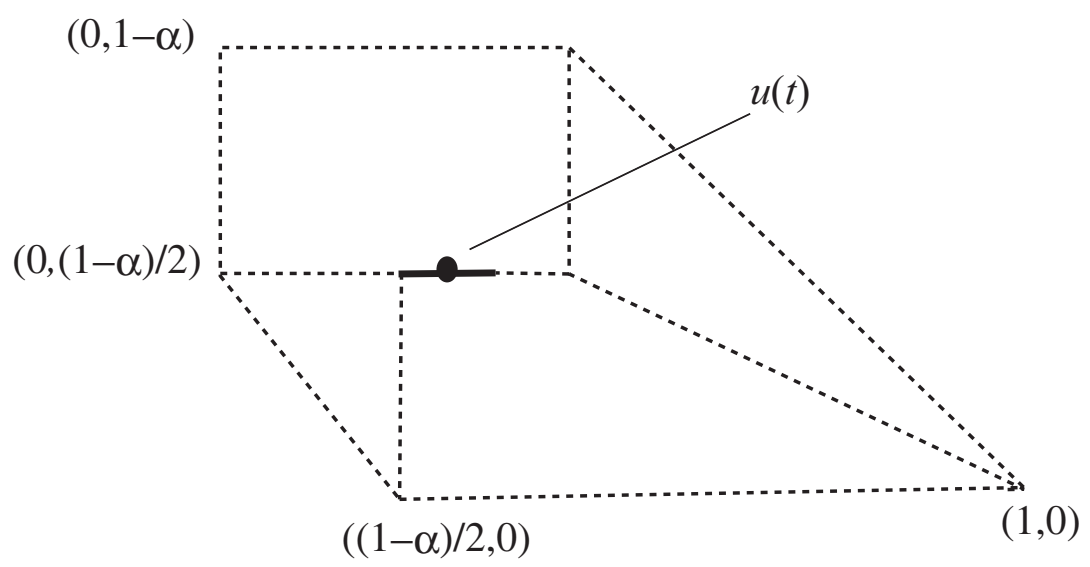

FiguRE 10.2

REMARK 10.4. In the toric case, for each given $\mathfrak{b}$, the number of $L(\mathbf{u})$ with nontrivial Floer cohomology for a pair $(\mathfrak{b}, b)$ for some $b \in H^{1}\left(L(\mathbf{u}) ; \Lambda_{0}\right)$ is finite. (It is not greater than the Betti number of $X$ by Theorem 11.9.) So to obtain infinitely many $L(\mathbf{u})$ with nontrivial Floer cohomology we need to include bulk deformations. 
In the examples we discussed in this section, we do not need to change the variables from $y_{j}$ to $y_{l, s}$. An example where we need this change of variables is given in [FOOO3] Example 10.10.

In Example 10.3 we obtain a continuum of non-displaceable Lagrangian torus in certain two points blow up of $\mathbb{C} P^{2}$. ([FOOO4]). We can also use bulk deformation to obtain a continuum of Lagrangian tori in $S^{2} \times S^{2}$. They are not of the type of $T^{2}$ orbit but is obtained from the $T^{2}$ orbit of singular Hirzebruch surface $F_{2}(0)$ by deforming the singularity, that is of orbifold of $A_{1}$-type. ([FOOO6].) Closely related construction is in [NNU1, NNU2]

\section{Quantum cohomology and Jacobian ring}

11.1. Jacobian ring over Novikov ring. In this section we discuss the isomorphism between the Jacobian ring of $\mathfrak{P O}^{\mathfrak{b}}$ and the quantum cohomology ring of $X$ deformed by $\mathfrak{b}$. We start with defining Jacobian ring precisely.

Usually Jacobian ring is studied in the case of (Laurent) polynomial or holomorphic function germ. Our function $\mathfrak{P O}^{\mathfrak{b}}$ is neither a Laurent polynomial and nor a holomorphic function. So we first define a function space in which $\mathfrak{P O}^{\mathfrak{b}}$ is contained.

We consider the Laurent polynomial ring $\Lambda\left[y, y^{-1}\right]$ of $n$ variables with $\Lambda$ coefficients. We defined a valuation $\mathfrak{v}_{T}^{\mathbf{u}}$ for each $\mathbf{u} \in \mathbb{R}^{n}$ in section 5 Definition 5.6. Let $P$ be a compact subset of $\mathbb{R}^{n}$. (We use the case when $P$ is a convex polytope only in this article.)

Definition 11.1. For $F \in \Lambda\left[y, y^{-1}\right]$ we define

$$
\mathfrak{v}_{T}^{P}(F)=\inf \left\{\mathfrak{v}_{T}^{\mathbf{u}}(F) \mid \mathbf{u} \in P\right\} .
$$

This is not a valuation but is a norm. Therefore it defines a metric on $\Lambda\left[y, y^{-1}\right]$ by $d_{P}\left(F_{1}, F_{2}\right)=e^{-\mathfrak{v}_{T}^{P}\left(F_{1}-F_{2}\right)}$. We denote the completion of $\Lambda\left[y, y^{-1}\right]$ with respect to $d_{P}$ by $\Lambda\left\langle\left\langle y, y^{-1}\right\rangle\right\rangle^{P}$. It is a normed ring.

We define $\Lambda\left\langle\left\langle y, y^{-1}\right\rangle\right\rangle_{0}^{P}$ as the set of all $F \in \Lambda\left\langle\left\langle y, y^{-1}\right\rangle\right\rangle^{P}$ such that $\mathfrak{v}_{T}^{P}(F) \geq 0$.

Let $P$ be a moment polytope of our toric manifold $X$. We take $\ell_{j}(j=$ $1, \ldots, m)$ as in Condition 4.1 and put

$$
P_{\epsilon}=\left\{\mathbf{u} \in \mathbb{R}^{n} \mid \ell_{j}(\mathbf{u}) \geq \epsilon, j=1, \ldots, m\right\}
$$

for $\epsilon>0$. We define $\mathfrak{v}_{T}^{P_{\epsilon}}, \Lambda\left\langle\left\langle y, y^{-1}\right\rangle\right\rangle^{P_{\epsilon}}$ and $\Lambda\left\langle\left\langle y, y^{-1}\right\rangle\right\rangle_{0}^{P_{\epsilon}}$ in a similar way.

Definition 11.2. We define a metric $d_{P}$ on $\Lambda\left[y, y^{-1}\right]$ by

$$
d_{\stackrel{\circ}{\circ}}\left(F_{1}, F_{2}\right)=\sum_{k=1}^{\infty} 2^{-k} \min \left(d_{P_{1 / k}}\left(F_{1}, F_{2}\right), 1\right) .
$$

Let $\Lambda\left\langle\left\langle y, y^{-1}\right\rangle\right\rangle^{P}$ be the completion of $\Lambda\left[y, y^{-1}\right]$ with respect to $d_{P}$ and define $\Lambda\left\langle\left\langle y, y^{-1}\right\rangle\right\rangle_{0}^{\stackrel{\circ}{P}}=\bigcap_{k=1}^{\infty} \Lambda\left\langle\left\langle y, y^{-1}\right\rangle\right\rangle_{0}^{P_{1 / k}}$. 
It is easy to see that an element of $\Lambda\left\langle\left\langle y, y^{-1}\right\rangle\right\rangle^{P}$ may be regarded as a function $: \mathfrak{A}(P) \rightarrow \Lambda$ and an element of $\Lambda\left\langle\left\langle y, y^{-1}\right\rangle\right\rangle^{P}$ may be regarded as a function $: \mathfrak{A}(\stackrel{\circ}{P}) \rightarrow \Lambda$.

LEMma 11.3. If $\mathfrak{b} \in \mathcal{A}\left(\Lambda_{0}\right)$ then

$$
\mathfrak{P O}^{\mathfrak{b}} \in \Lambda\left\langle\left\langle y, y^{-1}\right\rangle\right\rangle_{0}^{\stackrel{\circ}{P}}, \quad y_{i} \frac{\partial \mathfrak{P \mathcal { O } ^ { \mathfrak { b } }}}{\partial y_{i}} \in \Lambda\left\langle\left\langle y, y^{-1}\right\rangle\right\rangle_{0}^{\stackrel{\circ}{P}}
$$

If $\mathfrak{b} \in \mathcal{A}\left(\Lambda_{+}\right)$then

$$
\mathfrak{P O}^{\mathfrak{b}} \in \Lambda\left\langle\left\langle y, y^{-1}\right\rangle\right\rangle_{0}^{P}, \quad y_{i} \frac{\partial \mathfrak{P} \mathfrak{D}^{\mathfrak{b}}}{\partial y_{i}} \in \Lambda\left\langle\left\langle y, y^{-1}\right\rangle\right\rangle_{0}^{P}
$$

We omit the proof, which follows from Theorem 8.2. See [FOOO5] Lemma 2.6. Now we define

DEFINITION 11.4.

$$
\operatorname{Jac}\left(\mathfrak{P O}^{\mathfrak{b}}\right)=\frac{\Lambda\left\langle\left\langle y, y^{-1}\right\rangle\right\rangle_{0}^{P}}{\operatorname{Clos}_{d_{P}}\left(y_{i} \frac{\partial \mathfrak{P} \mathfrak{O}^{\mathfrak{b}}}{\partial y_{i}}: i=1, \ldots, n\right)} .
$$

(We may replace $\Lambda\left\langle\left\langle y, y^{-1}\right\rangle\right\rangle_{0}^{P}$ by $\Lambda\left\langle\left\langle y, y^{-1}\right\rangle\right\rangle_{0}^{P}$ in the above formula in case $\mathfrak{b} \in \mathcal{A}\left(\Lambda_{+}\right)$.)

Here the denominator is the closure of the ideal generated by $y_{i} \frac{\partial \mathfrak{P O ^ { \mathfrak { b } }}}{\partial y_{i}}$ : $i=1, \ldots, n$. The closure is taken with respect to the metric $d_{P}$.

11.2. Big quantum cohomology: a quick review. We next review briefly the well established story of deformed quantum cup product. Let $(X, \omega)$ be a symplectic manifold. For $\alpha \in H_{2}(X ; \mathbb{Z})$ let $\mathcal{M}_{\ell}(\alpha)$ be the moduli space of stable maps from genus zero semi-stable curves with $\ell$ marked points and of homology class $\alpha$. There exists an evaluation map

$$
\text { ev }: \mathcal{M}_{\ell}(\alpha) \rightarrow X^{\ell}
$$

$\mathcal{M}_{\ell}(\alpha)$ has a virtual fundamental cycle and hence defines a class

$$
\mathrm{ev}_{*}\left[\mathcal{M}_{\ell}(\alpha)\right] \in H_{*}\left(X^{\ell} ; \mathbb{Q}\right) .
$$

(See $[\mathbf{F O}]$.$) Here *=2 n+2 c_{1}(X) \cap \alpha+2 \ell-6$. Let $Q_{1}, \ldots, Q_{\ell}$ be cycles such that

$$
\sum \operatorname{codim} Q_{i}=2 n+2 c_{1}(X) \cap \alpha+2 \ell-6 .
$$

We define Gromov-Witten invariant by

$$
G W_{\ell}\left(\alpha: Q_{1}, \ldots, Q_{\ell}\right)=\operatorname{ev}_{*}\left[\mathcal{M}_{\ell}(\alpha)\right] \cap\left(Q_{1} \times \cdots \times Q_{\ell}\right) \in \mathbb{Q} .
$$

We put $G W_{\ell}\left(\alpha: Q_{1}, \ldots, Q_{\ell}\right)=0$ when $(67)$ is not satisfied. 
We now define

$$
G W_{\ell}\left(Q_{1}, \ldots, Q_{\ell}\right)=\sum_{\alpha} T^{(\alpha \cap \omega) / 2 \pi} G W_{\ell}\left(\alpha: Q_{1}, \ldots, Q_{\ell}\right)
$$

The formula (68) extends to a $\Lambda_{0}$ module homomorphism

$$
G W_{\ell}: H\left(X ; \Lambda_{0}\right)^{\otimes \ell} \rightarrow \Lambda_{0}
$$

Definition 11.5. Let $\mathfrak{b} \in H\left(X ; \Lambda_{0}\right)$ be given. For each given pair $\mathfrak{c}, \mathfrak{d} \in$ $H\left(X ; \Lambda_{0}\right)$, we define a product $\mathfrak{c} \cup^{\mathfrak{b}} \mathfrak{d} \in H\left(X ; \Lambda_{0}\right)$ by the following formula

$$
\left\langle\mathfrak{c} \cup^{\mathfrak{b}} \mathfrak{d}, \mathfrak{e}\right\rangle_{\mathrm{PD}_{X}}=\sum_{\ell=0}^{\infty} \frac{1}{\ell !} G W_{\ell+3}(\mathfrak{c}, \mathfrak{d}, \mathfrak{e}, \mathfrak{b}, \ldots, \mathfrak{b}) .
$$

Here $\langle\cdot, \cdot\rangle_{\mathrm{PD}_{X}}$ denotes the Poincaré duality pairing. The right hand side converges if $\mathfrak{b} \in H^{2}\left(X ; \Lambda_{+}\right) \oplus \bigoplus_{k>2} H^{k}\left(X ; \Lambda_{0}\right)$. We can extend it to arbitrary $\mathfrak{b} \in H^{*}\left(X ; \Lambda_{0}\right)$. (This is well-known. See for example [FOOO5] section 2.)

$\cup^{\mathfrak{b}}$ defines a graded commutative and associative ring structure on $H\left(X ; \Lambda_{0}\right)$. We call $\cup^{\mathfrak{b}}$ the deformed quantum cup product.

\subsection{The isomorphism 'Jacobian ring = quantum cohomology' and its applications.}

THEOREM 11.6. There exists a ring isomorphism

$$
\left(H\left(X ; \Lambda_{0}\right), \cup^{\mathfrak{b}}\right) \cong \operatorname{Jac}\left(\mathfrak{P} \mathfrak{O}^{\mathfrak{b}}\right) .
$$

This is [FOOO5] Theorem 1.1.1 (1). We explain some parts of the proof later in this section. We first discuss some applications.

Definition 11.7. Let $\operatorname{Crit}\left(\mathfrak{P O}^{\mathfrak{b}}\right)$ be the set of all $\mathfrak{y} \in \mathfrak{A}(\stackrel{\circ}{P})$ such that

$$
\frac{\partial \mathfrak{P \mathfrak { O } ^ { \mathfrak { b } }}}{\partial y_{i}}(\mathfrak{y})=0
$$

for $i=1, \ldots, n$. An element of $\operatorname{Crit}\left(\mathfrak{P O}^{\mathfrak{b}}\right)$ is said to be a critical point of $\mathfrak{P} \mathfrak{O}^{\mathfrak{b}}$.

A critical point $\mathfrak{y}$ of $\mathfrak{P O}^{\mathfrak{b}}$ is said to be non-degenerate if the matrix

$$
\left[\mathfrak{y}_{i} \mathfrak{y}_{j} \frac{\partial^{2} \mathfrak{P} \mathfrak{O}^{\mathfrak{b}}}{\partial y_{i} \partial y_{j}}(\mathfrak{y})\right]_{i, j=1}^{i, j=n}
$$

is invertible, as an $n \times n$ matrix with $\Lambda$ entries.

The function $\mathfrak{P O}^{\mathfrak{b}}$ is said to be a Morse function if all of its critical points are non-degenerate. 
We put

$$
\mathfrak{M}(X ; \mathfrak{b})=\left\{\begin{array}{l|l}
(\mathbf{u}, b) & \begin{array}{l}
\mathbf{u} \in \operatorname{Int} P, b \in H^{1}\left(L(\mathbf{u}) ; \Lambda_{0}\right) / H^{1}(L(\mathbf{u}) ; 2 \pi \sqrt{-1} \mathbb{Z}), \\
H F\left((L(\mathbf{u}),(\mathfrak{b}, b)),(L(\mathbf{u}),(\mathfrak{b}, b)) ; \Lambda_{0}\right) \cong H\left(T^{n} ; \Lambda_{0}\right)
\end{array}
\end{array}\right\}
$$

Theorem 8.6 implies the following.

$$
\# \mathfrak{M}(X ; \mathfrak{b})=\# \operatorname{Crit}\left(\mathfrak{P O}^{\mathfrak{b}}\right) \text {. }
$$

Proposition 11.8. There exists a direct product decomposition

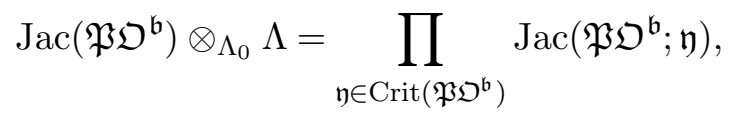

as a ring.

The factor $\operatorname{Jac}\left(\mathfrak{P O}^{\mathfrak{b}} ; \mathfrak{y}\right)$ in the right hand side is a local ring.

The ring $\operatorname{Jac}\left(\mathfrak{P O}^{\mathfrak{b}} ; \mathfrak{y}\right)$ is one dimensional if and only if $\mathfrak{y}$ is nondegenerate.

This is a standard result in the case, for example, when the function $\left(\mathfrak{P \mathfrak { O } ^ { \mathfrak { b } }}\right.$ in our case) is a polynomial or a holomorphic function. We can prove Proposition 11.8 in a similar way to those cases. It is proved in [FOOO5] section 2.2 .

Theorem 11.6 and Proposition 11.8 imply that $\left(H(X ; \Lambda), \cup^{\mathfrak{b}}\right)$ is semisimple if and only if $\mathfrak{P O}^{\mathfrak{b}}$ is a Morse function.

Theorem 11.6 together with Proposition 11.8 and Formula (70) imply the following:

TheOREM 11.9. $\quad$ 1) If $\mathfrak{P O}^{\mathfrak{b}}$ is a Morse function then

$$
\operatorname{rank} H(X ; \mathbb{Q})=\# \mathfrak{M}(X ; \mathfrak{b}) .
$$

2) If $\mathfrak{P O}^{\mathfrak{b}}$ is not a Morse function then

$$
0<\# \mathfrak{M}(X ; \mathfrak{b})<\operatorname{rank} H(X ; \mathbb{Q}) .
$$

This is [FOOO5] Theorem 1.1.3. Some of the earlier partial results is given in [FOOO3] Theorems 1.9 and 1.12.

REMARK 11.10. Theorem 11.9 in particular implies that there exists at least one non-displaceable $T^{n}$ orbit. This fact also follows from an earlier result by Entov-Polterovich [EP2, EP3].

Another application is the following:

TheOrem 11.11. ([FOOO5] Theorem 1.1.4.) Assume $\mathfrak{b} \in H^{2}\left(X ; \Lambda_{0}\right)$. The set of eigenvalues of the map $x \mapsto c_{1}(X) \cup^{\mathfrak{b}} x: H(X ; \Lambda) \rightarrow H(X ; \Lambda)$ coincides with the set of critical values of $\mathfrak{P O}^{\mathfrak{b}}$, with multiplicities counted.

Remark 11.12. Theorem 11.11 was conjectured by M. Kontsevich. See also [Aur1]. 
Proof. The proof uses the following:

LEMma 11.13. Let us consider the situation of Theorem 11.11. Then, by the isomorphism in Theorem 11.6, the first Chern class $c_{1}(X) \in H^{2}(X ; \mathbb{C})$ is sent to the equivalence class of $\mathfrak{P O}^{\mathfrak{b}}$ in $\operatorname{Jac}\left(\mathfrak{P O}^{\mathfrak{b}}\right)$.

This is [FOOO5] Proposition 2.12.1.

Now we consider $x \mapsto c_{1}(X) \cup^{\mathfrak{b}} x$. We use Thoerem 11.6 and Proposition 11.8 then it is identified to the direct sum of maps

$$
[F] \mapsto\left[\mathfrak{P O} \mathfrak{b}^{\mathfrak{b}} F\right], \quad \operatorname{Jac}\left(\mathfrak{P D ^ { \mathfrak { b } }} ; \mathfrak{y}\right) \rightarrow \operatorname{Jac}\left(\mathfrak{P O ^ { \mathfrak { b } }} ; \mathfrak{y}\right) .
$$

The eigenvalue of this map is $\mathfrak{P O}^{\mathfrak{b}}(\mathfrak{y})$. This implies Theorem 11.11.

11.4. Construction of the homomorphism $\mathfrak{k}_{\mathfrak{b}}$. In various applications of Thoerem 11.6 it is also important to know the way how the isomorphism is defined, which we describe in this subsection.

Let $\mathbf{p}_{i}$ be the basis of $\mathcal{A}$ as in section 8 . We write an element $\mathfrak{b} \in$ $\mathcal{A}\left(\Lambda_{0}\right)$ as

$$
\mathfrak{b}=\sum_{i=0}^{B} w_{i} \mathbf{p}_{i}
$$

We put $\mathfrak{w}_{i}=e^{w_{i}}$ for $i=1, \ldots, m$. (Note $\mathbf{p}_{i}, i=1, \ldots, m$ are degree 2 classes.) We define $P_{j_{0} \ldots j_{B}}(y)$ by

$$
\mathfrak{P O}(\mathfrak{b} ; y)=\sum_{j_{0}=0}^{\infty} \cdots \sum_{j_{B}=0}^{\infty} P_{j_{0} \ldots j_{B}}(y) w_{0}^{j_{0}} \mathfrak{w}_{1}^{j_{1}} \cdots \mathfrak{w}_{m}^{j_{m}} w_{m+1}^{j_{m+1}} \ldots w_{B}^{j_{B}} .
$$

We can show that

$$
P_{j_{0} \ldots j_{B}}(y) \in T^{\rho_{j_{0} \ldots j_{B}} \Lambda}\left\langle\left\langle y, y^{-1}\right\rangle\right\rangle_{0}^{P}
$$

with

$$
\lim _{j_{0}+\cdots+j_{B} \rightarrow \infty} \rho_{j_{0} \ldots j_{B}}=\infty .
$$

Therefore the right hand side of

$$
\begin{aligned}
\frac{\partial}{\partial w_{i}} \mathfrak{P O}(\mathfrak{b} ; y) \\
\quad= \begin{cases}\sum_{j_{0}=0}^{\infty} \cdots \sum_{j_{B}=0}^{\infty} j_{i} P_{j_{0} \ldots j_{B}}(y) w_{0}^{j_{0}} \cdots w_{i}^{j_{i}-1} \cdots w_{B}^{j_{B}} & i \neq 1, \ldots, m \\
\sum_{j_{0}=0}^{\infty} \cdots \sum_{j_{B}=0}^{\infty} j_{i} P_{j_{0} \ldots j_{B}}(y) w_{0}^{j_{0}} \cdots \mathfrak{w}_{i}^{j_{i}} \cdots w_{B}^{j_{B}} & i=1, \ldots, m\end{cases}
\end{aligned}
$$

makes sense and is contained in $\Lambda\left\langle\left\langle y, y^{-1}\right\rangle\right\rangle^{P}$ for each $\mathfrak{b} \in \mathcal{A}\left(\Lambda_{0}\right)$.

We define the map

$$
\tilde{\mathfrak{k}}_{\mathfrak{b}_{0}}: \mathcal{A}\left(\Lambda_{0}\right) \rightarrow \Lambda\left\langle\left\langle y, y^{-1}\right\rangle \stackrel{\circ}{P}_{0}\right.
$$


by

$$
\tilde{\mathfrak{k}_{\mathfrak{b}_{0}}}\left(\mathbf{p}_{i}\right)=\left.\frac{\partial}{\partial w_{i}} \mathfrak{P O}(\mathfrak{b} ; y)\right|_{\mathfrak{b}=\mathfrak{b}_{0}}
$$

THEOREM 11.14. There exists a $\Lambda_{0}$ module homomorphism $\mathfrak{k}_{\mathfrak{b}}$ such that the following diagram commutes:

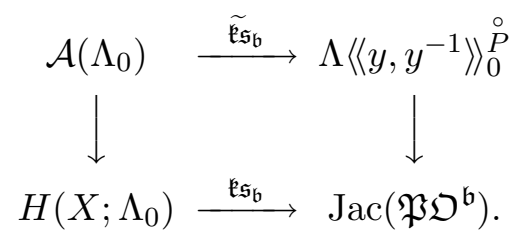

The map $\mathfrak{k}_{\mathfrak{b}}$ is the isomorphism mentioned in Theorem 11.6.

Theorem 11.14 is [FOOO5] Theorem 2.4.1.

Sketch of The PRoOf. By definition, we have

$$
\mathfrak{P O}(\mathfrak{b} ; y)=\sum_{k=0}^{\infty} \sum_{\ell=0}^{\infty} \int_{L(\mathbf{u})} \mathfrak{q}_{\ell ; k}\left(\mathfrak{b}^{\ell}, b^{k}\right)
$$

Here $b=\sum_{i=1}^{n} x_{i} \mathbf{e}_{i}$ and $y_{i}=e^{x_{i}}$. Using $\partial \mathfrak{b} / \partial w_{i}=\mathbf{p}_{i}$ we have

$$
\frac{\partial \mathfrak{P O}(\mathfrak{b} ; y)}{\partial w_{i}}=\sum_{k=0}^{\infty} \sum_{\ell_{1}=0}^{\infty} \sum_{\ell_{2}=0}^{\infty} \int_{L(\mathbf{u})} \mathfrak{q}_{\ell ; k}\left(\mathfrak{b}^{\ell_{1}} \mathbf{p}_{i} \mathfrak{b}^{\ell_{2}}, b^{k}\right)
$$

The homomorphism

$$
\mathbf{p}_{i} \mapsto \sum_{k=0}^{\infty} \sum_{\ell_{1}=0}^{\infty} \sum_{\ell_{2}=0}^{\infty} \mathfrak{q}_{\ell ; k}\left(\mathfrak{b}^{\ell_{1}} \mathbf{p}_{i} \mathfrak{b}^{\ell_{2}}, b^{k}\right)
$$

induces a homomorphism

$$
H\left(X ; \Lambda_{0}\right) \rightarrow H F\left((L(\mathbf{u}),(\mathfrak{b}, b)),(L(\mathbf{u}),(\mathfrak{b}, b)) ; \Lambda_{0}\right) .
$$

This fact was proved in [FOOO1] Theorem 3.8.62 for arbitrary $L \subset X$.

Note that to define (79) by (78) we fix $\mathfrak{b}, b$ and regard the right hand side of $(78)$ as an element of $H\left(L(\mathbf{u}), \Lambda_{0}\right)$. When we define $\tilde{\mathfrak{k}}_{\mathfrak{b}}$, we regard $b=\sum_{i=1}^{n} x_{i} \mathbf{e}_{i}$, as a $H\left(L(\mathbf{u}), \Lambda_{0}\right)$ valued function of $x_{i}$. So the right hand side of (77) is a function of $y_{i}=e^{x_{i}}$.

In other words we need to study the 'family version' of the welldefinedness of (79).

We consider the boundary operator

$$
a \in H\left(L(\mathbf{u}), \Lambda_{0}\right) \mapsto \mathfrak{m}_{1}^{\mathfrak{b}, b}(a)=\sum_{k_{1}=0}^{\infty} \sum_{k_{2}=0}^{\infty} \sum_{\ell=0}^{\infty} \mathfrak{q}_{\ell ; k}\left(\mathfrak{b}^{\ell}, b^{k_{1}} a b^{k_{2}}\right)
$$

The well-definedness of (79) means the following Claim 11.15. Let $i_{\mathrm{qm},(\mathfrak{b}, b)}^{*}$ $\left(\mathbf{p}_{i}\right)$ be the right hand side of $(78)$. 
ClaIm 11.15. If $\sum_{i=0}^{B} c_{i} \mathbf{p}_{i}$ is zero in $H\left(X ; \Lambda_{0}\right)$, then $\sum_{i=0}^{B} c_{i} i_{\mathrm{qm},(\mathfrak{b}, b)}^{*}\left(\mathbf{p}_{i}\right)$ lies in the image of $\mathfrak{m}_{1}^{\mathfrak{b}, b}$.

We can prove the same claim when we regard $b$ as a function of $x_{i}$. By the proof of Theorem 5.5 (especially by Formula (23)), the image of $\mathfrak{m}_{1}^{\mathfrak{b}, b}$ (where $b$ is regarded as a function of $x_{i}$ ) is in the Jacobian ideal (the ideal generated by $\left.y_{i} \partial \mathfrak{P O}^{\mathfrak{b}} / \partial y_{i}\right)$.

Thus the kernel of $\mathcal{A}\left(\Lambda_{0}\right) \rightarrow H\left(X ; \Lambda_{0}\right)$ is mapped to the Jacobian ideal by $\tilde{\mathfrak{k}}_{\mathfrak{b}}$. This implies the theorem.

Before closing this subsection, we state Theorem 11.17 which is a nonlinear version of Theorem 11.14.

The potential function with bulk $\mathfrak{P} \mathfrak{D}^{\mathfrak{b}}$ is parametrized by $\mathfrak{b} \in \mathcal{A}\left(\Lambda_{0}\right)$. Theorem 11.17 says that it depends only on the cohomology class $\mathfrak{b}$ up to appropriate change of variables. $\Lambda\left\langle\left\langle y, y^{-1}\right\rangle\right\rangle_{+}^{P}$ denotes the set of elements $R$ of $\Lambda\left\langle\left\langle y, y^{-1}\right\rangle \stackrel{\circ}{P}_{0}\right.$ such that $T^{-\epsilon} R \in \Lambda\left\langle\left\langle y, y^{-1}\right\rangle\right\rangle_{0}^{\stackrel{\circ}{P}}$ for some $\epsilon>0$.

Definition 11.16. We consider $n$ elements $y_{i}^{\prime} \in \Lambda\left\langle\left\langle y, y^{-1}\right\rangle\right\rangle^{P}$ $(i=1, \ldots, n)$.

1) We say that $y^{\prime}=\left(y_{1}^{\prime}, \ldots, y_{n}^{\prime}\right)$ is a coordinate change converging on Int $P$ (or a coordinate change on Int $P$ ) if

$$
y_{i}^{\prime} \equiv c_{i} y_{i} \bmod y_{i} \Lambda\left\langle\left\langle y, y^{-1}\right\rangle \stackrel{\circ}{P}_{+}\right.
$$

$c_{i} \in \mathbb{C} \backslash\{0\}$.

2) We say that the coordinate change is strict if $c_{i}=1$ for all $i$.

3) We say that the coordinate change converges on $P$ if $y_{i}^{\prime} \in$ $\Lambda\left\langle\left\langle y, y^{-1}\right\rangle\right\rangle^{P}(i=1, \ldots, n)$ in addition. Its strictness is defined in the same way. We also say that $y^{\prime}$ is a coordinate change on $P$.

The set of all coordinate changes forms a group. It is regarded as a kind of group of self automorphisms of the filtered $A_{\infty}$ algebra associated to $L(\mathbf{u})$. (The domain of convergence assumed in Definition 11.16 requires that it converges not only by the norm $\mathfrak{v}_{T}^{\mathbf{u}}$ but also by $\mathfrak{v}_{T}^{\mathbf{u}^{\prime}}$ with any $\mathbf{u}^{\prime}$. This is the reason we write "a kind of" in the above sentence.) A closely related group appears in [KS2] and [GPS].

Theorem 11.17. Let $\mathfrak{b}, \mathfrak{b}^{\prime} \in \mathcal{A}\left(\Lambda_{0}\right)$. We assume that $[\mathfrak{b}]=\left[\mathfrak{b}^{\prime}\right] \in$ $H\left(X ; \Lambda_{0}\right)$.

Then there exists a coordinate change $y^{\prime}$ on $\operatorname{Int} P$, such that

$$
\mathfrak{P O}^{\mathfrak{b}}\left(y^{\prime}\right)=\mathfrak{P O}^{\mathfrak{b}^{\prime}}(y) .
$$

If $\mathfrak{b}-\mathfrak{b}^{\prime} \in \mathcal{A}\left(\Lambda_{+}\right)$, then $y^{\prime}$ can be taken to be strict.

If both $\mathfrak{b}, \mathfrak{b}^{\prime} \in \mathcal{A}\left(\Lambda_{+}\right)$, then $y^{\prime}$ can be taken to be a strict coordinate change on $P$. 
This is [FOOO5] Theorem 8.7.

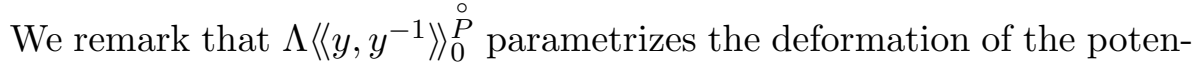
tial function. Then the Jacobian ideal corresponds to the part induced by the coordinate change. Thus Theorem 11.17 follows from Theorem 11.14 by some 'integration' (that is solving appropriate ordinary differential equation.) See [FOOO5] section 8.

11.5. The homomorphism $\mathfrak{k}_{\mathfrak{b}}$ is an isomorphism. The main geometric input to the proof of Theorem 11.6 is the following:

TheOREM 11.18. The map $\mathfrak{k}_{\mathfrak{b}}:\left(H\left(X ; \Lambda_{0}\right), \cup^{\mathfrak{b}}\right) \rightarrow \operatorname{Jac}\left(\mathfrak{P O}^{\mathfrak{b}}\right)$ is a ring homomorphism.

Theorem 11.18 is [FOOO5] Theorem 2.6.1.

Note this theorem is a version of a result which holds in greater generality. Namely there exists a ring homomorphism

$$
Q H\left(X ; \Lambda_{0}\right) \rightarrow H H(F u k(X, \omega)),
$$

where the right hand side is the Hochschild cohomology of the Fukaya category (see [Fu1, FOOO8] for its definition.) The existence of such homomorphism was first suggested by $[\mathbf{K o}]$ and conjectured explicitly by $[\mathbf{S e 3}]$ etc. See [FOOO5] section 4.5 and the reference therein for some of the related works.

We remark that $H H(F u k(X, \omega))$ parametrizes the deformation of the Lagrangian Floer theory on $X$. The Jacobian $\operatorname{ring} \operatorname{Jac}\left(\mathfrak{P O}^{\mathfrak{b}}\right)$ parametrizes the deformation of a part of the structures, that is the part described by $\mathfrak{m}_{0}^{b}(1)$. So there is a natural ring homomorphism $H H(F u k(X, \omega)) \rightarrow$ $\operatorname{Jac}\left(\mathfrak{P} \mathfrak{O}^{\mathfrak{b}}\right)$ in the toric case. Combining them we obtain the ring homomorphism in Theorem 11.18.

More precise and down-to-earth proof of Theorem 11.18 is given as follows.

We recall that the map $\mathfrak{k}_{\mathfrak{b}}:\left(H\left(X ; \Lambda_{0}\right), \cup^{\mathfrak{b}}\right) \rightarrow \operatorname{Jac}\left(\mathfrak{P} \mathfrak{O}^{\mathfrak{b}}\right)$ is induced from the map

$$
\mathbf{p}_{i} \mapsto \sum_{k=0}^{\infty} \sum_{\ell_{1}=0}^{\infty} \sum_{\ell_{2}=0}^{\infty} \int_{L(\mathbf{u})} \mathfrak{q}_{\ell_{1}+\ell_{2}+1 ; k}\left(\mathfrak{b}^{\ell_{1}} \mathbf{p}_{i} \mathfrak{b}^{\ell_{2}}, b^{k}\right): \mathcal{A} \rightarrow \Lambda\left\langle\left\langle y, y^{-1}\right\rangle\right\rangle_{0}^{P} .
$$

(See (78).) Note $b=\sum x_{i} \mathbf{e}_{i}$ and the right hand side is a function of $x_{i}$. It then turns out to be a function of $y_{i}^{\mathbf{u}}=e^{x_{i}}$. Moreover by changing the variables to $y_{i}$ by the formula $y_{i}=T^{u_{i}} y_{i}^{\mathbf{u}}$, the right hand side becomes a function of $y_{i}$ and is an element of $\Lambda\left\langle\left\langle y, y^{-1}\right\rangle\right\rangle_{0}^{P}$.

We consider the case $\mathfrak{b}=\mathbf{0}$ for simplicity.

We consider the moduli space $\mathcal{M}_{k+1 ; 2}(\beta)$ of $J$-holomorphic disks with $k+1$ boundary and $\ell$ interior marked points, (See subsection 2.2.) and take a fiber product

$$
\mathcal{M}_{k+1 ; 2}^{\operatorname{main}}(\beta)_{\left(\mathrm{ev}_{1}^{+}, \mathrm{ev}_{2}^{+}\right)} \times\left(\mathbf{p} \times \mathbf{p}^{\prime}\right)
$$


where $\mathbf{p}, \mathbf{p}^{\prime} \in \mathcal{A}$. We denote this fiber product by

$$
\mathcal{M}_{k+1 ; 2}^{\operatorname{main}}\left(\beta ; \mathbf{p}, \mathbf{p}^{\prime}\right)
$$

Let $\mathcal{M}_{1 ; 2}$ be the moduli space of bordered Riemann surface of genus 0 with two interior and one boundary marked points. This moduli space is a two dimensional disk. We consider two points $\left[\Sigma_{1}\right],\left[\Sigma_{2}\right] \in \mathcal{M}_{1 ; 2}$ as in the figure below.

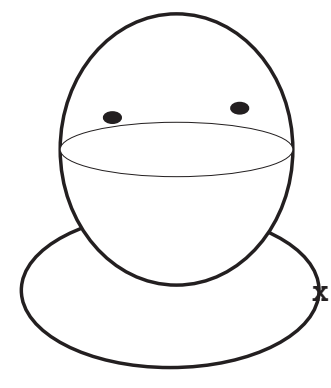

$\Sigma_{1}$

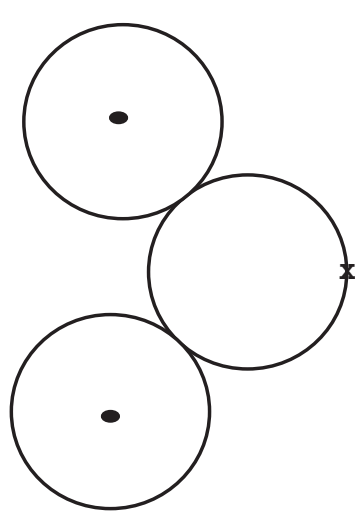

$\Sigma_{2}$

Figure 11.1

We have a forgetful map

$$
\text { forget }: \mathcal{M}_{k+1 ; 2}^{\operatorname{main}}(\beta) \rightarrow \mathcal{M}_{1 ; 2} .
$$

Namely we put

$$
\mathfrak{f o r g e t}\left(\left[\Sigma ; z_{0}, \ldots, z_{k}, z_{1}^{+}, z_{2}^{+}, u\right]\right)=\left[\Sigma ; z_{0} ; z_{1}^{+}, z_{2}^{+}\right] .
$$

It induces a map

$$
\text { forget : } \mathcal{M}_{k+1 ; 2}^{\text {main }}\left(\beta ; \mathbf{p}, \mathbf{p}^{\prime}\right) \rightarrow \mathcal{M}_{1 ; 2}
$$

For $i=1,2$, we denote by

$$
\mathcal{M}_{k+1 ; 2}^{\operatorname{main}}\left(\beta ; \mathbf{p}, \mathbf{p}^{\prime} ; \Sigma_{i}\right)
$$

the inverse image of $\left\{\left[\Sigma_{i}\right]\right\}$ in $\mathcal{M}_{k+1 ; 2}^{\text {main }}\left(\beta ; \mathbf{p}, \mathbf{p}^{\prime}\right)$.

Let $h_{j} \in H^{1}(L(\mathbf{u}) ; \mathbb{C})(j=1, \ldots, k)$. (Note we identify the cohomology group with the set of $T^{n}$ invariant forms.) We pull back $h_{1} \times \cdots \times h_{k}$ to $\mathcal{M}_{k+1 ; 2}^{\text {main }}\left(\beta ; \mathbf{p}, \mathbf{p}^{\prime} ; \Sigma_{i}\right)$ by $\left(\mathrm{ev}_{1}, \ldots, \mathrm{ev}_{k}\right)$ and consider the integration along fiber by $\mathrm{ev}_{0}$. We denote it by

$$
\operatorname{Corr}\left(h_{1} \times \cdots \times h_{k} ; \mathcal{M}_{k+1 ; 2}^{\text {main }}\left(\beta ; \mathbf{p}, \mathbf{p}^{\prime} ; \Sigma_{i}\right)\right) .
$$

More precisely we take a $T^{n}$ invariant multisection $\mathfrak{s}$ so that the zero set $\mathcal{M}_{k+1 ; 2}^{\text {main }}\left(\beta ; \mathbf{p}, \mathbf{p}^{\prime} ; \Sigma_{i}\right)^{\mathfrak{s}}$ is transversal to zero. Then integration along the fiber 
is well-defined. This is because $\mathrm{ev}_{0}$ on $\mathcal{M}_{k+1 ; 2}^{\text {main }}\left(\beta ; \mathbf{p}, \mathbf{p}^{\prime} ; \Sigma_{i}\right)^{\mathfrak{s}}$ must become a submersion by the $T^{n}$ equivariance.

We put

$$
\begin{aligned}
& \operatorname{Corr}\left(h_{1} \times \cdots \times h_{k} ; \mathcal{M}_{k+1 ; 2}^{\text {main }}\left(\mathbf{p}, \mathbf{p}^{\prime} ; \Sigma_{i}\right)\right) \\
& \quad=\sum_{\beta} T^{(\beta \cap \omega) / 2 \pi} \operatorname{Corr}\left(h_{1} \times \cdots \times h_{k} ; \mathcal{M}_{k+1 ; 2}^{\text {main }}\left(\beta ; \mathbf{p}, \mathbf{p}^{\prime} ; \Sigma_{i}\right)\right)
\end{aligned}
$$

and extend $\operatorname{Corr}\left(\cdots ; \mathcal{M}_{k+1 ; 2}^{\text {main }}\left(\mathbf{p}, \mathbf{p}^{\prime} ; \Sigma_{i}\right)\right)$ to

$$
H^{1}\left(L(\mathbf{u}) ; \Lambda_{0}\right)^{\otimes k} \rightarrow \Lambda_{0}
$$

We then can prove the following two formulas:

$$
\begin{aligned}
& \operatorname{Corr}(\underbrace{b, \ldots, b}_{k} ; \mathcal{M}_{k+1 ; 2}^{\text {main }}\left(\mathbf{p}, \mathbf{p}^{\prime} ; \Sigma_{1}\right))=\mathfrak{q}_{1 ; k}\left(\mathbf{p} \cup^{Q} \mathbf{p}^{\prime} ; b^{k}\right) . \\
& \operatorname{Corr}(\underbrace{b, \ldots, b}_{k} ; \mathcal{M}_{k+1 ; 2}^{\text {main }}\left(\mathbf{p}, \mathbf{p}^{\prime} ; \Sigma_{2}\right))=\sum_{k_{1}+k_{2}=k} \mathfrak{q}_{1 ; k_{1}}\left(\mathbf{p} ; b^{k_{1}}\right) \mathfrak{q}_{1 ; k_{2}}\left(\mathbf{p}^{\prime} ; b^{k_{2}}\right) .
\end{aligned}
$$

Note the sum over $k$ of the right hand sides of (85) and (86) are

$$
\mathfrak{k}_{\mathfrak{s}_{0}}\left(\mathbf{p} \cup \cup^{Q} \mathbf{p}^{\prime}\right) \quad \text { and } \quad \mathfrak{k}_{\mathfrak{s}_{0}}(\mathbf{p}) \mathfrak{k}_{\mathfrak{s}_{0}}\left(\mathbf{p}^{\prime}\right)
$$

respectively. (Note we are studying the case $\mathfrak{b}=\mathbf{0}$.)

We finally use cobordism argument to show that the left hand side of (85) coincides with the left hand side of (86) modulo elements in the Jacobian ideal. This is an outline of the proof of Theorem 11.18. See [FOOO5] section 2.6 for detail.

The outline of the rest of the proof of Theorem 11.6 is as follows.

We first prove the surjectivity of $\mathfrak{k}_{\mathfrak{b}}$. For this purpose we consider the map obtained by reducing the coefficient to $\mathbb{C}=\Lambda_{0} / \Lambda_{+}$. Then the quantum cohomology of the domain becomes ordinary cohomology. We can calculate the $\mathbb{C}=\Lambda_{0} / \Lambda_{+}$reduction of the Jacobian ring using Cho-Oh's result (namely by studying the leading order term $z_{1}+\cdots+z_{m}$. See Theorem 8.2.) Then the $\mathbb{C}$-reduction of $\mathfrak{k}_{\mathfrak{b}}$ is an isomorphism by a classical result of Stanley which calculates the cohomology ring of toric manifold. (See for example [Ful].) It implies that $\mathfrak{k}_{\mathfrak{b}}$ itself is surjective.

We remark that the fact that $\mathbb{C}$-reduction of $\mathfrak{k}_{\mathfrak{s}_{\mathfrak{b}}}$ is an isomorphism does not imply that $\mathfrak{k}_{\mathfrak{G}_{\mathfrak{b}}}$ is isomorphism. In fact we need to eliminate the possibility that $\operatorname{Jac}\left(\mathfrak{P O}^{\mathfrak{b}}\right)$ has a component such as $\Lambda_{0} /\left(T^{\lambda}\right)$. Note that the (quantum) cohomology $H\left(X ; \Lambda_{0}\right)$ is a free $\Lambda_{0}$ module. Therefore to prove the injectivity of $\mathfrak{k}_{\mathfrak{b}}$ and complete the proof of Theorem 11.6 it suffices to prove the following inequality.

$$
\operatorname{rank}_{\Lambda}\left(\operatorname{Jac}\left(\mathfrak{P O}^{\mathfrak{b}}\right) \otimes_{\Lambda_{0}} \Lambda\right) \geq \operatorname{rank}_{\mathbb{Q}} H(X ; \mathbb{Q}) .
$$

We remark that in many explicit examples we can prove the equality (87) directly by finding critical points of $\mathfrak{P O}^{\mathfrak{b}}$, for example by solving leading 
term equation. However the proof of $(87)$ is in general more involved, which we briefly describe now. We consider the case $\mathfrak{b}=\mathbf{0}$, for simplicity.

We prove (87) in two steps. We first use a result of McDuff-Tolman [MT] (which is based on Seidel's work [Se1]), to find elements $z_{1}^{\prime}, \ldots, z_{m}^{\prime} \in$ $Q H\left(X ; \Lambda_{0}\right)$ with the following properties.

1) $z_{1}^{\prime}, \ldots, z_{m}^{\prime}$ satisfies quantum Stanley-Reisner relation.

2) There exists $\mathfrak{P}_{i}\left(Z_{1}, \ldots, Z_{m}\right)=\sum_{j=1}^{m} v_{j, i} Z_{i}+\sum_{k=1}^{\infty} T^{\rho_{k}} P_{i, k}\left(Z_{1}, \ldots\right.$, $\left.Z_{m}\right)$ such that

$$
\mathfrak{P}_{i}\left(z_{1}^{\prime}, \ldots, z_{m}^{\prime}\right)=0
$$

and $\rho_{k} \rightarrow \infty, \rho_{k}>0, P_{i, k} \in \mathbb{C}\left[Z_{1}, \ldots, Z_{m}\right]$. (We recall $d \ell_{j}=\left(v_{j, 1}, \ldots\right.$, $\left.v_{j, n}\right) \in \mathbb{Z}^{n}$.)

3) The relations in the above $(1),(2)$ are all the relations among $z_{i}^{\prime}$. Moreover $z_{i}^{\prime}$ generates $Q H\left(X ; \Lambda_{0}\right)$.

Let us explain the above statement briefly. By putting $Z_{i}=T^{\lambda_{i}} y_{1}^{v_{i, 1}} \cdots y_{n}^{v_{i, n}}$ we obtain a surjective ring homomorphism

$$
\Lambda\left[Z_{1}, \ldots, Z_{m}\right] \rightarrow \Lambda\left[y_{1}, y_{1}^{-1}, \ldots, y_{n}, y_{n}^{-1}\right] .
$$

The quantum Stanley-Reisner relations are the generators of the kernel of this homomorphism. (See [FOOO3] Definition 6.4.) The quantum StanleyReisner relation appeared in the Batyrev's work on quantum cohomology of toric manifold and is given explicitly by using moment polytope $P$.

We put $z_{i}=T^{\lambda_{i}} y_{1}^{v_{i, 1}} \cdots y_{n}^{v_{i, n}} \in \operatorname{Jac}\left(\mathfrak{P O}^{\mathbf{0}}\right)$. Then (47) implies that it satisfies the formula

$$
\sum_{j=1}^{m} v_{j, i} z_{i}+\sum_{k=1}^{\infty} T^{\rho_{k}} \frac{\partial P_{k}}{\partial x_{i}}\left(z_{1}, \ldots, z_{m}\right)=0
$$

(Note we put $y_{i}=e^{x_{i}}$ so $(89)$ is $\frac{\partial P_{k}}{\partial x_{i}}=y_{i} \frac{\partial P_{k}}{\partial y_{i}}$.) We remark that the first term of the left hand side of (89) is

$$
\frac{\partial \mathfrak{P \mathfrak { D } ^ { 0 }}}{\partial x_{i}}\left(z_{1}, \ldots, z_{m}\right)
$$

We also remark that the left hand side of (88) is similar to (89). Namely their leading order terms coincide.

The element $z_{i}^{\prime}$ is the invariant of [Se1] associated to the Hamiltonian $S^{1}$ action. Here $S^{1}$ is the component of $T^{n}$ which fixes $D_{i}$. The fact that they satisfy the quantum Stanley-Reisner relation is proved in $[\mathbf{M T}]$ using the relation between those $S^{1}$ actions and basic properties of Seidel invariant. The property $(2)$ can be proved using the fact $z_{i}^{\prime} \equiv\left[D_{i}\right] \bmod \Lambda_{+}$. 
Let $(Q S R) \subset \Lambda_{0}\left\langle\left\langle Z_{1}, \ldots, Z_{m}\right\rangle\right\rangle$ be the ideal generated by the quantum Stanley-Reisner relations. Then (1), (2) above imply the existence of homomorphism

$$
\frac{\Lambda_{0}\left\langle\left\langle Z_{1}, \ldots, Z_{m}\right\rangle\right\rangle}{\operatorname{Clos}\left((Q S R) \cup\left\{\mathfrak{P}_{i}: i=1, \ldots, m\right\}\right)} \rightarrow Q H\left(X ; \Lambda_{0}\right)
$$

Here Clos means a closure with respect to an appropriate topology. By reduction to $\mathbb{C}=\Lambda_{0} / \Lambda_{+}$we can show that (90) is an isomorphism. (We use the fact that $Q H\left(X ; \Lambda_{0}\right)$ is torsion free here.)

Now the proof of $(87)$ goes as follows. For $\mathfrak{s} \in \Lambda$ we put

$$
\mathfrak{P}_{i}^{\mathfrak{s}}=\mathfrak{s} \frac{\partial \mathfrak{P O ^ { 0 }}}{\partial x_{i}}+(1-\mathfrak{s}) \mathfrak{P}_{i}
$$

We remark $\mathfrak{P}_{i}^{\mathfrak{s}}$ has the form

$$
\mathfrak{P}_{i}^{\mathfrak{s}}\left(Z_{1}, \ldots, Z_{m}\right)=\sum_{j=1}^{m} v_{j, i} Z_{i}+\sum_{k=1}^{\infty} T^{\rho_{k}} P_{i, k}^{\mathfrak{s}}\left(Z_{1}, \ldots, Z_{m}\right)
$$

We define the ring $\mathfrak{R}_{\mathfrak{s}}$ by

$$
\mathfrak{R}_{\mathfrak{s}}=\frac{\Lambda_{0}\left\langle\left\langle Z_{1}, \ldots, Z_{m}\right\rangle\right\rangle}{\operatorname{Clos}\left((Q S R) \cup\left\{\mathfrak{P}_{i}^{\mathfrak{s}}: i=1, \ldots, m\right\}\right)} \otimes_{\Lambda_{0}} \Lambda .
$$

We have

$$
\mathfrak{R}_{0} \cong Q H(X ; \Lambda)
$$

since (90) is an isomorphism. On the other hand

$$
\mathfrak{R}_{1} \cong \operatorname{Jac}\left(\mathfrak{P O}^{\mathbf{0}}\right) \otimes_{\Lambda_{0}} \Lambda .
$$

Thus it suffices to show that $\operatorname{dim}_{\Lambda} \mathfrak{R}_{\mathfrak{s}}$ is independent of $\mathfrak{s}$. We regard $\cup_{\mathfrak{s} \in \Lambda}$ $\operatorname{Spec}\left(\Re_{\mathfrak{s}}\right)$ as a family of affine schemes parametrized by $\mathfrak{s} \in \Lambda$. If we can prove that this family is flat and proper then the independence of $\operatorname{dim}_{\Lambda} \mathfrak{R}_{\mathfrak{s}}$ is a standard result of algebraic geometry.

We prove the properness using the fact that the valuation of the solution of the equation $\mathfrak{P}_{1}^{\mathfrak{s}}=\cdots=\mathfrak{P}_{m}^{\mathfrak{s}}=0$ can not escape from moment polytope. The flatness is a consequence of the fact that our scheme is a local complete intersection and also of standard facts about the regular sequence of CohenMacauley ring.

In general $\mathfrak{P}_{i}^{\mathfrak{s}}$ is an infinite series rather than a polynomial. So we first need to change the coordinate $y_{i}$ so that $\mathfrak{P}_{i}^{\mathfrak{s}}$ becomes a polynomial. Such a process is known in algebraic geometry as a algebraization of singularity. See [FOOO5] section 2.9.

This is an outline of the proof of (87). See [FOOO5] especially its section 2.11 for details. 
REMARK 11.19. We regard

$$
\bigcup_{\mathfrak{b} \in H\left(X ; \Lambda_{0}\right)} \operatorname{Spec}\left(\mathfrak{P O}^{\mathfrak{b}}\right)
$$

as a $H\left(X ; \Lambda_{0}\right)$ parametrized 'family of schemes'4

The same argument to show the flatness and properness of the family $\cup_{\mathfrak{s} \in \Lambda} \operatorname{Spec}\left(\mathfrak{R}_{\mathfrak{s}}\right)$ seems to be applicable to show that the family (91) is also flat and proper.

In the study of K. Saito theory of Laurent polynomials (such as one described in $[\mathbf{S a b}])$, the properness of the family of the critical point sets is an important issue. When one works over $\mathbb{C}$ the properness is not necessarily satisfied. When we work over a Novikov ring in place of $\mathbb{C}$, properness of the family of the critical point sets (that is the geometric points of $\operatorname{Spec}\left(\mathfrak{P O}^{\mathfrak{b}}\right)$ ) is always satisfied at least for the potential function appearing as the mirror of a toric manifold. The authors believe that this is an important advantage of working with Novikov ring over working with $\mathbb{C}$.

REMARK 11.20. Let us consider the family (91). For the $H^{2}\left(X ; \Lambda_{0}\right)$ part of $\mathfrak{b}$ it is natural to replace the coordinate $w_{i}$ by its exponential $\mathfrak{w}_{i}=e^{w_{i}}$. Then we may extend the domain $\left\{\mathfrak{w}_{i} \mid \mathfrak{v}_{T}\left(\mathfrak{w}_{i}\right)=0\right\}$ to $\mathfrak{w}_{i} \in \Lambda$. Note in $\mathfrak{P O}^{\mathfrak{b}}$ the leading order term is $\sum \mathfrak{w}_{i} z_{i}$. So if we extend $\mathfrak{w}_{i}$ and allow for example $\mathfrak{w}_{i}=T^{c}$, we have a term $T^{c} z_{i}$. We may regard this insertion $\mathfrak{w}_{i}=T^{c}$ as changing the moment polytope. Namely appearance of the term $T^{c} z_{i}$ is equivalent to moving $\partial_{i} P=\left\{\mathbf{u} \mid \ell_{i}(\mathbf{u})=0\right\}$ to $\left\{\mathbf{u} \mid \ell_{i}(\mathbf{u})=-c\right\}^{5}$.

Thus for this extended family the flatness and properness still hold as far as the corresponding moment polytope is combinatorially equivalent to the original one.

There is some flavor of this kind of arguments in [FOOO5] subsection 2.11.2.

\section{Poincaré duality and Residue pairing}

In this section we explain that the isomorphism in Theorem 11.6 can be enhanced to give an isomorphism between two Frobenius manifold structures.

\subsection{Big quantum cohomology and Frobenius manifold.}

Definition 12.1. A Frobenius manifold structure on a manifold $M$ is a quintet $(\langle\cdot\rangle, \nabla, \circ, e, \Phi)$ with the following properties.

\footnotetext{
${ }^{4}$ It is proved in [FOOO5] that each of $\mathfrak{P O}^{\mathfrak{b}}$ can be transformed to a Laurent polynomial by change of variables. So we can define its Spec. It is not verified that the whole family can be regarded to be a scheme. So we put quotation mark.

${ }^{5}$ In other words the parameter $\mathfrak{v}_{T}\left(\mathfrak{w}_{i}\right)$ corresponds to the Kähler cone of our toric manifold $X$. This is similar to the fact that the valuation of $\mathfrak{y}_{i}$ corresponds to the parameter $\mathbf{u}$ of the Lagrangian submanifold $L(\mathbf{u})$
} 
1) $\langle\cdot\rangle$ is a non-degenerate inner product on the tangent bundle $T M$.

2) $\nabla$ is a connection of $T M$.

3) $\nabla$ is a metric connection. Namely :

$$
X\langle Y, Z\rangle=\left\langle\nabla_{X} Y, Z\right\rangle+\left\langle Y, \nabla_{X} Z\right\rangle .
$$

4) $\nabla$ is flat and torsion free. Namely :

$$
\begin{array}{r}
\nabla_{X} \nabla_{Y}-\nabla_{Y} \nabla_{X}-\nabla_{[X, Y]}=0 \\
\nabla_{X} Y-\nabla_{Y} X-[X, Y]=0 .
\end{array}
$$

5) $\circ$ defines a ring structure on $T_{p} M$ which depends smoothly on $p$ and satisfies

$$
\langle X \circ Y, Z\rangle=\langle X, Y \circ Z\rangle .
$$

An associative algebra with unit which satisfies (92) is called a Frobenius algebra.

$6) e$ is a section of $T M$ such that $e(p)$ is the unit of $\left(T_{p} M, \circ,+\right)$ for each $p$. Moreover

$$
\nabla e=0 .
$$

7) $\Phi$ is a function on $M$ such that

$$
\left\langle\frac{\partial}{\partial x_{i}} \circ \frac{\partial}{\partial x_{j}}, \frac{\partial}{\partial x_{k}}\right\rangle=\frac{\partial^{3} \Phi}{\partial x_{i} \partial x_{j} \partial x_{k}} .
$$

Here $x_{i}(i=1, \ldots, \operatorname{dim} M)$ is a local coordinate of $M$ such that $\nabla_{\frac{\partial}{\partial x_{i}}}\left(\frac{\partial}{\partial x_{j}}\right)=0$. We call $\Phi$ the potential.

In some case we have a vector field $\mathfrak{E}$ on $M$ that satisfies the following

$$
\begin{aligned}
\mathfrak{E}\langle X, Y\rangle-\langle[\mathfrak{E}, X], Y\rangle-\langle X,[\mathfrak{E}, Y]\rangle & =d_{1}\langle X, Y\rangle, \\
{[\mathfrak{E}, X \circ Y]-[\mathfrak{E}, X] \circ Y-X \circ[\mathfrak{E}, Y] } & =d_{2} X \circ Y, \\
{[\mathfrak{E}, e] } & =d_{3} e,
\end{aligned}
$$

where $d_{1}, d_{2}, d_{3} \in \mathbb{Q}$. We call $\mathfrak{E}$ the Euler vector field.

REMARK 12.2. In various situations where a Frobenius manifold arises the tangent space $T_{p} M$ appears as either a $\mathbb{C}$ vector space or a $\Lambda$ vector space. In that case the inner product $\langle\cdot\rangle$ is bilinear over $\mathbb{C}$ or $\Lambda$. (In this case $\langle\cdot\rangle$ is required to be complex symmetric not hermitian.) Moreover $\Phi$ is a $\mathbb{C}$ or $\Lambda$ valued function.

We do not try to define what connection, function, coordinate etc. mean in case $T M$ is a $\Lambda$ vector space. At the present stage of development, we do not meet the situation where we need to seriously study it. In the main example of our consideration, $M$ is a $\Lambda_{0}$ affine space, hence we can easily make sense out of them. 
This structure first appeared in K. Saito's work [Sa] (see the next subsection). Dubrovin [Dub] discovered this structure in Gromov-Witten theory, which we recall below.

Let $X$ be a symplectic manifold. We take $M=H^{\text {evev }}\left(X ; \Lambda_{0}\right)$ the even degree cohomology group of $X$ with $\Lambda_{0}$ coefficients. (We may include odd degree part by regarding $X$ as a supermanifold. Since in the case of our main interest (toric manifold), there is no cohomology class of odd degree, we do not discuss odd degree part.)

In subsection 11.2 we associate a deformed quantum cup product $\cup^{\mathfrak{b}}$ on $H(X ; \Lambda)$ for each $\mathfrak{b} \in H^{\text {even }}\left(X ; \Lambda_{0}\right)$. We regard $T_{\mathfrak{b}} M=H(X ; \Lambda)$ and put $\circ=\cup^{\mathfrak{b}}$ there. It is associative.

REMARK 12.3. Note $H^{\text {even }}\left(X ; \Lambda_{0}\right)$ is not an open set of $H^{\text {even }}(X ; \Lambda)$. So $T_{\mathfrak{b}} H^{\text {even }}\left(X ; \Lambda_{0}\right)=H(X ; \Lambda)$ does not make sense in a usual sense of manifold. This is regarded only as a convention here.

We have Poincaré duality pairing

$$
H^{d}(X ; \Lambda) \otimes_{\Lambda} H^{2 n-d}(X ; \Lambda) \rightarrow \Lambda .
$$

The inner product $\langle\cdot\rangle$ is the Poincaré duality pairing. We remark that then the Levi-Civita connection, that is the connection which is a torsion free metric connection of the metric $\langle\cdot\rangle$, is the standard affine connection of the vector space $H^{\text {even }}\left(X ; \Lambda_{0}\right)$. It is obviously flat.

(92) follows from

$$
\left\langle\mathfrak{c} \cup^{\mathfrak{b}} \mathfrak{d}, \mathfrak{e}\right\rangle_{\operatorname{PD}_{X}}=\sum_{\ell=0}^{\infty} \frac{1}{\ell !} G W_{\ell+3}(\mathfrak{c}, \mathfrak{d}, \mathfrak{e}, \mathfrak{b}, \ldots, \mathfrak{b}) .
$$

(See (69).) and the fact that $G W_{\ell}\left(Q_{1}, \ldots, Q_{\ell}\right)$ is independent of the permutation of $Q_{i}$.

The element $e$ is the unit of the cohomology group that is the Poincaré dual to the fundamental homology class $[X]$.

The potential $\Phi$ is defined by

$$
\Phi(\mathfrak{b})=\sum_{\ell=0}^{\infty} \frac{1}{\ell !} G W_{\ell}(\mathfrak{b}, \ldots, \mathfrak{b})
$$

for which the formula (93) can be easily checked. The potential $\Phi$ in (95) is called the Gromov-Witten potential.

The Euler vector field $\mathfrak{E}$ is defined by the vector field:

$$
\mathfrak{E}=\frac{\partial}{\partial w_{0}}+\sum_{i=1}^{m} r_{i} \frac{\partial}{\partial \mathfrak{w}_{i}}+\sum_{i=m+1}^{B}\left(1-\frac{\operatorname{deg} \mathbf{p}_{i}}{2}\right) w_{i} \frac{\partial}{\partial w_{i}},
$$

where $c_{1}(X)=\sum_{i=1}^{m} r_{i} \mathbf{p}_{i}$. We remark that $\mathbf{p}_{i}, i=0, \ldots, B$ are basis of $H^{\text {even }}$ $(X ; \mathbb{Q})$ such that $\operatorname{deg} \mathbf{p}_{0}=0, \operatorname{deg} \mathbf{p}_{i}=2$ for $i=1, \ldots, m$ and $\operatorname{deg} \mathbf{p}_{i}>2$ for $i>m$. 
By using the dimension formula

$$
\operatorname{dim}_{\mathbb{C}} \mathcal{M}_{\ell}(\alpha)=n+\ell-3+c_{1}(X) \cap \alpha
$$

of the moduli space $\mathcal{M}_{\ell}(\alpha)$ of pseudo-holomorphic spheres with $\ell$ interior marked points and of homology class $\alpha$, we can prove (94), where $d_{1}=2-n$, $d_{2}=1, d_{3}=0$. Thus we have:

TheOrem 12.4. (Dubrovin) $\left(\langle\cdot\rangle, \nabla, \cup^{\mathfrak{b}}, \Phi, e\right)$ is a structure of Frobenius manifold on $H\left(X ; \Lambda_{0}\right)$. (96) is its Euler vector field.

\subsection{A fragment of K. Saito theory. Let}

$$
F\left(x_{1}, \ldots, x_{n} ; w_{0}, w_{1}, \ldots, w_{B}\right): U \times V \rightarrow \mathbb{C}
$$

be a holomorphic function on $U \times V \subset \mathbb{C}^{n} \times \mathbb{C}^{B+1}$. Here $U$ and $V$ are small neighborhoods of origin in $\mathbb{C}^{n}$ and $\mathbb{C}^{B+1}$, respectively.

We assume $F$ is of the form

$$
F\left(x_{1}, \ldots, x_{n} ; w_{0}, w_{1}, \ldots, w_{B}\right)=w_{0}+F\left(x_{1}, \ldots, x_{n} ; 0, w_{1}, \ldots, w_{B}\right) .
$$

We put

$$
F^{\vec{w}}\left(x_{1}, \ldots, x_{n}\right)=F\left(x_{1}, \ldots, x_{n} ; w_{0}, w_{1}, \ldots, w_{B}\right),
$$

for $\vec{w}=\left(w_{0}, \ldots, w_{B}\right)$. We assume that $F^{\overrightarrow{0}}\left(x_{1}, \ldots, x_{n}\right)$ has $\vec{x}=\overrightarrow{0}$ as an isolated critical point. Namely $\left(d F^{\overrightarrow{0}}\right)(0, \ldots, 0)=0$, and $\left(d F^{\overrightarrow{0}}\right)(\vec{x}) \neq 0$ for $\vec{x} \in$ $U \backslash\{\overrightarrow{0}\}$.

Definition 12.5. We define the Jacobian $\operatorname{ring} \operatorname{Jac}\left(F^{\vec{w}}\right)$ by

$$
\operatorname{Jac}\left(F^{\vec{w}}\right)=\frac{\mathcal{O}(U)}{\left(\frac{\partial F^{\vec{w}}}{\partial x_{i}} ; i=1, \ldots, n\right)} .
$$

Here $\mathcal{O}(U)$ is the ring of holomorphic functions on $U$ and the denominator is its ideal generated by $\frac{\partial F^{\vec{w}}}{\partial x_{i}}, i=1, \ldots, n$.

We define the Kodaira-Spencer map $\mathfrak{k}_{\vec{w}}: T_{\vec{w}} V \rightarrow \operatorname{Jac}\left(F^{\vec{w}}\right)$ by

$$
\mathfrak{k}_{\vec{w}}\left(\frac{\partial}{\partial w_{i}}\right) \equiv \frac{\partial F}{\partial w_{i}}\left(x_{1}, \ldots, x_{n} ; \vec{w}\right) \in \operatorname{Jac}\left(F^{\vec{w}}\right) .
$$

$F$ is called a universal unfolding of $F^{\overrightarrow{0}}$ if $\mathfrak{k}_{\overrightarrow{0}}: T_{\overrightarrow{0}} V \rightarrow \operatorname{Jac}\left(F^{\overrightarrow{0}}\right)$ is an isomorphism.

We remark that if $F$ is a universal unfolding of $F^{\overrightarrow{0}}$ then by shrinking $V$ if necessary we may assume that $\mathfrak{k}_{\mathfrak{w}}$ is an isomorphism for any $\vec{w} \in V$. We assume it in the rest of this subsection.

We remark that $\operatorname{Jac}\left(F^{\vec{w}}\right)$ is a ring. On the other hand $T_{\vec{w}} V$ does not have a ring structure a priori. We define

$$
X \circ Y=\left(\mathfrak{k}_{\vec{w}}\right)^{-1}\left(\mathfrak{k}_{\mathfrak{s}_{\vec{w}}}(X) \mathfrak{k}_{\vec{w}}(Y)\right),
$$


for $X, Y \in T_{\vec{w}} V$. Thus $\left(T_{\vec{w}} V, \circ,+\right)$ forms a ring. Note $\partial / \partial w_{0} \in T_{\vec{w}} V$ is sent to $[1] \in \operatorname{Jac}\left(F^{\vec{w}}\right)$. Therefore

$$
e(\vec{w})=\partial / \partial w_{0} \in T_{\vec{w}} V
$$

is a unit.

Theorem 12.6. (K.Saito-M.Saito) There exists a $\mathbb{C}$ valued metric $\langle\cdot\rangle$ on $T V$, its Levi-Civita connection $\nabla$ and a holomorphic function $\Phi: V \rightarrow \mathbb{C}$ such that $(\langle\cdot\rangle, \circ, e, \nabla, \Phi)$ is a Frobenius manifold.

K. Saito [Sa] constructed a Frobenius manifold structure assuming the existence of a primitive form. We do not explain the notion of primitive form here. (See $[\mathbf{S a T a}]$ for its description in a way closely related to the discussion here.) Existence of primitive form for a universal unfolding of a germ of isolated singularity is established in [MSa]. We remark that Theorem 12.6 had been proved before Gromov-Witten theory started.

The metric $\langle\cdot\rangle$ is called a residue paring. Since $\nabla$ is flat there exists a local coordinate $t_{0}, t_{1}, \ldots, t_{B}$ of $V$ so that $\nabla_{\partial / \partial t_{i}}\left(\partial / \partial t_{j}\right)=0$. Such a coordinate $\left(t_{0}, t_{1}, \ldots, t_{B}\right)$ is called a flat coordinate. $\left(t_{0}=w_{0}.\right)$

For some $F$ associated to an ADE singularity, the primitive form takes a simple form $d x_{1} \wedge d x_{2} \wedge d x_{3}$. In such a case we have the following description of the residue pairing.

We put

$$
\operatorname{Crit}\left(F^{\vec{w}}\right)=\left\{\mathfrak{y} \in U \mid d F^{\vec{w}}(\mathfrak{y})=0\right\} .
$$

Let $\mathcal{O}_{\mathfrak{y}}$ be the ring of germs of holomorphic functions at $\mathfrak{y} \in U$. We put

$$
\operatorname{Jac}\left(F^{\vec{w}} ; \mathfrak{y}\right)=\frac{\mathcal{O}_{\mathfrak{y}}}{\left(\frac{\partial F^{\vec{w}}}{\partial x_{i}} ; i=1, \ldots, n\right)} .
$$

The following fact is standard:

Proposition 12.7. We have

$$
\operatorname{Jac}\left(F^{\vec{w}}\right) \cong \prod_{\mathfrak{y} \in \operatorname{Crit}\left(F^{\vec{w}}\right)} \operatorname{Jac}\left(F^{\vec{w}} ; \mathfrak{y}\right) .
$$

$\operatorname{Jac}\left(F^{\vec{w}} ; \mathfrak{y}\right)$ is one dimensional if and only if the critical point $\mathfrak{y}$ is nondegenerate.

Let $\vec{w}$ be a vector such that $F^{\vec{w}}$ is a Morse function. Let $1_{\mathfrak{y}} \in \operatorname{Jac}\left(F^{\vec{w}} ; \mathfrak{y}\right)$ be the unit. Then Proposition 12.7 implies that $\left\{1_{\mathfrak{y}} \mid \mathfrak{y} \in \operatorname{Crit}\left(F^{\vec{w}}\right)\right\}$ forms a $\mathbb{C}$ basis of the vector $\operatorname{space} \operatorname{Jac}\left(F^{\vec{w}}\right)$. If $\mathfrak{y} \neq \mathfrak{y}^{\prime}$ we obtain

$$
\left\langle 1_{\mathfrak{y}}, 1_{\mathfrak{y}^{\prime}}\right\rangle=\left\langle 1_{\mathfrak{y}}, 1_{\mathfrak{y}^{\prime}} \circ 1\right\rangle=\left\langle 1_{\mathfrak{y}} \circ 1_{\mathfrak{y}^{\prime}}, 1\right\rangle=0,
$$

from the equation $1_{\mathfrak{y}} \circ 1_{\mathfrak{y}^{\prime}}=0$ and (92). Namely $\left\{1_{\mathfrak{y}} \mid \mathfrak{y} \in \operatorname{Crit}\left(F^{\vec{w}}\right)\right\}$ is an orthogonal basis with respect to the residue pairing. 
LEMMA 12.8. If the primitive form is $d x_{1} \wedge \cdots \wedge d x_{n}$ and $F^{\vec{w}}$ is a Morse function then we have

$$
\left\langle 1_{\mathfrak{y}}, 1_{\mathfrak{y}}\right\rangle=\left(\operatorname{det}\left[\frac{\partial^{2} F^{\vec{w}}}{\partial x_{i} \partial x_{j}}\right]_{i=1, j=1}^{i=n, j=n}(\mathfrak{y})\right)^{-1} .
$$

This lemma follows from the definition. We remark that in general the primitive form is not necessarily equal to $d x_{1} \wedge \cdots \wedge d x_{n}$.

12.3. Residue pairing on $\operatorname{Jac}\left(\mathfrak{P O}^{\mathfrak{b}}\right)$. We now consider the case $F\left(x_{1}, \ldots, x_{n}, \vec{w}\right)=\mathfrak{P O}^{\mathfrak{b}}\left(y_{1}, \ldots, y_{n}\right)$ where $\mathfrak{b}=\sum w_{i} \mathbf{p}_{i}$ and $e^{x_{i}}=y_{i}$.

We however remark that our situation is different from that of subsection 12.2 in the following two points.

1) The tangent space $T_{\mathfrak{b}}\left(H\left(X ; \Lambda_{0}\right)\right)$ is a $\Lambda$ vector space and is not a $\mathbb{C}$ vector space.

2) The 'open set' on which $\mathfrak{P O}^{\mathfrak{b}}$ is defined is the set $\mathfrak{A}(\stackrel{\circ}{P})$ which is not a 'small' neighborhood of a point.

However, many parts of the story are directly translated to the case $\mathfrak{P O}^{\mathfrak{b}}$. (See however Remark 12.26.) Note $V$ in subsection 12.2 corresponds to $H\left(X ; \Lambda_{0}\right)$.

In this subsection we describe a pairing on $\operatorname{Jac}\left(\mathfrak{P O}^{\mathfrak{b}}\right)$ which we expect to be the version of residue pairing in our situation.

Definition 12.9. Let $C$ be a $\mathbb{Z}_{2}$ graded finitely generated free $\Lambda$ module. A structure of unital Frobenius algebra of dimension $n$ is $\langle\cdot, \cdot\rangle: C^{k} \otimes C^{n-k} \rightarrow$ $\Lambda, \cup: C^{k} \otimes C^{\ell} \rightarrow C^{k+\ell}, 1 \in C^{0}$, such that:

1) $\langle\cdot, \cdot\rangle$ is a graded symmetric bilinear form which induces an isomorphism $x \mapsto(y \mapsto\langle x, y\rangle), C^{k} \rightarrow \operatorname{Hom}_{\Lambda}\left(C^{n-k}, \Lambda\right)$.

2) $\cup$ is an associative product on $C$. 1 is its unit.

3) $\langle x \cup y, z\rangle=\langle x, y \cup z\rangle$.

The cohomology group of an oriented closed manifold becomes a unital Frobenius algebra in an obvious way.

Definition 12.10. Let $(C,\langle\cdot, \cdot\rangle, \cup, 1)$ be a unital Frobenius algebra. We take a basis $\mathbf{e}_{I}, I \in \mathfrak{I}$ of $C$ such that $\mathbf{e}_{0}$ is the unit. Let $g_{I J}=\left\langle\mathbf{e}_{I}, \mathbf{e}_{J}\right\rangle$ and let $g^{I J}$ be its inverse matrix. We define an invariant of $C$ by

$$
\begin{aligned}
Z(C)= & \sum_{\substack{I_{1}, I_{2}, I_{3} \in \mathfrak{I}\\
}} \sum_{J_{1}, J_{2}, J_{3} \in \mathfrak{I}}(-1)^{*} g^{I_{1} J_{1}} g^{I_{2} J_{2}} g^{I_{3} 0} g^{J_{3} 0} \\
& \times\left\langle\mathbf{e}_{I_{1}} \cup \mathbf{e}_{I_{2}}, \mathbf{e}_{I_{3}}\right\rangle\left\langle\mathbf{e}_{J_{1}} \cup \mathbf{e}_{J_{2}}, \mathbf{e}_{J_{3}}\right\rangle
\end{aligned}
$$

where $*=\operatorname{deg} \mathbf{e}_{I_{1}} \operatorname{deg} \mathbf{e}_{J_{2}}+\frac{n(n-1)}{2}$. We call $Z(C)$ the trace of unital Frobenius algebra $C$. 
It is straightforward to check that $Z(C)$ is independent of the choice of the basis. This invariant is an example of 1-loop partition function and can be described by the following Feynman diagram.

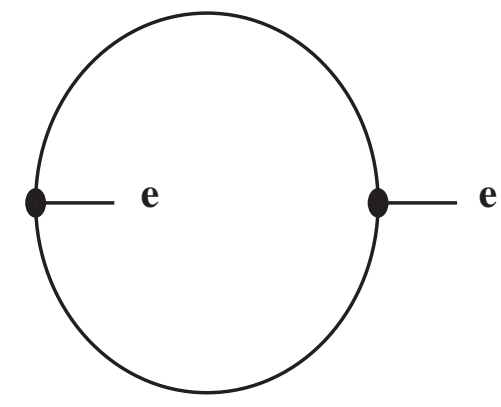

FiguRE 12.1

Let us consier $\mathbf{u} \in \operatorname{Int} P$ and $b \in H^{1}\left(L(\mathbf{u}) ; \Lambda_{0}\right)$ such that the Floer cohomology $H F((L(\mathbf{u}),(\mathfrak{b}, b)),(L(\mathbf{u}),(\mathfrak{b}, b)) ; \Lambda)$ is isomorphic to $H\left(T^{n} ; \Lambda\right)$.

We have a binary operator $\mathfrak{m}_{2}^{\mathfrak{c}, \mathfrak{b}, b}$ on it. The Poincaré duality induces a $\Lambda$ valued non-degenerate inner product $\langle\cdot\rangle_{\mathrm{PD}_{L(\mathbf{u})}}$ of it.

We define

$$
\begin{aligned}
x \cup^{\mathfrak{c}, \mathfrak{b}, b} y & =(-1)^{\operatorname{deg} x(\operatorname{deg} y+1)} \mathfrak{m}_{2}^{\mathfrak{c}, \mathfrak{b}, b}(x, y), \\
\langle x, y\rangle_{\text {cyc }} & =(-1)^{\operatorname{deg} x(\operatorname{deg} y+1)}\langle x, y\rangle_{\mathrm{PD}_{L(u)}} .
\end{aligned}
$$

Then $\left(H(L(\mathbf{u}) ; \Lambda),\langle\cdot, \cdot\rangle_{\text {cyc }}, \cup^{\mathfrak{c}, \mathfrak{b}, b}, \operatorname{PD}[L(\mathbf{u})]\right)$ becomes a unital Frobenius algebra.

REMARK 12.11. We remark that the operation $\mathfrak{m}_{2}^{\mathfrak{c}, \mathfrak{b}, b}$ is slightly different from the operation $\mathfrak{m}_{2}^{\mathfrak{b}, b}$ which is obtained from the operation $\mathfrak{q}_{\ell, k}$ by (36). In fact $\mathfrak{q}_{\ell, k}$ may not satisfy the cyclic symmetry:

$$
\begin{aligned}
& \left\langle\mathfrak{q}_{\ell ; k}\left(\mathbf{y} ; h_{1}, \ldots, h_{k}\right), h_{0}\right\rangle_{\text {сyc }} \\
& \quad=(-1)^{\operatorname{deg}^{\prime} h_{0}\left(\operatorname{deg}^{\prime} h_{1}+\cdots+\operatorname{deg}^{\prime} h_{k}\right)}\left\langle\mathfrak{q}_{\ell ; k}\left(\mathbf{y} ; h_{0}, h_{1}, \ldots, h_{k-1}\right), h_{k}\right\rangle_{\text {cyc. }}
\end{aligned}
$$

This is because the way how we perturb the moduli space $\mathcal{M}_{k+1 ; \ell}^{\text {main }}(\beta)$, which we described in sections 3 and 7 , breaks cyclic symmetry.

However we can modify the construction of $\mathfrak{q}_{\ell ; k}$ to obtain $\mathfrak{q}_{\ell ; k}^{\mathfrak{c}}$ for which (105) is satisfied. Using it in place of $\mathfrak{q}_{\ell ; k}$ we define $\mathfrak{m}_{2}^{\mathfrak{c}, \mathfrak{b}, b}$, which appears in (103). Then Definition 12.93 ) is satisfied for $\cup^{\mathfrak{b}, b}$.

This point is quite technical and delicate. So we do not discuss its detail in this survey and refer readers to [FOOO5] sections 3.2-3.3. However it is inevitable and essential, especially in the non-Fano case. It might be related to the fact that primitive form may be different from $d x_{1} \wedge \cdots \wedge d x_{n}$ in general. 
We put

$$
Z(\mathfrak{b}, b)=Z\left(\left(H(L(u) ; \Lambda),\langle\cdot, \cdot\rangle_{\mathrm{cyc}}, \cup^{\mathfrak{c}, \mathfrak{b}, b}, \operatorname{PD}([L(u)])\right) .\right.
$$

Definition 12.12. Assume that $\mathfrak{P O}^{\mathfrak{b}}$ is a Morse function. We then define a residue pairing

$$
\langle\cdot, \cdot\rangle_{\mathrm{res}}:\left(\operatorname{Jac}\left(\mathfrak{P O}^{\mathfrak{b}}\right) \otimes_{\Lambda_{0}} \Lambda\right) \otimes\left(\operatorname{Jac}\left(\mathfrak{P O}^{\mathfrak{b}}\right) \otimes_{\Lambda_{0}} \Lambda\right) \rightarrow \Lambda
$$

by

$$
\left\langle 1_{\mathfrak{y}}, 1_{\mathfrak{y}^{\prime}}\right\rangle_{\mathrm{res}}= \begin{cases}0 & \text { if } \mathfrak{y} \neq \mathfrak{y}^{\prime} \\ (Z(\mathfrak{b}, b))^{-1} & \text { if } \mathfrak{y}=\mathfrak{y}^{\prime}\end{cases}
$$

We remark that we use the decomposition in Proposition 11.8 and $1_{\mathfrak{y}}$ is the unit of $\operatorname{Jac}\left(\mathfrak{P O}^{\mathfrak{b}} ; \mathfrak{y}\right) \cdot \mathbf{u}=\left(u_{1}, \ldots, u_{n}\right)$ is defined by the valuation of $\mathfrak{y}=\left(\mathfrak{y}_{1}, \ldots, \mathfrak{y}_{n}\right)$. Namely $u_{i}=\mathfrak{v}_{T}\left(\mathfrak{y}_{i}\right) . b \in H^{1}\left(L(\mathbf{u}) ; \Lambda_{0}\right)$ is defined from $\mathfrak{y}_{i}$ by $b=\sum_{i=1}^{n} \mathfrak{x}_{i} \mathbf{e}_{i}, T^{u_{i}} e^{\mathfrak{x}_{i}}=\mathfrak{y}_{i}$.

The name 'residue pairing' is justified by the following Theorem 12.13 and Lemma 12.8.

THEOREM 12.13. 1) Assume that $\mathfrak{y}$ is a nondegenerate critical point of $\mathfrak{P O}^{\mathfrak{b}}$. Suppose $b=\sum_{i=1}^{n} \mathfrak{x}_{i} \mathbf{e}_{i}, T^{u_{i}} e^{\mathfrak{x}_{i}}=\mathfrak{y}_{i}$ as above. Then

$$
Z(\mathfrak{b}, b) \equiv \operatorname{det}\left[y_{i} y_{j} \frac{\partial^{2} \mathfrak{P} \mathfrak{O}^{\mathfrak{b}}}{\partial y_{i} \partial y_{j}}\right]_{i, j=1}^{i, j=n}(\mathfrak{y}) \bmod T^{\lambda} \Lambda_{+} .
$$

Here $\lambda=v_{T}(Z(\mathfrak{b}, b))$ and $\mathfrak{y}=\left(T^{u_{1}} e^{\mathfrak{x}_{1}}, \ldots, T^{u_{n}} e^{\mathfrak{x}_{n}}\right)$.

2) If $\operatorname{dim}_{\mathbb{C}} X=2$, then we have

$$
Z(\mathfrak{b}, b)=\operatorname{det}\left[y_{i} y_{j} \frac{\partial^{2} \mathfrak{P} \mathfrak{O}^{\mathfrak{c}, \mathfrak{b}}}{\partial y_{i} \partial y_{j}}\right]_{i, j=1}^{i, j=n}(\mathfrak{y}) .
$$

3) If $X$ is nef and $\operatorname{deg} \mathfrak{b}=2$, then we have

$$
Z(\mathfrak{b}, b)=\operatorname{det}\left[y_{i} y_{j} \frac{\partial^{2} \mathfrak{P \mathfrak { O } ^ { \mathfrak { b } }}}{\partial y_{i} \partial y_{j}}\right]_{i, j=1}^{i, j=n}(\mathfrak{y}) .
$$

REMARK 12.14. We use $\mathfrak{m}_{k}^{\mathfrak{c}, \mathfrak{b}}$ in place of $\mathfrak{m}_{k}^{\mathfrak{b}}$ to define $\mathfrak{P O}^{\mathfrak{c}, \mathfrak{b}}$ by

$$
\mathfrak{P O}^{\mathfrak{c}, \mathfrak{b}}(b)=\sum_{k=0}^{\infty} \int_{L(\mathbf{u})} \mathfrak{m}_{k}^{\mathfrak{c}, \mathfrak{b}}(\underbrace{b, \ldots, b}_{k}) .
$$

$\mathfrak{P O}^{\mathfrak{c}, \mathfrak{b}}$ appears in (109). 
Theorem 12.13 is Theorem 1.2.25 [FOOO5].

Sketch of the proof. We discuss only the case $X$ is nef and $\mathfrak{b}=$ 0. We will prove that the algebra $\left(H(L(u) ; \Lambda), \cup^{b}\right)$ is a Clifford algebra, modifying the proof of a related result by Cho [Cho2]. More precisely we prove the following Proposition 12.15.

Let $\mathbf{e}_{1}^{\prime}, \ldots, \mathbf{e}_{n}^{\prime}$ be formal variables and $d_{i} \in \Lambda \backslash\{0\}(i=1, \ldots, n)$. We consider relations

$$
\left\{\begin{aligned}
\mathbf{e}_{i}^{\prime} \mathbf{e}_{j}^{\prime}+\mathbf{e}_{j}^{\prime} \mathbf{e}_{i}^{\prime} & =0, \quad i \neq j \\
\mathbf{e}_{i}^{\prime} \mathbf{e}_{i}^{\prime} & =d_{i} 1 .
\end{aligned}\right.
$$

We take a free (non-commutative) $\Lambda$ algebra generated by $\mathbf{e}_{1}^{\prime}, \ldots, \mathbf{e}_{n}^{\prime}$ and divide it by the two-sided ideal generated by (111). We denote it by $\mathrm{Cliff}_{\Lambda}$ $(n ; \vec{d})$, where we set $\vec{d}=\left(d_{1}, \ldots, d_{n}\right)$.

Let $I=\left(i_{1}, \ldots, i_{k}\right), 1 \leq i_{1}<\cdots<i_{k} \leq n$. We write the set of such $I$ 's by $2^{\{1, \ldots, n\}}$. We put

$$
\mathbf{e}_{I}^{\prime}=\mathbf{e}_{i_{1}}^{\prime} \mathbf{e}_{i_{2}}^{\prime} \cdots \mathbf{e}_{i_{k-1}}^{\prime} \mathbf{e}_{i_{k}}^{\prime} \in \operatorname{Cliff}_{\Lambda}(n ; \vec{d}) .
$$

It is well-known and can be easily checked that $\left\{\mathbf{e}_{I}^{\prime} \mid I \in 2^{\{1, \ldots, n\}}\right\}$ forms a basis of $\operatorname{Cliff}_{\Lambda}(n ; \vec{d})$ as a $\Lambda$ vector space.

Assume moreover that there exists a $\Lambda$ valued non-degenerate inner product $\langle\cdot\rangle$ on $\operatorname{Cliff}_{\Lambda}(n ; \vec{d})$ such that $\operatorname{Cliff}_{\Lambda}(n ; \vec{d})$ becomes a Frobenius algebra. We say that $\mathbf{e}_{i}^{\prime}$ forms a cyclic Clifford basis if

$$
\left\langle\mathbf{e}_{I}^{\prime}, \mathbf{e}_{J}^{\prime}\right\rangle= \begin{cases}(-1)^{*(I)} & J=I^{c} \\ 0 & \text { otherwise. }\end{cases}
$$

Here $I^{c}=\{1, \ldots, n\} \backslash I$ and $*(I)=\#\left\{(i, j) \mid i \in I, j \in I^{c}, j<i\right\}$.

Proposition 12.15. Suppose $X$ is nef and $\operatorname{deg} \mathfrak{b}=2$. We also assume that $L(\mathbf{u})$ and $b \in H^{1}\left(L(\mathbf{u}) ; \Lambda_{0}\right)$ satisfy $H F((L(\mathbf{u}),(\mathfrak{b}, b)),(L(\mathbf{u}),(\mathfrak{b}, b)) ; \Lambda) \cong$ $H\left(T^{n} ; \Lambda\right)$.

Then there exists a basis $\left(\mathbf{e}_{1}^{\prime}, \ldots, \mathbf{e}_{n}^{\prime}\right)$ of $H^{1}(L(\mathbf{u}) ; \Lambda)$ such that the algebra $\left(\left(H(L(\mathbf{u}) ; \Lambda), \cup^{\mathfrak{c}, \mathfrak{b}, b}\right)\right.$ is isomorphic to the Clifford algebra $\operatorname{Cliff}_{\Lambda}(n ; \vec{d})$ where $\left(d_{1}, \ldots, d_{n}\right)$ satisfies

$$
{ }^{t} A\left[y_{i} y_{j} \frac{\partial^{2} \mathfrak{P O _ { \mathfrak { b } }}}{\partial y_{i} \partial y_{j}}(\mathfrak{y})\right]_{i, j=1}^{i, j=n} A=2\left[\begin{array}{cccc}
d_{1} & 0 & \ldots & 0 \\
0 & d_{2} & \ldots & 0 \\
\vdots & \vdots & \ddots & \vdots \\
0 & 0 & \ldots & d_{n}
\end{array}\right] .
$$

for a matrix $A$ with $\operatorname{det} A=1$.

Moreover $\left(\mathbf{e}_{1}^{\prime}, \ldots, \mathbf{e}_{n}^{\prime}\right)$ is a cyclic Clifford basis.

Furthermore

$$
\int_{L(\mathbf{u})} \mathbf{e}_{1}^{\prime} \cup^{\mathfrak{b}, b} \cdots \cup^{\mathfrak{b}, b} \mathbf{e}_{n}^{\prime}=1
$$


This is [FOOO5] Theorem 3.6.2. Once Proposition 12.15 is established we can prove Theorem 12.13 by a direct calculation. (See [FOOO5] section 3.7)

Sketch of the proof of Proposition 3.4.8. Note

$$
\mathfrak{P O}^{\mathfrak{b}}(b)=\sum_{k=0}^{\infty} \int_{L(\mathbf{u})} \mathfrak{m}_{k}^{\mathfrak{b}}(\underbrace{b, \ldots, b}_{k}) .
$$

Its first derivative at $\mathfrak{y}$ is zero since $\mathfrak{y}$ is a critical point. We calculate its second derivative $\partial^{2} \mathfrak{P O}^{\mathfrak{b}} / \partial x_{i} \partial x_{j}=y_{i} y_{j} \partial^{2} \mathfrak{P} \mathfrak{O}^{\mathfrak{b}} / \partial y_{i} \partial y_{j}$. Then we have

$$
\mathfrak{m}_{2}^{\mathfrak{b}, b}\left(\mathbf{e}_{i}, \mathbf{e}_{j}\right)+\mathfrak{m}_{2}^{\mathfrak{b}, b}\left(\mathbf{e}_{j}, \mathbf{e}_{i}\right)=\left(\left(y_{i} y_{j} \frac{\partial^{2} \mathfrak{P} \mathfrak{O}^{\mathfrak{b}}}{\partial y_{i} \partial y_{j}}\right)(\mathfrak{y})\right) 1
$$

Here $1 \in H^{0}(L(\mathbf{u}) ; \mathbb{Q})$ is the unit and $\left\{\mathbf{e}_{i}\right\}$ is the basis of $H^{1}(L(\mathbf{u}) ; \mathbb{Q})$ which we fixed before. (Note $b=\sum x_{i} \mathbf{e}_{i}$.)

We take basis $\left(\mathbf{e}_{1}^{\prime}, \ldots, \mathbf{e}_{n}^{\prime}\right)$ of $H^{1}(L(\mathbf{u}) ; \Lambda)$ so that the quadratic form associated to the Hessian matrix becomes diagonal and $\int_{L(\mathbf{u})} \mathbf{e}_{1}^{\prime} \cup \cdots \cup \mathbf{e}_{n}^{\prime}=1$. Then (113) implies that $\left(\mathbf{e}_{1}^{\prime}, \ldots, \mathbf{e}_{n}^{\prime}\right)$ satisfies the Clifford relation (111). Using this fact we can prove that $\left(\left(H(L(\mathbf{u}) ; \Lambda), \cup^{\mathfrak{c}, \mathfrak{b}, b}\right)\right.$ is a Clifford algebra. (We do not use the assumption $X$ is nef and $\mathfrak{b}$ is degree two, up to this point.)

The proof of (112) is as follow. We use the assumption that $X$ is nef and $\mathfrak{b}$ is degree two to show

$$
\mathfrak{a} \cup^{\mathfrak{c}, \mathfrak{b}, b} \mathfrak{a}^{\prime}-\mathfrak{a} \cup \mathfrak{a}^{\prime} \in \bigoplus_{k<\operatorname{deg} \mathfrak{a}+\operatorname{deg} \mathfrak{a}^{\prime}} H^{k}\left(T^{n} ; \Lambda\right)
$$

for $\mathfrak{a}, \mathfrak{a}^{\prime} \in H F((L(\mathbf{u}),(\mathfrak{b}, b)),(L(\mathbf{u}),(\mathfrak{b}, b)) ; \Lambda) \cong H\left(T^{n} ; \Lambda\right)$. Here the second term is the usual cup product. We use cyclic symmetry to show

$$
\left\langle\mathbf{e}_{I}^{\prime}, \mathbf{e}_{J}^{\prime}\right\rangle_{\mathrm{PD}_{L(\mathbf{u})}}=\left\langle\mathbf{e}_{I}^{\prime} \cup^{\mathfrak{c}, \mathfrak{b}, b} \mathbf{e}_{J}^{\prime}, 1\right\rangle_{\mathrm{PD}_{L(\mathbf{u})}}=\int_{L(\mathbf{u})} \mathbf{e}_{I}^{\prime} \cup^{\mathfrak{c}, \mathfrak{b}, b} \mathbf{e}_{J}^{\prime}
$$

Using (114) and the Clifford relation, we can see that $\mathbf{e}_{I}^{\prime} \cup^{\mathfrak{c}, \mathfrak{b}, b} \mathbf{e}_{J}^{\prime}$ has no $H^{n}(L(\mathbf{u}) ; \Lambda)$ component unless $I^{c}=J$. This implies Proposition 12.15.

\subsection{Residue pairing is Poincaré duality.}

TheOREM 12.16. Let $X$ be a compact toric manifold and $\mathfrak{b} \in \mathcal{A}\left(\Lambda_{0}\right)$. Suppose $\mathfrak{P D}^{\mathfrak{b}}$ is a Morse function. Then for each $\mathfrak{a}_{1}, \mathfrak{a}_{2} \in H(X ; \Lambda)$ we have

$$
\left\langle\mathfrak{a}_{1}, \mathfrak{a}_{2}\right\rangle_{\mathrm{PD}_{X}}=\left\langle\mathfrak{k}_{\mathfrak{S}_{\mathfrak{b}}} \mathfrak{a}_{1}, \mathfrak{k}_{\mathfrak{S}_{\mathfrak{b}}} \mathfrak{a}_{2}\right\rangle_{\mathrm{res}} .
$$

Here the pairing in the right hand side is defined in Definition 12.12 and the map $\mathfrak{k}_{\mathfrak{s}_{\mathfrak{b}}}$ is the isomorphism in Theorem 11.14. The pairing in the left hand side is the Poincaré duality. 
Theorem 12.16 is [FOOO5] Theorem 1.1.1 (2) and is proved in [FOOO5] Chapter 3. Before explaining an outline of its proof, we mention some of its consequences.

Corollary $12.17 . \quad 1)$ The inner product $\langle\cdot\rangle_{\mathrm{res}}$, whose definition was given only in case $\mathfrak{P O}^{\mathfrak{b}}$ is a Morse function (in Definition 12.12), extends to arbitrary $\mathfrak{b}$ 's.

2) The Levi-Civita connection $\nabla$ of this extended $\langle\cdot\rangle_{\mathrm{res}}$ is flat.

3) $\left(H\left(X ; \Lambda_{0}\right),\langle\cdot\rangle_{\mathrm{res}}, \nabla, \circ, \Phi, 1\right)$ is a Frobenius manifold.

4) The Frobenius manifold structure of Item 3) above is equal to one in Theorem 12.4.

Proof. 1) is an immediate consequence of Theorem 12.16 and the fact that the Poincaré duality pairing is independent of $\mathfrak{b}$ and is obviously extended.

The Levi-Civita connection of the Poincaré duality pairing is the canonical affine connection of $H\left(X ; \Lambda_{0}\right)$ and hence is flat. 2) follows.

3) then follows from Theorem 12.4.

4) is obvious.

REMARK 12.18. The Frobenius manifold in Corollary 12.17 3) has an Euler vector field (96) with $r_{i}=1$. We also have

$$
\mathfrak{E}(\mathfrak{P O})=\mathfrak{P O}
$$

here $\mathfrak{P O}$ is a function of $\mathfrak{b}=\sum w_{i} \mathbf{p}_{i}$ and $y_{i}$. The formula (116) is proved in [FOOO4] Theorem 10.2.

REMARK 12.19. Corollary 12.17 first appeared as a conjecture in [Ta], where the case of $\mathbb{C} P^{1}$ was checked. It was further studied in [Bar]. See the papers mentioned at the end of the introduction for some of the other related works.

The above proof of the coincidense of the two Frobenius manifold structures is not so satisfactory since the proof of Items 1), 2) uses the isomorphism of Item 4). It is preferable that we construct Frobenius manifold structure on $H\left(X ; \Lambda_{0}\right)$ using the family of functions $\mathfrak{P O}^{\mathfrak{b}}$ and without going to the quantum cohomology theory side, and then prove Item 4) for that Frobenius manifold structure.

Problem 12.20. Develop an analogue of K. Saito theory for our family of $\Lambda$ valued functions $\mathfrak{P O}^{\mathfrak{b}}$.

Define the notion of primitive form for it and prove its existence.

Construct the Frobenius manifold structure on $H\left(X ; \Lambda_{0}\right)$ using primitive form and prove that it is isomorphic to one obtained in Theorem 12.4. 
Another corollary of Theorem 12.16 is the following. Let $\operatorname{Crit}\left(\mathfrak{P O}^{\mathfrak{b}}\right)$ be the critical point set of $\mathfrak{P} \mathfrak{O}^{\mathfrak{b}}$. For $\mathfrak{y}=\left(\mathfrak{y}_{1}, \ldots, \mathfrak{y}_{n}\right) \in \mathfrak{A}(P)$ we put

$$
\mathfrak{y}_{i}=T^{u_{i}} e^{\mathfrak{x}_{i}}, \quad b=\sum_{i=1}^{n} \mathfrak{x}_{i} \mathbf{e}_{i} \in H^{1}\left(L(\mathbf{u}), \Lambda_{0}\right) .
$$

Here $\mathbf{u}=\left(u_{1}, \ldots, u_{n}\right) \in P$ and $\mathfrak{x}_{i} \in \Lambda_{0}$. Note $u_{i}=\mathfrak{v}_{T}\left(\mathfrak{y}_{i}\right)$. In this way we may regard $\operatorname{Crit}\left(\mathfrak{P O}^{\mathfrak{b}}\right)$ as a set of pairs $\left(\mathbf{u}_{c}, b_{c}\right), c=1, \ldots, B^{\prime}$. Here we put $B^{\prime}=$ $\# \operatorname{Crit}\left(\mathfrak{P O}^{\mathfrak{b}}\right)$.

Corollary 12.21. Suppose $\mathfrak{P O}^{\mathfrak{b}}$ is a Morse function. Then we have

$$
0=\sum_{c=1}^{B^{\prime}} \frac{1}{Z\left(\mathfrak{b}, b_{c}\right)}
$$

Proof. Let $1_{X} \in H^{0}(X ; \Lambda)$ be the unit. Then $\left\langle 1_{X}, 1_{X}\right\rangle_{\mathrm{PD}_{X}}=0$. By Proposition 11.8 we have $1_{X}=\sum_{\mathfrak{y} \in \mathfrak{M}(X, \mathfrak{b})} 1_{\mathfrak{y}}$ where $1_{\mathfrak{y}}$ is the unit of the Jacobian ring $\operatorname{Jac}\left(\mathfrak{P O}_{\mathfrak{b}} ; \mathfrak{y}\right)$. Corollary 12.21 now follows from (107) and Theorem 12.16 .

12.5. Operator $\mathfrak{p}$ and the Poincaré dual to $\mathfrak{k}_{\mathfrak{b}}$. In this and the next subsections we sketch a proof of Theorem 12.16. We assume $\mathfrak{P O}^{\mathfrak{b}}$ is a Morse function in this and next subsections. Let $\mathfrak{y} \in \operatorname{Crit}\left(\mathfrak{P O}^{\mathfrak{b}}\right)$. It defines $\mathbf{u}, b$ by (117). We define a homomorphism

$$
i_{\mathrm{qm},(\mathfrak{b}, b, \mathbf{u})}^{*}: H\left(X ; \Lambda_{0}\right) \rightarrow H F\left((L(\mathbf{u}),(\mathfrak{b}, b)) ;(L(\mathbf{u}),(\mathfrak{b}, b)) ; \Lambda_{0}\right)
$$

by

$$
i_{\mathrm{qm},(\mathfrak{b}, b, \mathbf{u})}^{*}(Q)=\sum_{k=0}^{\infty} \sum_{\ell_{1}=0}^{\infty} \sum_{\ell_{2}=0}^{\infty} \mathfrak{q}_{\ell_{1}+\ell_{2} ; k}^{\mathfrak{c}}\left(\mathfrak{b}^{\ell_{1}} Q \mathfrak{b}^{\ell_{2}}, b^{k}\right) .
$$

(See (78) and [FOOO1] Theorem 3.8.62.)

Here $\mathfrak{q}_{\ell ; k}^{\mathfrak{c}}$ is a cyclically symmetric version of the operator $\mathfrak{q}_{\ell ; k}$. (See Remark 12.14.)

We define

$$
i_{*, \mathrm{qm},(\mathfrak{b}, b, \mathbf{u})}: H F((L(\mathbf{u}), \mathfrak{b}, b) ;(L(\mathbf{u}), \mathfrak{b}, b) ; \Lambda) \rightarrow H(X ; \Lambda)
$$

by

$$
\left\langle i_{\mathrm{qm},(\mathfrak{b}, b, \mathbf{u})}^{*}(Q), P\right\rangle_{\mathrm{PD}_{L(\mathbf{u})}}=\left\langle Q, i_{*, \mathrm{qm},(\mathfrak{b}, b, \mathbf{u})}(P)\right\rangle_{\mathrm{PD}_{X}} .
$$

The main part of the proof of Theorem 12.16 is the proof of Theorem 12.22 below. Let $\operatorname{vol}_{L(\mathbf{u})} \in H^{n}(X ; \mathbb{Q})$ be the degree $n$ cohomology class such that $\int_{L(\mathbf{u})} \operatorname{vol}_{L(\mathbf{u})}=1$. Let $\left\{\mathbf{e}_{I} \mid I \in 2^{n}\right\}$ be a basis of

$$
H^{n}(L(\mathbf{u}) ; \Lambda) \cong H F((L(\mathbf{u}),(\mathfrak{b}, b)) ;(L(\mathbf{u}),(\mathfrak{b}, b)) ; \Lambda) .
$$

We put $g_{I J}=\left\langle\mathbf{e}_{I}, \mathbf{e}_{J}\right\rangle_{P D_{X}}$. Let $g^{I J}$ be the inverse matrix of $g_{I J}$. 
TheOREM 12.22. We have:

$$
\begin{aligned}
& \left\langle i_{*, \mathrm{qm},(\mathfrak{b}, b, \mathbf{u})}\left(\operatorname{vol}_{L(\mathbf{u})}\right), i_{*, \mathrm{qm},(\mathfrak{b}, b, \mathbf{u})}\left(\operatorname{vol}_{L(\mathbf{u})}\right)\right\rangle_{\mathrm{PD}_{X}} \\
& \quad=\sum_{I, J \in 2^{\{1, \ldots, n\}}}(-1)^{\frac{n(n-1)}{2}} g^{I J}\left\langle\mathfrak{m}_{2}^{\mathfrak{c}, \mathfrak{b}, b}\left(\mathbf{e}_{I}, \operatorname{vol}_{L(\mathbf{u})}\right), \mathfrak{m}_{2}^{\mathfrak{c}, \mathfrak{b}, b}\left(\mathbf{e}_{J}, \operatorname{vol}_{L(\mathbf{u})}\right)\right\rangle_{\mathrm{PD}_{L(u)}} .
\end{aligned}
$$

This is [FOOO5] Theorem 3.4.1.

Theorem $12.22 \Rightarrow$ Theorem 12.16. Let $Q_{\mathfrak{n}} \in H(X ; \Lambda)$ be an element such that $\mathfrak{k}_{\mathfrak{b}}\left(Q_{\mathfrak{y}}\right)=1_{\mathfrak{y}}$, where $1_{\mathfrak{y}}$ is the unit of the factor $\operatorname{Jac}\left(\mathfrak{P} \mathfrak{O}^{\mathfrak{b}} ; \mathfrak{y}\right)$ of $\operatorname{Jac}\left(\mathfrak{P O}^{\mathfrak{b}}\right)$. Let $b, \mathbf{u}$ corresponds to $\mathfrak{y}$ by $(117)$.

Then we have

$$
i_{\mathrm{qm},(\mathfrak{b}, b, \mathbf{u})}^{*}\left(Q_{\mathfrak{y}^{\prime}}\right)= \begin{cases}1 & \text { if } \mathfrak{y}^{\prime}=\mathfrak{y} \\ 0 & \text { if } \mathfrak{y}^{\prime} \neq \mathfrak{y} .\end{cases}
$$

Here $1 \in H^{0}(L(\mathbf{u}) ; \Lambda)$ is the unit. This is a consequence of the definition of $\mathfrak{k}_{\mathfrak{b}}$. Therefore

$$
\left\langle Q_{\mathfrak{y}}, i_{*, \mathrm{qm},(\mathfrak{b}, b, \mathbf{u})}\left(\operatorname{vol}_{L(\mathbf{u})}\right)\right\rangle_{\mathrm{PD}_{X}}=1 .
$$

We remark $\left\langle Q_{\mathfrak{y}}, Q_{\mathfrak{y}^{\prime}}\right\rangle=\left\langle Q_{\mathfrak{y}} \cup^{\mathfrak{b}} Q_{\mathfrak{y}^{\prime}}, 1\right\rangle=0$ if $\mathfrak{y} \neq \mathfrak{y}^{\prime}$. Therefore

$$
i_{*, \mathrm{qm},(\mathfrak{b}, b, \mathbf{u})}\left(\operatorname{vol}_{L(u)}\right)=\frac{1}{\left\langle Q_{\mathfrak{y}}, Q_{\mathfrak{y}}\right\rangle_{\mathrm{PD}_{X}}} Q_{\mathfrak{y}}
$$

Theorem 12.22 implies

$$
\left\langle i_{*, \mathrm{qm},(\mathfrak{b}, b, \mathbf{u})}\left(\operatorname{vol}_{L(\mathbf{u})}\right), i_{*, \mathrm{qm},(\mathfrak{b}, b, \mathbf{u})}\left(\operatorname{vol}_{L(\mathbf{u})}\right)\right\rangle_{\mathrm{PD}_{X}}=Z(\mathfrak{b}, b) .
$$

(See [FOOO5] subsection 3.10.2 for sign.) Theorem 12.16 follows from (124) and (125).

To prove Theorem 12.22 we need a geometric description of the homomorphism $i_{*, \mathrm{qm},(\mathfrak{b}, b, \mathbf{u})}$. We use the operator $\mathfrak{p}$ introduced in [FOOO1] section 3.8 , for this purpose. To simplify the notation we consider only the case $\mathfrak{b}=\mathbf{0}$. Let $C$ be a filtered $A_{\infty}$ algebra and define an automorphism cyc : $B_{k} C[1] \rightarrow B_{k} C[1]$ by

$$
\operatorname{cyc}\left(x_{1} \otimes \cdots \otimes x_{k}\right)=(-1)^{\operatorname{deg}^{\prime} x_{k} \times\left(\sum_{i=1}^{k-1} \operatorname{deg}^{\prime} x_{i}\right)} x_{k} \otimes x_{1} \otimes \cdots \otimes x_{k-1} .
$$

It induces a $\mathbb{Z}_{k}$ action on $B_{k} C[1]$. Let $B_{k}^{\text {cyc }} C[1]$ be the invariant set of the $\mathbb{Z}_{k}$ action and $B^{\mathrm{cyc}} C[1]=\widehat{\bigoplus}_{k} B_{k}^{\mathrm{cyc}} C[1]$ the completed direct sum of them. We call $B_{k}^{\text {cyc }} C[1]$ the cyclic bar complex.

THEOREM 12.23. For a relatively spin Lagrangian submanifold $L$ there exists a sequence of operators

$$
\mathfrak{p}_{k}: B_{k}^{\text {cyc }} H\left(L ; \Lambda_{0}\right)[1] \longrightarrow H\left(X ; \Lambda_{0}\right)
$$

$(k=0,1,2, \ldots)$ of degree $n+1$ with the following properties. 
Let $\mathfrak{p}: B^{\mathrm{cyc}} H\left(L ; \Lambda_{0}\right)[1] \longrightarrow H\left(X ; \Lambda_{0}\right)$ be the operator whose restriction on $B_{k}^{\text {cyc }} H\left(L ; \Lambda_{0}\right)[1]$ is $\mathfrak{p}_{k}$. We denote by $\mathfrak{m}_{k}^{\mathfrak{c}}$ the cyclically symmetric version of $\mathfrak{m}_{k}$ and write $\mathfrak{m}^{\mathfrak{c}}$ instead of $\mathfrak{m}_{k}^{\mathfrak{c}}$.

1)

$$
\mathfrak{p}_{1} \equiv i_{!} \quad \bmod \Lambda_{+}
$$

Here $i_{!}=H^{k}\left(L ; \Lambda_{0}\right) \rightarrow H^{k+n}(X ; \Lambda)$ is the Gysin homomorphism.

2)

$$
\sum_{c} \mathfrak{p}\left(\mathbf{x}_{c}^{3 ; 1} \otimes \mathfrak{m}^{\mathfrak{c}}\left(\mathbf{x}_{c}^{3 ; 2}\right) \otimes \mathbf{x}_{c}^{3 ; 3}\right)=0
$$

3)

for $\mathbf{x} \in B_{k}^{\text {cyc }} H\left(L ; \Lambda_{0}\right)[1], k>0$. We use the notation (6).

$$
\left(\mathfrak{p}_{1} \circ \mathfrak{m}_{0}^{\mathfrak{c}}\right)(1)+G W_{1}(L)=0 .
$$

Here the second term is defined by $\left\langle G W_{1}(L), Q\right\rangle_{\mathrm{PD}_{X}}=G W_{2}(L, Q)$, where the right hand side is as in (68).

This is [FOOO1] Theorem 3.8.9. (Here we use cohomology group instead of appropriate chain complex. The latter is used in [FOOO1] Theorem 3.8.9. We also omit the statement on the unit in [FOOO1] Theorem 3.8.9.) See also [FOOO5] section 3.1-3.3.

The operator $\mathfrak{p}_{k}$ is constructed as follows. We consider the moduli space $\mathcal{M}_{k ; 1}^{\operatorname{main}}(\beta)$ described in section 2.2. Note the number of interior marked point is 1 and the number of exterior marked points is $k$. We have an evaluation map

$$
\left(\mathrm{ev}_{1}, \ldots, \mathrm{ev}_{k}, \mathrm{ev}^{+}\right)=\left(\mathrm{ev}, \mathrm{ev}^{+}\right): \mathcal{M}_{k ; 1}^{\operatorname{main}}(\beta) \rightarrow L^{k} \times X .
$$

Let $h_{1}, \ldots, h_{k}$ be differential forms on $L$. We consider the pull back ev* $\left(h_{1} \times\right.$ $\cdots \times h_{k}$ ), which is a differential form on $\mathcal{M}_{k ; 1}^{\operatorname{main}}(\beta)$. We use integration along fiber by the map $\mathrm{ev}^{+}$to obtain a differential form on $X$, which we put $\mathfrak{p}_{k, \beta}\left(h_{1}, \ldots, h_{k}\right)$. Namely

$$
\mathfrak{p}_{k, \beta}\left(h_{1}, \ldots, h_{k}\right)=\mathrm{ev}_{!}^{+}\left(\mathrm{ev}^{*}\left(h_{1} \times \cdots \times h_{k}\right)\right) .
$$

This is a map between differential forms. By an algebraic argument it induces a map between tensor products of the de Rham cohomology groups of $L$ and of $X$. Thus obtain the operator

$$
\mathfrak{p}_{k}=\sum_{\beta \in H_{2}(X, L)} T^{(\beta \cap \omega) / 2 \pi} \mathfrak{p}_{k, \beta} .
$$

We can prove (126) by studying the stable map compactification of $\mathcal{M}_{k ; 1}^{\text {main }}(\beta)$. In case $k=0$ the compactification of $\mathcal{M}_{0 ; 1}(\beta)$ is slightly different from the case of $k>0$. The second term of Item 3) appears by this reason. In our case of toric manifold and $T^{n}$ orbit $L$, this term drops since $L$ is homologous to 0 in $X$. So we do not discuss it here but refer to [FOOO1] subsections 3.8.3 and 7.4.1 for more detail. 
Now we go back to the case where $X$ is a toric manifold and $L=L(\mathbf{u})$ is a $T^{n}$ orbit. Let $b \in H^{1}\left(L(\mathbf{u}) ; \Lambda_{0}\right)$. For $P \in H\left(L(\mathbf{u}) ; \Lambda_{0}\right)$ we put

$$
\left[P e^{b}\right]=\sum_{k_{1}=0}^{\infty} \sum_{k_{2}=0}^{\infty} \underbrace{b \otimes \cdots \otimes b}_{k_{1}} \otimes P \otimes \underbrace{b \otimes \cdots \otimes b}_{k_{2}} .
$$

Suppose $H(L(\mathbf{u}) ; \Lambda) \cong H F((L(\mathbf{u}),(\mathbf{0}, b)) ;(L(\mathbf{u}),(\mathbf{0}, b)) ; \Lambda)$.

Proposition 12.24. Let $P \in H\left(L(\mathbf{u}) ; \Lambda_{0}\right)$. Then we have:

$$
i_{*, \mathrm{qm},(\mathbf{0}, b, \mathbf{u})}(P)=\mathfrak{p}\left(\left[P e^{b}\right]\right) .
$$

REMARK 12.25. We remark that $\left[P e^{b}\right]$ is an element of $B^{\text {cyc }} H\left(L(\mathbf{u}) ; \Lambda_{0}\right)$ if $b \equiv 0 \bmod \Lambda_{+}$. So $\mathfrak{p}\left(\left[P e^{b}\right]\right)$ is defined in that case. Otherwise we write $b=b_{0}+b_{+}$such that $b_{0} \in H^{1}(L(\mathbf{u}) ; \mathbb{C})$ and $b_{+} \in H^{1}\left(L(\mathbf{u}) ; \Lambda_{+}\right)$, and define

$$
\mathfrak{p}\left(\left[P e^{b}\right]\right)=\sum_{\beta \in H_{2}(X, L: \mathbb{Z})} T^{(\beta \cap \omega) / 2 \pi} \exp \left(b_{0} \cap \partial \beta\right) \mathfrak{p}_{\beta}\left(\left[P e^{b_{+}}\right]\right) .
$$

We omit the discussion of this point. See [FOOO4] section 9 and [FOOO5] section 3.3.

Sketch of The PRoOF. Let $Q \in H\left(X ; \Lambda_{0}\right)$. We remark that $i_{*, \mathrm{qm},(0, b, \mathbf{u})}(P)$ is defined by (122). Therefore it suffices to prove

$$
\sum_{k=0}^{\infty}\left\langle\mathfrak{q}_{1, k}^{\mathfrak{c}}\left(Q ; b^{k}\right), P\right\rangle_{\mathrm{PD}_{L(\mathbf{u})}}=\left\langle Q, \mathfrak{p}\left(\left[P e^{b}\right]\right)\right\rangle_{\mathrm{PD}_{X}} .
$$

This is [FOOO5] Theorem 19.8. Let us sketch its proof for the case $b=0$. In case $b=0$, Formula (128) reduced to

$$
\left\langle\mathfrak{q}_{1,0}^{\mathfrak{c}}(Q ; 1), P\right\rangle_{\mathrm{PD}_{L(\mathbf{u})}}=\left\langle Q, \mathfrak{p}_{1}(P)\right\rangle_{\mathrm{PD}_{X}}
$$

We take $\rho$ and $h$ which are closed forms on $X$ and $L(\mathbf{u})$, representing the cohomology class $Q$ and $P$, respectively. Then it is easy to see that the left and the right hand sides of (129) both become

$$
\sum_{\beta \in H_{2}(X, L(\mathbf{u}) ; \mathbb{Z})} T^{(\beta \cap \omega) / 2 \pi} \int_{\mathcal{M}_{1 ; 1}(\beta)}\left(\mathrm{ev}^{+}\right)^{*} \rho \wedge \mathrm{ev}^{*} h .
$$

Here $\left(\mathrm{ev}, \mathrm{ev}^{+}\right): \mathcal{M}_{1 ; 1}(\beta) \rightarrow L(\mathbf{u}) \times X$ is evaluation maps at marked points. (129) follows.

REMARK 12.26. In fact, we need to perturb $\mathcal{M}_{1 ; 1}(\beta)$ appropriately so that the integration in (130) makes sense. It is a nontrivial thing to prove that after perturbation (129) still holds. Actually we need to consider cyclically symmetric version of the operator $\mathfrak{q}$ for this purpose. (See [FOOO5] Remark 3.3.12.) We omit the discussion about perturbation and refer the reader to [FOOO5] section 3.3. 
12.6. Annulus argument. We continue the sketch of the proof of Theorem 12.22. We assume $\mathfrak{b}=\mathbf{0}$ in this subsection for simplicity. We consider the class $\operatorname{vol}_{L(\mathbf{u})}$. (It is the Poincaré dual to the point class.) Then the left hand side is

$$
\sum_{\substack{\beta_{1}, \beta_{2} \in H_{2}(X, L(\mathbf{u}) ; \mathbb{Z}), \beta=\beta_{1}+\beta_{2}}} T^{\left(\left(\beta_{1}+\beta_{2}\right) \cap \omega\right) / 2 \pi}\left\langle\mathfrak{p}_{\beta_{1}}\left(\left[\operatorname{vol}_{L(\mathbf{u})} e^{b}\right]\right), \mathfrak{p}_{\beta_{2}}\left(\left[\operatorname{vol}_{L(\mathbf{u})} e^{b}\right]\right)\right\rangle_{\mathrm{PD}_{X}} .
$$

We show that (131) can be regarded as an appropriate integration of the differential form $\operatorname{vol}_{L(\mathbf{u})} \times \operatorname{vol}_{L(\mathbf{u})}$ on a moduli space of pseudo-holomorphic annuli, as follows. For simplicity we assume $b=0$.

We consider a pair $\left(\left(\Sigma ; z_{1}, z_{2}\right), u\right)$ with the following properties.

1) $\Sigma$ is a bordered curve of genus zero such that $\partial \Sigma$ is a disjoint union of two circles, which we denote by $\partial_{1} \Sigma, \partial_{2} \Sigma$.

2) The singularity of $\Sigma$ is at worst the double point.

3) $z_{i} \in \partial_{i} \Sigma$ for $i=1,2$.

4) $u: \Sigma \rightarrow X$ is a pseudo-holomorphic map. $u(\partial \Sigma) \subset L(\mathbf{u})$.

5) $u_{*}([\Sigma])=\beta \in H_{2}(X, L(\mathbf{u}) ; \mathbb{Z})$.

6) The set of maps $v: \Sigma \rightarrow \Sigma$ which is biholomorphic, $v\left(z_{i}\right)=z_{i}$ for $i=1,2$, and $u \circ v=u$ is finite.

We denote by $\mathcal{M}_{(1,1) ; 0}(\beta)$ the totality of such $\left(\left(\Sigma ; z_{1}^{+}, z_{2}^{+}\right), u\right)$. There exists an evaluation map

$$
\mathrm{ev}=\left(\mathrm{ev}_{1}, \mathrm{ev}_{2}\right): \mathcal{M}_{(1,1) ; 0}(\beta) \rightarrow L(\mathbf{u})^{2},
$$

which is defined by

$$
\mathrm{ev}\left(\left(\Sigma ; z_{1}, z_{2}\right), u\right)=\left(u\left(z_{1}\right), u\left(z_{2}\right)\right) .
$$

We consider the set of all $\left(\Sigma ; z_{1}, z_{2}\right)$ which satisfies 1$\left.\left.), 2\right), 3\right)$ above and

7) The set of all biholomorphic maps $v: \Sigma \rightarrow \Sigma$ with $v\left(z_{i}\right)=z_{i}$ for $i=1,2$ is finite.

We denote it by $\mathcal{M}_{(1,1) ; 0}$. There is a forgetful map

$$
\text { forget }: \mathcal{M}_{(1,1) ; 0}(\beta) \rightarrow \mathcal{M}_{(1,1) ; 0},
$$

which is obtained by forgetting the map $u$.

We can show that $\mathcal{M}_{(1,1) ; 0}$ is homeomorphic to a disk and so is connected. We take two points $\left(\Sigma^{(j)} ; z_{1}^{(j)}, z_{2}^{(j)}\right) \in \mathcal{M}_{(1,1) ; 0}(j=1,2)$ which we show in the figure below.

We denote by $\mathcal{M}_{(1,1) ; 0}\left(\beta ; \Sigma^{(j)}\right)$ the inverse image of $\left\{\left(\Sigma^{(j)} ; z_{1}^{(j)}, z_{2}^{(j)}\right)\right\}$ by the map (132). 


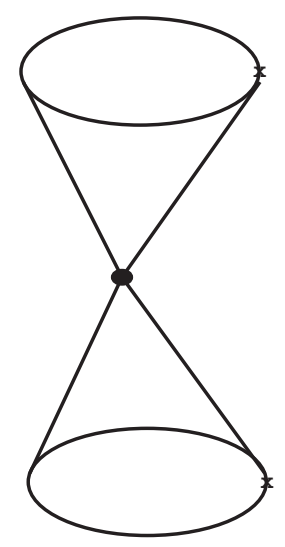

$\Sigma^{(1)}$

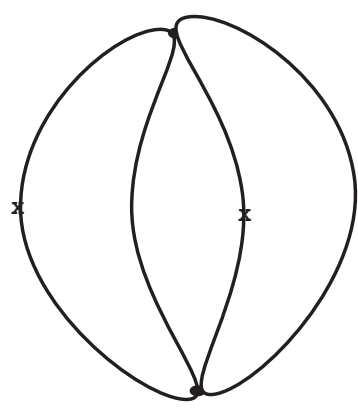

$\Sigma^{(2)}$

FIGURE 12.2

LEMMA 12.27 .

$$
\begin{aligned}
& \quad \sum_{\substack{\beta_{1}, \beta_{2} \in H_{2}(X, L(\mathbf{u}) ; Z), \beta=\beta_{1}+\beta_{2}}}\left\langle\mathfrak{p}_{1, \beta_{1}}\left(\operatorname{vol}_{L(\mathbf{u})}\right), \mathfrak{p}_{1, \beta_{2}}\left(\operatorname{vol}_{L(\mathbf{u})}\right)\right\rangle_{\mathrm{PD}_{X}} \\
& =\int_{\mathcal{M}_{(1,1) ; 0}\left(\beta ; \Sigma^{(1)}\right)} \mathrm{ev}_{1}^{*} \operatorname{vol}_{L(\mathbf{u})} \wedge \mathrm{ev}_{2}^{*} \operatorname{vol}_{L(\mathbf{u})} .
\end{aligned}
$$

Geometric origin of this lemma is clear from Figure 12.2. To prove the lemma rigorously we need to work out the way to perturb our moduli space $\mathcal{M}_{(1,1) ; 0}\left(\beta ; \Sigma^{(1)}\right)$ so that the integration of the right hand side makes sense and the lemma holds. The detail is given in [FOOO5] section 3.4 as the proof of Proposition 3.4.8.

LEMMA 12.28 .

$$
\begin{aligned}
& \quad \sum_{I, J \in 2^{\{1, \ldots, n\}}}(-1)^{\frac{n(n-1)}{2}} g^{I J}\left\langle\mathfrak{m}_{2}^{\mathfrak{c}, 0,0}\left(\mathbf{e}_{I}, \operatorname{vol}_{L(\mathbf{u})}\right), \mathfrak{m}_{2}^{\mathfrak{c}, 0,0}\left(\mathbf{e}_{J}, \operatorname{vol}_{L(\mathbf{u})}\right)\right\rangle_{\mathrm{PD}_{L(u)}} \\
& =\int_{\mathcal{M}_{(1,1) ; 0}\left(\beta ; \Sigma^{(2)}\right)} \operatorname{ev}_{1}^{*} \operatorname{vol}_{L(\mathbf{u})} \wedge \mathrm{ev}_{2}^{*} \operatorname{vol}_{L(\mathbf{u})} .
\end{aligned}
$$

Geometric origin of this lemma is also clear from Figure 12.2 and the equality

$$
\begin{aligned}
{[\{(x, x) \mid x \in L(\mathbf{u})\}] } & =\sum_{I, J}(-1)^{\operatorname{deg} \mathbf{e}_{I} \operatorname{deg} \mathbf{e}_{J}} g^{I J} \mathbf{e}_{I} \times \mathbf{e}_{J} \\
& \in H_{n}(L(\mathbf{u}) \times L(\mathbf{u}) ; \mathbb{Z}) .
\end{aligned}
$$

The detail is given in [FOOO5] section 3.4 as the proof of Proposition 3.4.10. (The sign in (133) is proved in [FOOO5] Lemma 3.10.7.) 
Now we can use the fact that $\mathcal{M}_{(1,1) ; 0}$ is connected to find a cobordism between $\mathcal{M}_{(1,1) ; 0}\left(\beta ; \Sigma^{(1)}\right)$ and $\mathcal{M}_{(1,1) ; 0}\left(\beta ; \Sigma^{(2)}\right)$. The differential form $\mathrm{ev}_{1}^{*} \mathrm{vol}_{L(\mathbf{u})} \wedge \mathrm{ev}_{2}^{*} \mathrm{vol}_{L(\mathbf{u})}$ extends to this cobordism. Therefore Lemmas 12.27 and 12.28 imply Theorem 12.22 in case $\mathfrak{b}=b=0$. The general case is similar.

REMARK 12.29. According to E. Getzler, the fact $\mathcal{M}_{(1,1) ; 0}\left(\beta ; \Sigma^{(1)}\right)$ is cobordant to $\mathcal{M}_{(1,1) ; 0}\left(\beta ; \Sigma^{(2)}\right)$ is called the Cardy relation.

REMARK 12.30. A similar trick using the annulus is used in $[\mathbf{A b 2}, \mathbf{B C}]$ for a similar but a slightly different purpose.

\section{Examples 3}

EXAmPle 13.1. We consider the case of $\mathbb{C} P^{n}$ and $\mathfrak{b}=\mathbf{0}$. The moment polytope $P$ is a simplex $\left\{\left(u_{1}, \ldots, u_{n}\right) \mid 0 \leq u_{i}, \sum u_{i} \leq 1\right\}$ and the potential function is

$$
\mathfrak{P O}^{\mathbf{0}}=\sum_{i=1}^{n} y_{i}+T\left(y_{1} \ldots y_{n}\right)^{-1}
$$

The critical points are $\mathfrak{y}^{(k)}=\left(T^{\frac{1}{n+1}} e^{\frac{2 \pi \sqrt{-1} k}{n+1}}, \ldots, T^{\frac{1}{n+1}} e^{\frac{2 \pi \sqrt{-1} k}{n+1}}\right), k=0, \ldots, n$ which are all non-degenerate. The isomorphism $\operatorname{Jac}\left(\mathfrak{P O}^{\mathbf{0}}\right) \otimes_{\Lambda_{0}} \Lambda \cong \prod_{k=0}^{n}$ $\Lambda 1_{\mathfrak{y}^{(k)}}$. is induced by

$$
P \mapsto \sum_{k=0}^{n} P\left(\mathfrak{y}^{(k)}\right) 1_{\mathfrak{y}^{(k)}}
$$

We put $\mathbf{f}_{k}=\pi^{-1}\left(\left\{\left(u_{1}, \ldots, u_{n}\right) \in P \mid u_{i}=0, i=n-k+1, \ldots, n\right\}\right)$ and $\mathbf{p}_{k}=$ $\mathrm{PD}\left(\mathbf{f}_{k}\right)$ the Poincaré dual to $\mathbf{f}_{k}$. We derive

$$
\mathfrak{P O}^{w \mathbf{p}_{1}}(y)=\mathfrak{P O}^{0}(y)+\left(e^{w}-1\right) y_{n}
$$

from Proposition 4.9 [FOOO4] and hence

$$
\mathfrak{k}_{\mathfrak{s}_{0}}\left(\mathbf{p}_{1}\right)=\left[y_{n}\right]=T^{\frac{1}{n+1}} \sum_{k=0}^{n} e^{\frac{2 \pi \sqrt{-1} k}{n+1}} 1_{\mathfrak{y}^{(k)}}
$$

by definition of $\mathfrak{k}_{\mathfrak{s}_{0}}$. Using the fact that $\mathfrak{k}_{\mathfrak{s}_{0}}$ is a ring homomorphism, we have

$$
\mathfrak{k}_{\mathfrak{s}_{0}}\left(\mathbf{p}_{\ell}\right)=T^{\frac{\ell}{n+1}} \sum_{k=0}^{n} e^{\frac{2 \pi \sqrt{-1} k \ell}{n+1}} 1_{\mathfrak{y}^{(k)}} .
$$

Note this holds for $\ell=0$ also since $\mathbf{f}_{0}$ is a unit and $\mathfrak{k}_{\mathfrak{s}_{0}}$ is unital.

The Hessian of $\mathfrak{P O}^{\mathbf{0}}$ is given by

$$
\operatorname{Hess}_{\mathfrak{x}^{(k)}} \mathfrak{P O}^{\mathbf{0}}=\left[T^{\frac{1}{n+1}} \frac{\partial^{2}}{\partial x_{i} \partial x_{j}}\left(e^{x_{1}}+\cdots+e^{x_{n}}+e^{-\left(x_{1}+\cdots+x_{n}\right)}\right)\right]_{i, j=1}^{i, j=n}\left(\mathfrak{x}^{(k)}\right)
$$


with $\mathfrak{x}^{(k)}=\frac{2 \pi \sqrt{-1} k}{n+1}$. Therefore

$$
\operatorname{Hess}_{\mathfrak{x}^{(k)}} \mathfrak{P O}^{\mathbf{0}}=T^{\frac{1}{n+1}} e^{\frac{2 \pi \sqrt{-1} k}{n+1}}\left[\delta_{i j}+1\right]_{i, j=1}^{i, j=n} .
$$

It is easy to see that the determinant of the matrix $\left[\delta_{i j}+1\right]_{i, j=1}^{i, j=n}$ is $n+1$. Therefore the residue pairing is given by

$$
\left\langle 1_{\mathfrak{y}^{(k)}}, 1_{\mathfrak{y}^{\left(k^{\prime}\right)}}\right\rangle_{\mathrm{res}}=T^{-\frac{n}{n+1}} e^{-\frac{2 \pi \sqrt{-1} k n}{n+1}} \frac{\delta_{k k^{\prime}}}{1+n} .
$$

Combining (135) and (136), we obtain

$$
\left\langle\mathfrak{k}_{\mathfrak{s}_{0}}\left(\mathbf{p}_{\ell}\right), \mathfrak{k}_{\mathfrak{s}_{0}}\left(\mathbf{p}_{\ell^{\prime}}\right)\right\rangle_{\mathrm{res}}=\frac{1}{n+1} T^{-\frac{n}{n+1}} \sum_{k=0}^{n} e^{-\frac{2 \pi \sqrt{-1} k n}{n+1}} T^{\frac{\ell+\ell^{\prime}}{n+1}} e^{\frac{2 \pi \sqrt{-1}\left(\ell+\ell^{\prime}\right) k}{n+1}}
$$

It follows that (137) is 0 unless $\ell+\ell^{\prime}=n$ and

$$
\left\langle\mathfrak{k}_{\mathfrak{s}_{0}}\left(\mathbf{p}_{\ell}\right), \mathfrak{k}_{\mathfrak{s}_{0}}\left(\mathbf{p}_{n-\ell}\right)\right\rangle_{\mathrm{res}}=1=\left\langle\mathbf{p}_{\ell}, \mathbf{p}_{n-\ell}\right\rangle_{\mathrm{PD}_{\mathbb{C} P^{n}}} .
$$

Thus Theorem 12.16 holds in this case.

REMARK 13.2. There are various works in the case of $\mathbb{C} P^{n}$. See $[\mathbf{T a}$, Bar, Gro1].

ExAmPLE 13.3. We consider the Hirzebruch surface $F_{2}(\alpha)$. We use the notation of Example 10.1. In this case the full potential function for $\mathfrak{b}=\mathbf{0}$ is calculated in [Aur2], [FOOO5] section 2.13 and [FOOO6] section 5 as follows.

$$
\mathfrak{P O}^{0}=y_{1}+y_{2}+T^{2} y_{1}^{-1} y_{2}^{-2}+T^{1-\alpha}\left(1+T^{2 \alpha}\right) y_{2}^{-1} .
$$

The valuation of the critical points are

$$
\left(\mathfrak{v}_{T}\left(\mathfrak{y}_{1}\right), \mathfrak{v}_{T}\left(\mathfrak{y}_{2}\right)\right)=((1-\alpha) / 2,(1+\alpha) / 2)=\mathbf{u} .
$$

It is the same for 4 critical points. Then using the variables $\bar{y}_{i}=y_{i}^{\mathbf{u}}$ we have

$$
\mathfrak{P O}^{\mathbf{0}}=T^{(1-\alpha) / 2}\left(\bar{y}_{2}+\left(1+T^{2 \alpha}\right) \bar{y}_{2}^{-1}\right)+T^{(1+\alpha) / 2}\left(\bar{y}_{1}+\bar{y}_{1}^{-1} \bar{y}_{2}^{-2}\right) .
$$

(See Example 10.1.) (We remark $\mathfrak{v}_{T}\left(\bar{y}_{i}\right)=0$.) The critical point equation is

$$
\begin{aligned}
& 0=1-\bar{y}_{1}^{-2} \bar{y}_{2}^{-2} . \\
& 0=1-2 T^{\alpha} \bar{y}_{1}^{-1} \bar{y}_{2}^{-3}-\left(1+T^{2 \alpha}\right) \bar{y}_{2}^{-2} .
\end{aligned}
$$

This has 4 solutions.

The Hessian matrix of (139) is

$$
\left[\begin{array}{cc}
T^{(1+\alpha) / 2}\left(\bar{y}_{1}+\bar{y}_{1}^{-1} \bar{y}_{2}^{-2}\right) & 2 T^{(1+\alpha) / 2} \bar{y}_{1}^{-1} \bar{y}_{2}^{-2} \\
2 T^{(1+\alpha) / 2} \bar{y}_{1}^{-1} \bar{y}_{2}^{-2} & T^{(1-\alpha) / 2}\left(\bar{y}_{2}+\left(1+T^{2 \alpha}\right)\left(\bar{y}_{2}^{-1}\right)\right. \\
& +4 T^{(1+\alpha) / 2} \bar{y}_{1}^{-1} \bar{y}_{2}^{-2}
\end{array}\right] .
$$


We can easily calculate the determinants of this matrix at the four solutions of $(140),(141)$. The determinants are $4 T, 4 T,-4 T,-4 T$. (See [FOOO5] section 2.13 for the detail of the calculation.)

The Hirzebruch suface $F_{2}(\alpha)$ is symplectomorphic to $S^{2}(1-\alpha) \times S^{2}(1-$ $\alpha$ ), where $S^{2}(1-\alpha)$ is the sphere $S^{2}$ with total area $1-\alpha$. This fact is proved in [FOOO6] Proposition 5.1.

The quantum cohomology of $S^{2}(1-\alpha) \times S^{2}(1-\alpha)$ is generated by $x, y$ that correspond to the fundamental class of the factors $S^{2}(1-\alpha)$ and $S^{2}(1+\alpha)$ respectively. The fundamental relations among them are

$$
x^{2}=T^{1-\alpha} 1, \quad y^{2}=T^{1+\alpha} 1, \quad x y=y x .
$$

We put

$$
e_{ \pm}=\frac{1}{2} T^{-(1-\alpha) / 2}\left(T^{(1-\alpha) / 2} \pm x\right), \quad f_{ \pm}=\frac{1}{2} T^{-(1+\alpha) / 2}\left(T^{(1+\alpha) / 2} \pm y\right) .
$$

Then $e_{-} f_{-}, e_{-} f_{+}, e_{+} f_{-}, e_{+} f_{+}$are the units of the 4 direct product factors of $Q H\left(S^{2}(1-\alpha) \times S^{2}(1+\alpha) ; \Lambda\right)$. We have

$$
\int_{S^{2}(1-\alpha) \times S^{2}(1+\alpha)} e_{-} f_{-} e_{-} f_{-}=\frac{1}{4 T}
$$

Hence

$$
\left\langle e_{-} f_{-}, e_{-} f_{-}\right\rangle_{\mathrm{PD}_{S^{2}(1-\alpha) \times S^{2}(1+\alpha)}}=\frac{1}{4 T} .
$$

We obtain $-1 / 4 T,-1 / 4 T, 1 / 4 T$ from $e_{-} f_{+}, e_{+} f_{-}, e_{+} f_{+}$in the same way. Thus, Theorem 12.16 holds in this case also.

EXAMPLE 13.4. We take the monotone toric blow up of $\mathbb{C} P^{2}$ at one point, whose moment polytope is $\left\{\left(u_{1}, u_{2}\right) \mid 0 \geq u_{1}, u_{2}, u_{1}+u_{2} \leq 1, u_{1} \leq 2 / 3\right\}$. Its unique monotone fiber is $\mathbf{u}=(1 / 3,1 / 3)$. We put $\bar{y}_{1}=y_{1}^{\mathbf{u}}, \bar{y}_{2}=y_{2}^{\mathbf{u}}$. Then the potential function (for $\mathfrak{b}=\mathbf{0}$ ) is:

$$
\mathfrak{P D ^ { 0 }}=T^{1 / 3}\left(\bar{y}_{1}+\bar{y}_{2}+\left(\bar{y}_{1} \bar{y}_{2}\right)^{-1}+\bar{y}_{1}^{-1}\right) .
$$

The condition for $\left(\bar{y}_{1}, \bar{y}_{2}\right)$ to be critical gives rise to the equation:

$$
1-\bar{y}_{1}^{-2} \bar{y}_{2}^{-1}-\bar{y}_{1}^{-2}=0, \quad 1-\bar{y}_{1} \bar{y}_{2}^{2}=0 .
$$

We put $\bar{y}_{2}=z$. Then $\bar{y}_{1}=1 / z$ and

$$
z^{4}+z^{3}-1=0 .
$$

By Theorem 12.13 (3) we have

$$
\begin{aligned}
Z\left(0,\left(\bar{y}_{1}, \bar{y}_{2}\right)\right) & =T^{2 / 3} \operatorname{det}\left[\begin{array}{cc}
\bar{y}_{1}+\left(\bar{y}_{1} \bar{y}_{2}\right)^{-1}+\bar{y}_{1}^{-1} & \left(\bar{y}_{1} \bar{y}_{2}\right)^{-1} \\
\left(\bar{y}_{1} \bar{y}_{2}\right)^{-1} & \bar{y}_{2}+\left(\bar{y}_{1} \bar{y}_{2}\right)^{-1}
\end{array}\right] \\
& =T^{2 / 3} \frac{4-z^{3}}{z} .
\end{aligned}
$$


Let $z_{i}(i=1,2,3,4)$ be the 4 solutions of $(144)$. Then the left hand side of (118) becomes:

$$
T^{-2 / 3} \sum_{i=1}^{4} \frac{z_{i}}{4-z_{i}^{3}} .
$$

We can directly check that $(145)=0$. (See [FOOO5] Example 1.2.36.) Thus we checked that Corollary 12.21 holds in this case.

\section{Acknowledgements}

KF is supported partially by JSPS Grant-in-Aid for Scientific Research No. 18104001 and Global COE Program G08, YO by US NSF grant \# 0904197, HO by JSPS Grant-in-Aid for Scientific Research No. 19340017 and 23340015, KO by JSPS Grant-in-Aid for Scientific Research No. 21244002.

\section{References}

[Ab1] M. Abouzaid, Morse homology, tropical geometry, and homological mirror symmetry for toric varieties, Geom. Topol. 10 (2006), 1097-1157.

[Ab2] M. Abouzaid, A geometric criterion for generating the Fukaya category, Publ. Math. Inst. Hautes Études Sci. No. 112 (2010), 191-240, arXiv:1001.4593.

[AKO] D. Auroux, L. Katzarkov and D. Orlov, Mirror symmetry for weighted projective planes and their noncommutative deformations, Ann. of Math. (2) 167 (2008), no. 3, 867-943, mathAG/0404281.

[Aur1] D. Auroux, Mirror symmetry and T-duality in the complement of an anticanonical divisor, J. Gökova Geom. Topol. 1 (2007), 51-91, arXiv:0706.3207v2.

[Aur2] D. Auroux, Special Lagrangian fibrations, wall-crossing, and mirror symmetry, Surveys in Differential Geometry 13 (2009) 1-47, arXiv:0902.1595.

[Bar] S. Barannikov, Semi-infinite Hodge structures and mirror symmetry for projective spaces, preprint, arXiv:math/0010157.

[B1] V. Batyrev, Quamtum cohomology rings of toric manifolds, Journées de Géométrie Algébrique d'Orsay, 1992, Astérisque 218, 9-34.

[B2] V. Batyrev, Dual polyhedra and mirror symmetry for Calabi-Yau hypersurfaces in toric varieties, J. Alge. Geom. 3 (1994), no. 3, 493-535.

[BC] P. Biran and O. Cornea, Quantum structures for Lagrangian submanifolds, preprint, arXiv:0708.4221.

[BGR] S. Bosch, U. Güntzer and R. Remmert, Non-Archimedean Analysis. Asystematic Approach to Rigid Analytic Geometry, Grundlehren der Mathematischen Wissenschaften 261, Springer-Verlag, Berline, 1984.

[CLa] K. Chan, and S.-C. Lau Open Gromov-Witten invariants and superpotentials for semi-Fano toric surfaces, preprint 2010, arXiv:1010.5287.

[CLe] K. Chan and N.C. Leung, Mirror symmetry for toric Fano manifolds via SYZ transformations, Adv. Math. 223 (2010), no. 3, 797-839, arXiv:0801.2830.

[Cho1] C.-H. Cho, Holomorphic discs, spin structures and the Floer cohomology of the Clifford torus, Internat. Math. Res. Notices 35 (2004), 1803-1843.

[Cho2] C.-H. Cho, Products of Floer cohomology of torus fibers in toric Fano manifolds, Commun. Math. Phys. 260 (2005) 613-640, math.SG/0412414.

[Cho3] C.-H. Cho, Non-displaceable Lagrangian submanifolds and Floer cohomology with non-unitary line bundle, J. Geom. Phys. 58 (2008), 213-226. 
[CO] C.-H. Cho and Y.-G. Oh, Floer cohomology and disc instantons of Lagrangian torus fibers in Fano toric manifolds, Asian J. Math. 10 (2006), 773-814.

[Dub] B. Dubrovin, Geometry of 2D topological field theories, Lecture note in Math. 1620 (1996), 120-348.

[E] M. Entov, K-area, Hofer metric and geometry of conjugacy classes in Lie groups, Invent. Math. 146 (2001), 93-141.

[EP1] M. Entov and L. Polterovich, Calabi quasimorphism and quantum homology, Int. Math. Res. Not. 2003, no. 30, 1635-1676.

[EP2] M. Entov and L. Polterovich, Quasi-states and symplectic intersections, Comment. Math. Helv. 81 (2006), 75-99.

[EP3] M. Entov and L. Polterovich, Rigid subsets of symplectic manifolds, Compositio Math. 145 (2009), 773-826.

[FLTZ] B. Fang, C.-C. M. Liu, D. Treumann, and E. Zaslow A categorification of Morelli's theorem and homological mirror symmetry for toric varieties, Invent math (2011) 186:79-114, arXiv:0811.1228v3.

[Fl1] A. Floer, Morse theory for Lagrangian intersections, J. Differ. Geom. 28 (1988), 513-547.

[Fl2] A. Floer, Cup length estimates on Lagrangian intersections. Comm. Pure Appl. Math. 42 (1989), no. 4, 335-356.

[Fu1] K. Fukaya, Floer homology and mirror symmetry II, Minimal Surfaces, Geometric Analysis and Symplectic Geometry (Baltimore, MD, 1999) Adv. Stud. Pure Math. 34, 31-127, 2002.

[Fu2] K. Fukaya, Differentiable operad, Kuranishi correspondence, and Foundation of topological field theories based on pseudo-holomorphic curve, in Arithmetic and Geometry Around Quantization, Ceyhan et. ed. Birkhäuser Prog. Math. 279 (2010) 123-200.

[Fu3] K. Fukaya, Cyclic symmetry and adic convergence in Lagrangian Floer theory, Kyoto J. Math. 50 (2010) 521-590, arXiv:0907.4219.

[Fu4] K. Fukaya, Counting pseudo-holomorphic discs in Calabi-Yau 3 fold, to appear in Tohoku Math. J., arXiv:0908.0148.

[FOOO1] K. Fukaya, Y.-G. Oh, H. Ohta and K. Ono, Lagrangian Intersection Floer theory - Anomaly and Obstructions -, Part I \& II AMS/IP Studies in Advanced Mathematics, vol 46-1, vol 46-2, 2009, Amer. Math. Soc./International Press, MR2553465, MR2548482.

[FOOO2] K. Fukaya, Y.-G. Oh, H. Ohta and K. Ono, Canonical models of filtered $A_{\infty}$ algebras and Morse complexes, In: New Perspectives and Challenges in Symplectic Field Theory, CRM Proceedings and Lecture Notes 49, 201-227, Amer. Math. Soc. 2009.

[FOOO3] K. Fukaya, Y.-G. Oh, H. Ohta and K. Ono, Lagrangian Floer theory on compact toric manifolds I, Duke. Math. J. 151 (2010), 23-174.

[FOOO4] K. Fukaya, Y.-G. Oh, H. Ohta and K. Ono, Lagrangian Floer theory on compact toric manifolds II: bulk deformations, Selecta Math. New Series, 17 no. 3, (2011), 609-711. arXiv:0810.5654.

[FOOO5] K. Fukaya, Y.-G. Oh, H. Ohta and K. Ono, Lagrangian Floer theory and mirror symmetry on compact toric manifolds, preprint, arXiv:1009.1648.

[FOOO6] K. Fukaya, Y.-G. Oh, H. Ohta and K. Ono, Toric degeneration and nondisplaceable Lagrangian tori in $S^{2} \times S^{2}$, to appear in Int. Math. Res. Not., doi:10.1093/imrn/rnr128, arXiv:1002.1666.

[FOOO7] K. Fukaya, Y.-G. Oh, H. Ohta and K. Ono, Spectral invariants with bulk, quasimorphisms and Lagrangian Floer theory, submitted, arXiv:1105. 5123.

[FOOO8] K. Fukaya, Y.-G. Oh, H. Ohta and K. Ono, Anchored Lagrangian submanifolds and their Floer theory, Contemp. Math. 527 (2010) 15-54. 
[FO] K. Fukaya and K. Ono, Arnold conjecture and Gromov-Witten invariant, Topology, 38 (1999), 933-1048.

[Ful] W. Fulton, Introduction to Toric Varieties, Annals of Math. Studies, 131, Princeton University Press, Princeton, 1993.

[Gi1] A. Givental, Homological geometry and mirror symmetry, in Proceeding of the ICM-1994, Zürich, Basel, 1995, 472-480.

[Gi2] A. Givental, A mirror theorem for toric complete intersections, Topological field theory, primitive forms and related topics (Kyoto, 1996), 141-175, Progr. Math., 160, Birkhäuser Boston, MA, 1998.

[GrPa] T. Graber and R. Pandharipande, Localization of virtual classes, Invent. Math. 135 (1999), 487-518.

[Gro1] M. Gross, Mirror symmetry for $\mathbb{P}^{2}$ and tropical geometry, Adv. Math. 224 (2010), no. 1, 169-245, arXiv:0903.1378.

[Gro2] M. Gross, Toric geometry and mirror symmetry, CBMS Regional Conference Series in Mathematics, 114. Published for the Conference Board of the Mathematical Sciences, Washington, DC; by the American Mathematical Society, Providence, RI, 2011.

[GPS] M. Gross, R. Pandharipande, and B. Siebert, The tropical vertex, Duke Math. J. 153 (2010), no. 2, 297-362, arXiv:0902.0779.

[Gu] V. Guillemin, Kähler structures on toric varieties, J. Differ. Geom. 43 (1994), 285-309.

[Ho] K. Hori, Linear models in supersymmetric D-branes, Proceedings of the KIAS conference - Mirror Symmetry and Symplectic Geometry- (Seoul, 2000), eds by K. Fukaya, Y.-G. Oh, K. Ono and G. Tian, World Sci., River Edge, New Jersey, 2001.

[HV] K. Hori and C. Vafa, Mirror symmetry, preprint, 2000, hep-th/0002222.

[MIRROR] K. Hori, S. Katz, A. Klemm, R. Pandharipande, R. Thomas, C. Vafa, R. Vakil and E. Zaslow, Mirror Symmetry, Clay Mathematics Monographs, Amer. Math. Soc., 2003

[Iri1] H. Iritani, Quantum D-modules and generalized mirror transformations, Topology 47 (2008), 225-276.

[Iri2] H. Iritani, An integral structure in quantum cohomology and mirror symmetry for toric orbifolds, Adv. Math. 222 (2009), 1016-1079.

[Iri3] H. Iritani, Real and integral structures in quantum cohomology I: Toric orbifolds, preprint, arXiv:0712.2204.

[Ko] M. Kontsevich, Homological algebra of mirror symmetry, ICM-1994 Proceedings, Zürich, Birkhäuser, 1995.

[KS1] M. Kontsevich and Y. Soibelman, Homological mirror symmetry and torus fibration, in: Symplectic Geometry and Mirror Symmetry, (Seoul, 2000), eds by K. Fukaya, Y.-G. Oh, K. Ono and G. Tian, pp 203-263, World Sci. River Edge, 2001.

[KS2] M. Kontsevich and Y. Soibelman, Affine structures and non-archimedean analytic spaces, in: The Unity of Mathematics (P. Etingof, V. Retakh, I. M. Singer, eds), pp 321-385, Progr. Math. 244, Birkhäuser 2006.

[Mc] D. McDuff, Displacing Lagrangian toric fibers via probes, preprint, arXiv:0904.1686.

[MS] D. McDuff and D. Salamon, Introduction to symplectic topology. Second edition. Oxford Mathematical Monographs. The Clarendon Press, Oxford University Press, New York, 1998.

[MT] D. McDuff and S. Tolman, Topological properties of Hamiltonian circle actions, Int. Math. Res. Pap. 2006, 72826, 1-77.

[NNU1] T. Nishinou, Y. Nohara, and K. Ueda, Toric degenerations of GelfandCetlin systems and potential functions, Adv. Math. 224 (2010), 648-706., arXiv:0810.3470. 
[NNU2] T. Nishinou, Y. Nohara, and K. Ueda, Potential functions via toric degenerations, preprint, arXiv:0812.0066.

[Os] Y. Ostrover, Calabi quasi-morphisms for some non-monotone symplectic manifolds, Algebr. Geom. Topol. 6 (2006), 405-434.

[OT] Y. Ostrover and I. Tyomkin, On the quantum homology algebra of toric Fano manifolds, Selecta Math. 15 (2009), 121-149, arXiv:0804.0270.

[Sab] C. Sabbah, Universal unfolding of Laurent polynomials and $t t^{*}$ structures, in "From Hodge Theory to Integrabiliy and TQFT $t t^{*}$ geometry", Proceeding Symposia in Pure Mathematics 78 (2008) 1-31.

[Sa] K. Saito, Period mapping associated to a primitive form, Publ. R.I.M.S. 19 (1983), 1231-1261.

[SaTa] K. Saito and A. Takahashi, From primitive forms to Frobenius manifolds, in "From Hodge Theory to Integrabiliy and TQFT $t t^{*}$ geometry", Proceeding Symposia in Pure Mathematics 78 (2008) 31-48.

[MSa] M. Saito, On the structure of Brieskorn lattices, Ann. Institute Fourier (Grenoble) 39 (1989) 27-72.

[Se1] P. Seidel, $\pi_{1}$ of symplectic automorphism groups and invertibles in quantum homology rings, Geom. Funct. Anal. 7 (1997), no. 6, 1046-1095.

[Se2] P. Seidel, More about vanishing cycles and mutation, Symplectic geometry and mirror symmetry (Seoul, 2000), 429-465, World Sci. Publ., River Edge, NJ, 2001.

[Se3] P. Seidel, Symplectic homology as Hochschild homology. Algebraic GeometrySeattle 2005. Part 1, 415-434, Proc. Sympos. Pure Math., 80, Part 1, Amer. Math. Soc., Providence, RI, 2009.

[SYG] A. Strominger, S. Yau and E. Zaslow Mirror symmetry is T-duality. Nucl. Phys. B 479 (1996) 243-259.

[Ta] A. Takahashi, Primitive forms, topological LG model coupled with gravity, and mirror symmetry, preprint, arXiv:9802059.

[Us] M. Usher, Spectral numbers in Floer theories, Compos. Math. 144 (2008), 1581-1592.

[Ue] K. Ueda, Homological mirror symmetry for toric del Pezzo surfaces, Comm. Math. Phys. 264 (2006), 71-85.

[UY] K. Ueda and M. Yamazaki, Homological mirror symmetry for toric orbifolds of toric del Pezzo surfaces, preprint, arXiv:math/0703267.

[W] C. Woodward, Gauged Floer theory of toric moment fibers, Geom. Funct. Anal. 21 (2011), no. 3, 680-749, arXiv:1004.2841.

Department of Mathematics, Kyoto University, Kyoto, Japan

E-mail address: fukaya@math.kyoto-u.ac.jp

Department of Mathematics, University of Wisconsin, Madison, Wi, USA, \& Department of Mathematics, Postech, Pohang, Korea

E-mail address: oh@math.wisc.edu

Graduate School of Mathematics, Nagoya University, Nagoya, Japan, \& Korea Institute for Advanced Study, Seoul, Korea

E-mail address: ohta@math.nagoya-u.ac.jp

Research Institute for Mathematical Sciences, Kyoto Univerisity, Kyoto, Japan, \& Korea Institute for Advanced Study, Seoul, Korea

E-mail address: ono@kurims.kyoto-u.ac.jp 